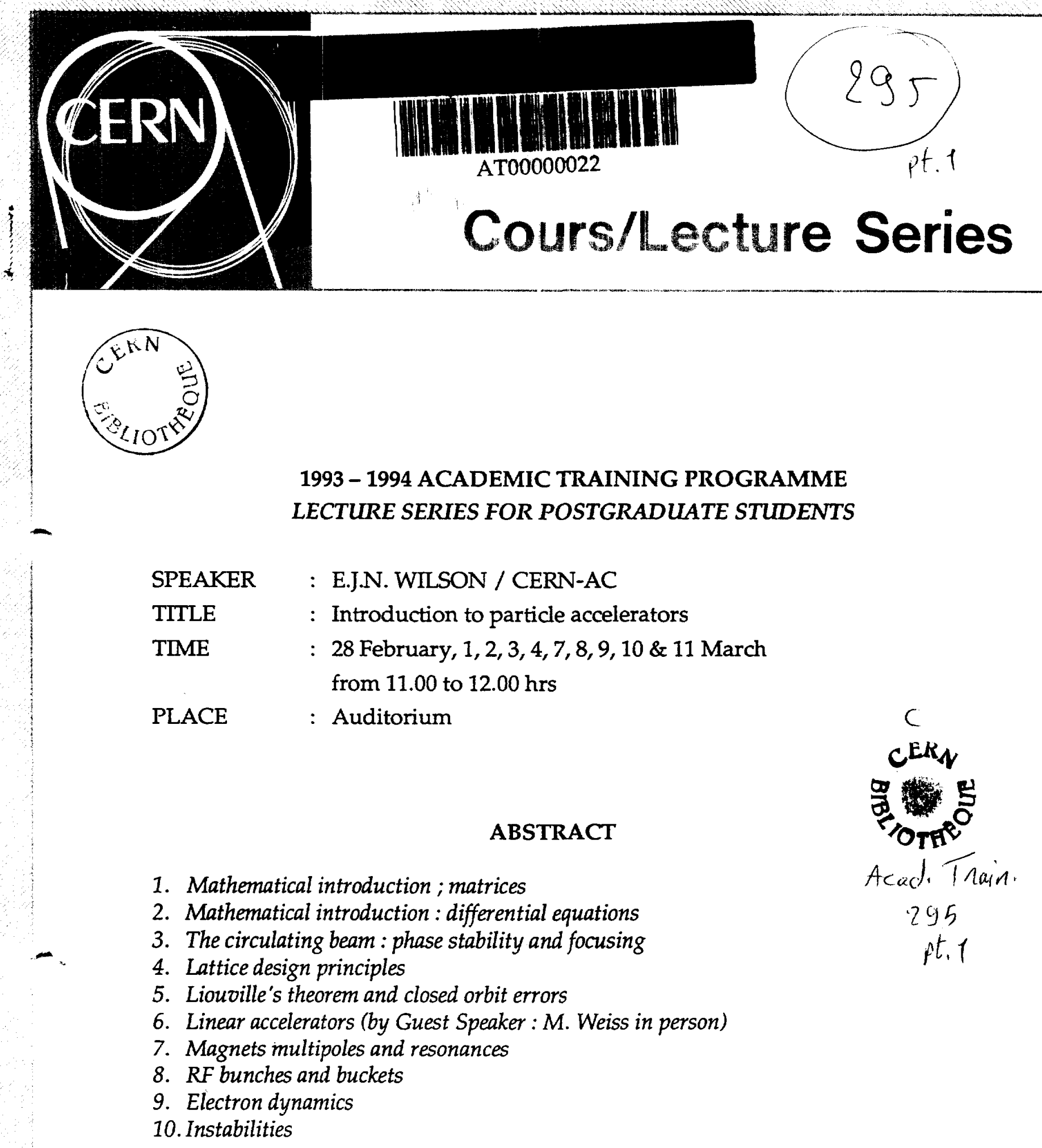

This is a wide-screen video retransmission of a previous popular series.

The course is intended for anyone with a technical or scientific background who would like to become familiar with the principles of accelerator design. It starts with two optional lectures which revise mathematical techniques. The level of the course is chosen 8 with the intention of preparing students for specialist training at the CERN Accelerator School (Level 1) but is also of interest to a wider audience. The ten lectures on all aspects $\vec{x} \dot{x}$ of circular machines cover in a simple way the principles of focusing, betatron motion, \& dispersion, chromaticity, radio-frequency systems, phase stability and beam instabilities. 


\title{
AN INTRODUCTION TO PARTICLE ACCELERATORS
}

\author{
E.J.N.Wilson
}

1. Mathematical Introduction: Matrices

2. Mathematical Introduction: Differential Equations

3. The Circulating Beam : Phase Stability and Focussing

4. Lattices

5. Imperfections and Multipoles

6 Linear Accelerators (by guest speaker M.Weiss)

7. RF Bunches and Buckets

8. Electrons

9. Instabilities

This course is intended for anyone with a technical or scientific background who would like to become familiar with the principles of accelerator design. The level of the course is chosen with the intention of preparing students for the CERN Accelerator School but is also of interest to a wider audience. The nine lectures on all aspects of circular machines were given over the first three months of 1991 and complemented by discussion of set questions in tutorials. They cover in a simple way the principles of focussing, betatron motion, dispersion, chromaticity, radio-frequency systems, phase stability and beam instabilities. Special features of electron machines are treated in one lecture and another, given by guest speaker $\mathrm{M}$. Weiss treats the principles of Linacs 
INTRODUCTION TO PARTICLE ACCELERATORS

LECTURE 1. (MATHS INTRUDUCTION)

"Matrices and Such"

$$
\text { SCALARS }
$$

Multiply: $\quad x=m x \quad($ or $=x m)$

Reciprocal: $x=\frac{1}{m} x \quad$ (or $=m^{-1} x$ )

1.1

1.1 
VECTORS - TwO Numbers

Magnitude and Direction

Eq. (1 $5 \mathrm{~km}$, East (distance), (direction)

(11) Line joining two points
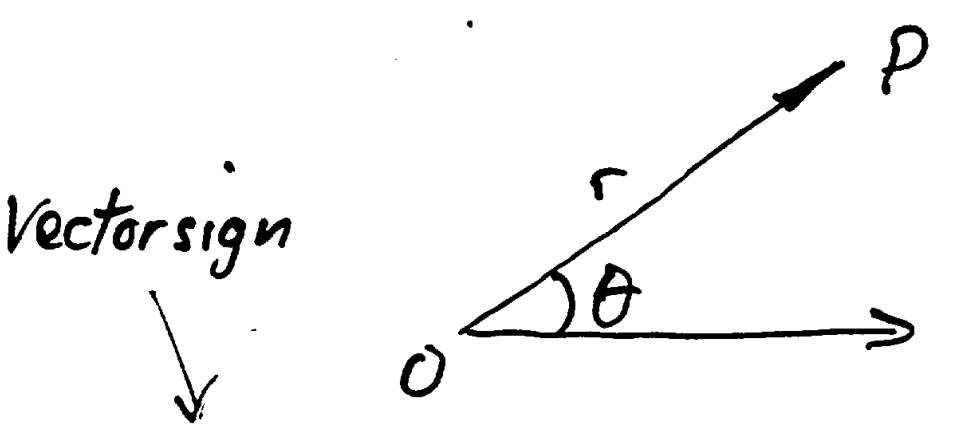

$$
\begin{aligned}
& r=\text { dist } \\
& \theta=\text { direction }
\end{aligned}
$$

Vectorsign<smiles>C1CC1</smiles>

$\bar{r}$ or $(r, \theta)$ or $\overrightarrow{O P}$ or $(x, y)$

1

Polar cartesian.

$x=1 r / \cos \theta$

$$
y=\mid r / \sin \theta
$$

(ii) Velocity

(speed, direction)

$\left(\frac{d s}{d t}, \hat{s}\right)$

unit rector.

$1>$ 
MULTPLYING

Scalar Rule - Doesn't work.

$$
\neq \begin{cases}x=m x & y=m y \equiv \text { Enlarging } \\ R=m r \quad \Theta=m \theta \quad \text { Enlarge+ Rotate }\end{cases}
$$

Vector Rule

In general it po different multipliers four

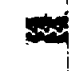
to give nidependent control of $(r, \theta)$ or $(x, y)$

$$
\begin{aligned}
& \gamma_{\text {new }}=\alpha \tilde{r}_{\text {dd }}+\underline{\beta} \theta_{\text {old }} \\
& \theta_{\text {new }}=\underline{\gamma} r_{\text {old }}+\delta \theta_{\text {old }}
\end{aligned}
$$

1.3 
Also in Cartesian.

$$
\begin{aligned}
& x_{\text {new }}=a x_{\text {old }}+b y_{\text {old }} \\
& y_{\text {new }}=c x_{\text {old }}+d y_{\text {old }}
\end{aligned}
$$

IN MATRIX FORM: [just NOTATION]

$$
\left(\begin{array}{l}
x_{n} \\
y_{n}
\end{array}\right)=\left(\begin{array}{ll}
a, & b \\
c & d
\end{array}\right)\left(\begin{array}{l}
x_{\text {old }} \\
y_{\text {old }}
\end{array}\right)_{\text {VECTOR }}
$$

PROVIDED WE OBEY MUILTPLING RULE

[just a technique]

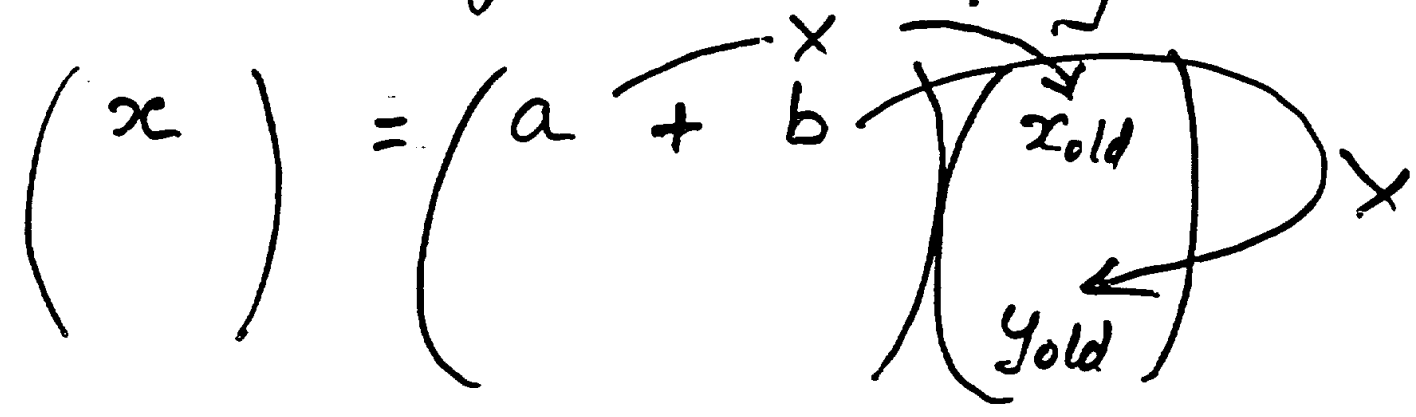

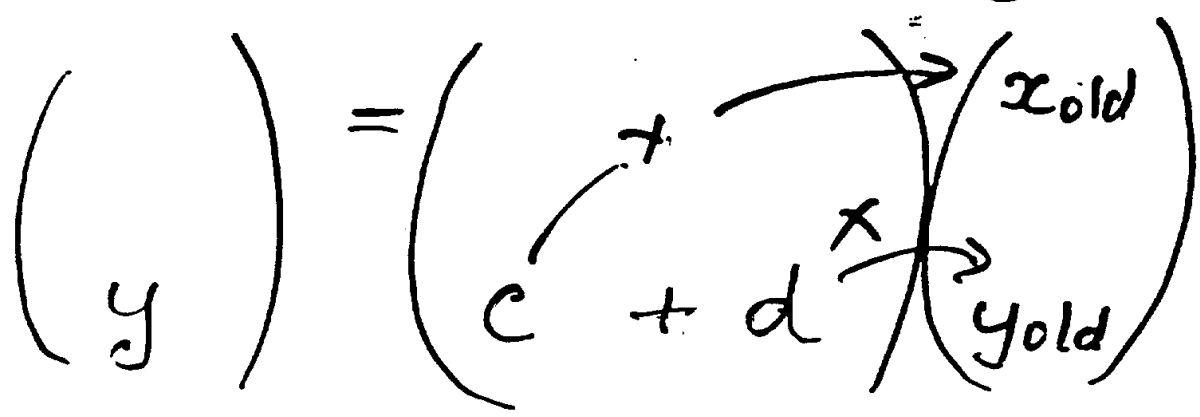

1.1, 
MATRIX OF A XEROX MACHINE

"Copy:"

$$
\begin{aligned}
& \left(\begin{array}{l}
x_{2} \\
y_{2}
\end{array}\right)=\left(\begin{array}{ll}
1 & 0 \\
0 & 1
\end{array}\right)\left(\begin{array}{l}
x_{1} \\
y
\end{array}\right)-x^{*} \\
& x_{2}=x_{1} \quad \text { J also called } \\
& y_{2}=y_{1} \quad \text { unit nation }
\end{aligned}
$$

"Enlarged copy."

$$
\left.\left(\begin{array}{l}
x_{2} \\
y_{2}
\end{array}\right)=\left(\begin{array}{cc}
1.32 & 0 \\
0 & 1.32
\end{array}\right)\left(\begin{array}{l}
x_{1} \\
y_{1}
\end{array}\right) /\right\rangle_{x}
$$

MIRRoR MATRIX

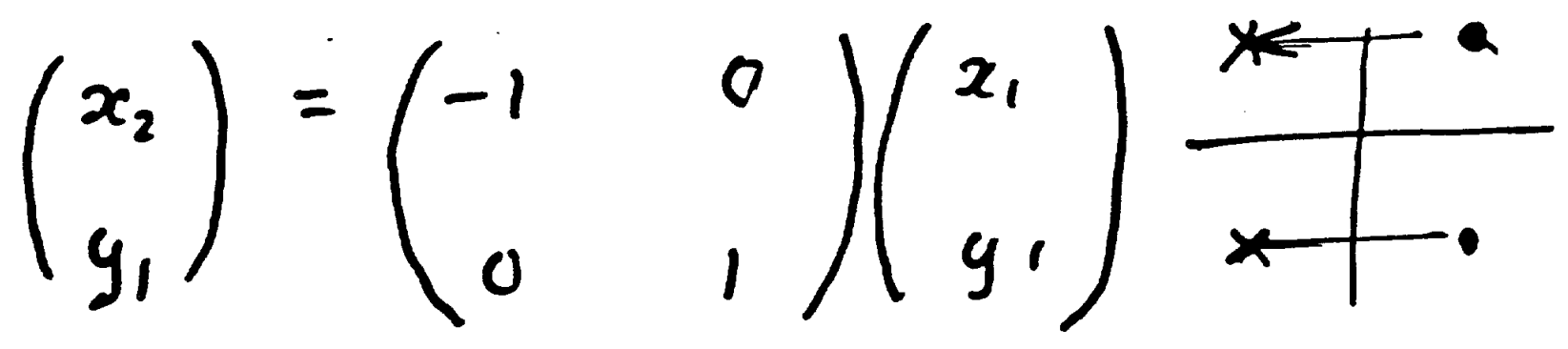

SHEAR MATRIX

$$
\begin{array}{r}
\text { SHEAR MATRIX } \\
x_{2}=x_{1}+b y_{1} \\
y_{2}=y_{1}
\end{array} \quad\left(\begin{array}{l}
x_{2} \\
g_{2}
\end{array}\right)=\left(\begin{array}{ll}
1 & b \\
0 & 1
\end{array}\right)\left(\begin{array}{l}
x_{1} \\
y_{1}
\end{array}\right)
$$


GENERAL RULE

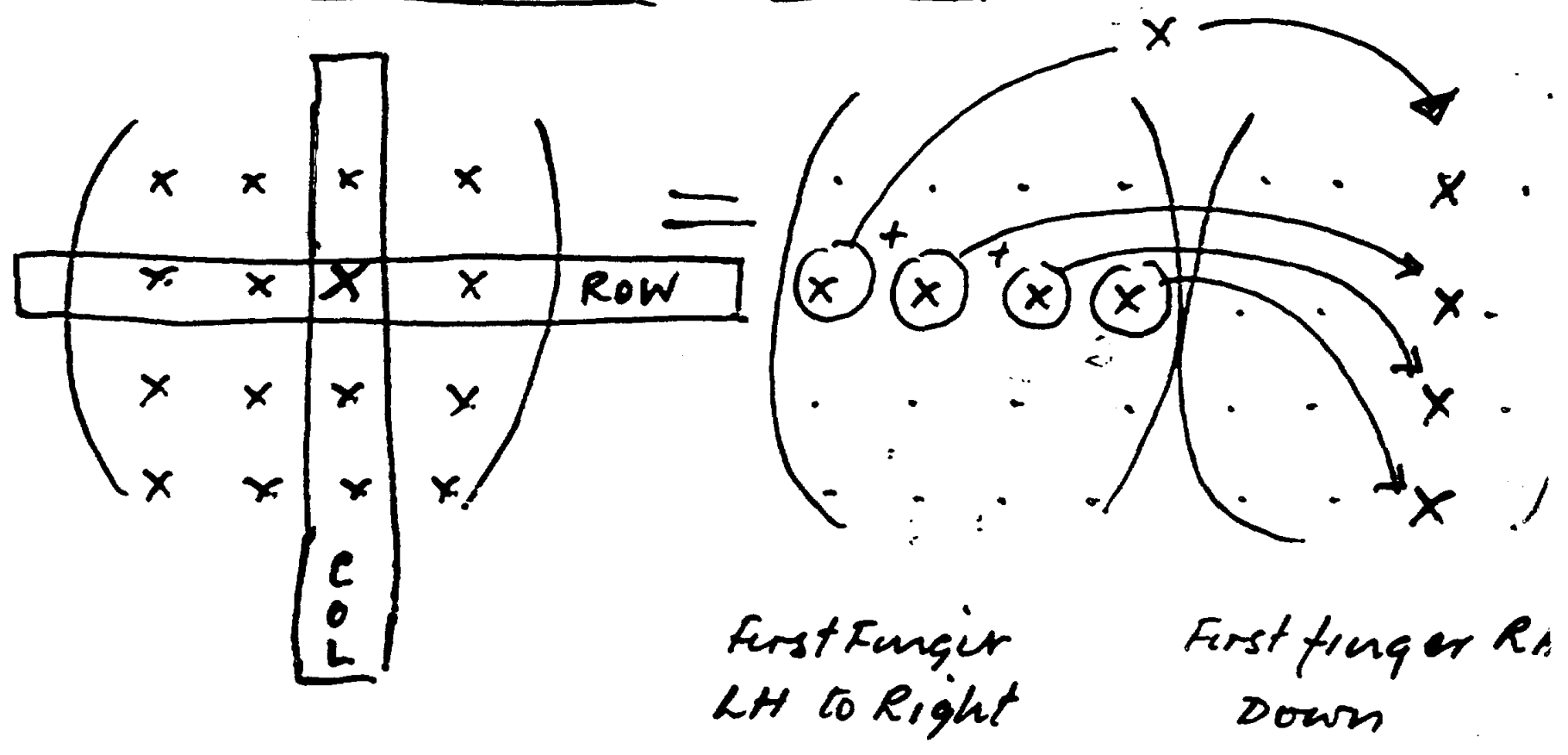

$$
(C)=(B)(A)
$$

$B$ "operates" on $A$ to make $C$

$$
c_{\tau c}=\sum_{i} b_{r i} a_{i c}
$$

! Non Commutative! BA $\neq A B$

IF $D=C B A$

Boperates frost on $A$ Cooperates on (BA) 


$$
\text { TRACE OF A MATRIX }
$$

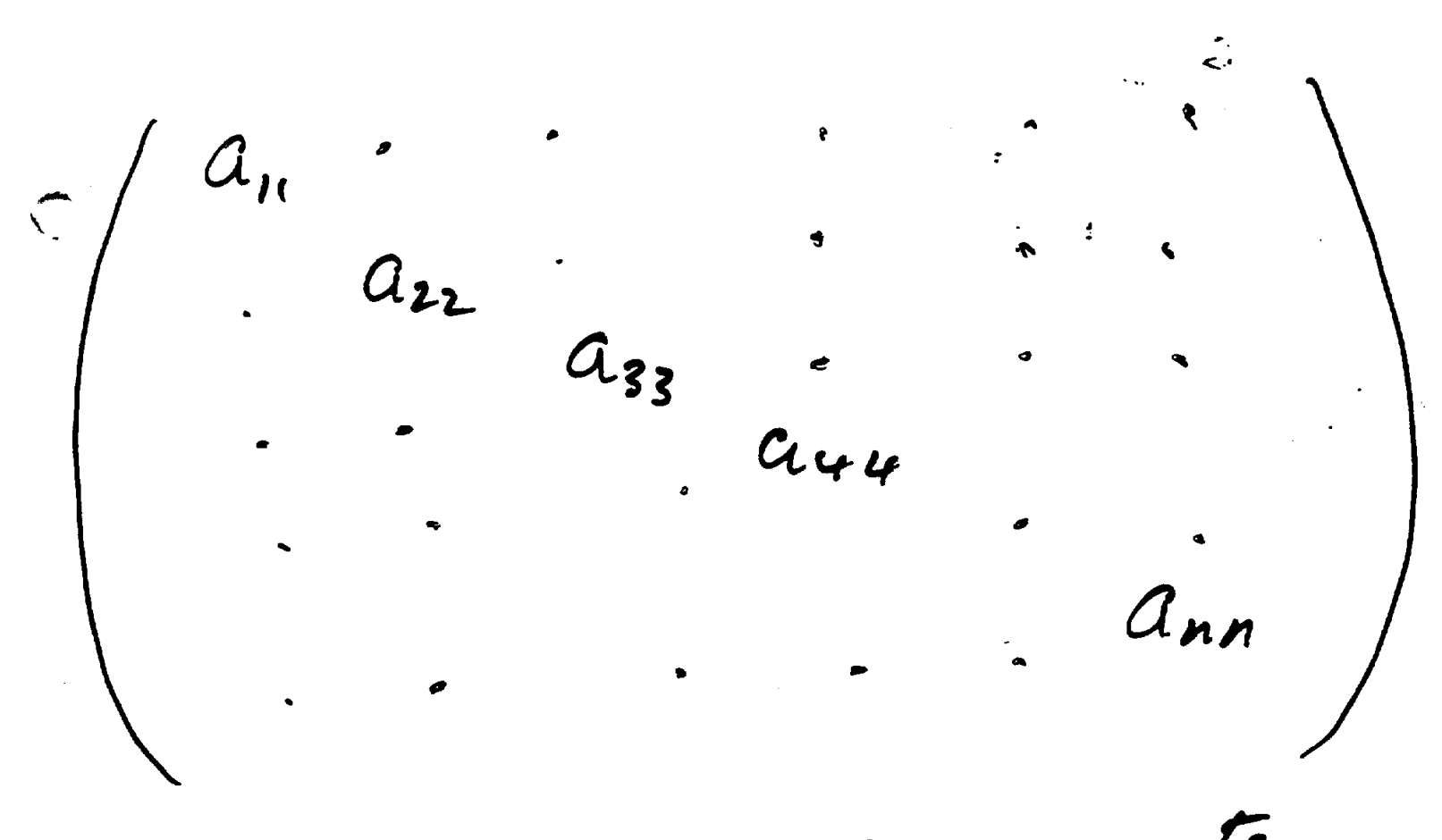

Sum of diagonal elements

$$
\operatorname{Tr}(A)=\sum_{i} a_{i i}
$$

- Useful check on stability of transport matrices $\pi / \pi / 2 \leqslant 1$

- Phase advance $\cos \mu=\pi / 2$

if 
Rows, Curumas, DIAG anal

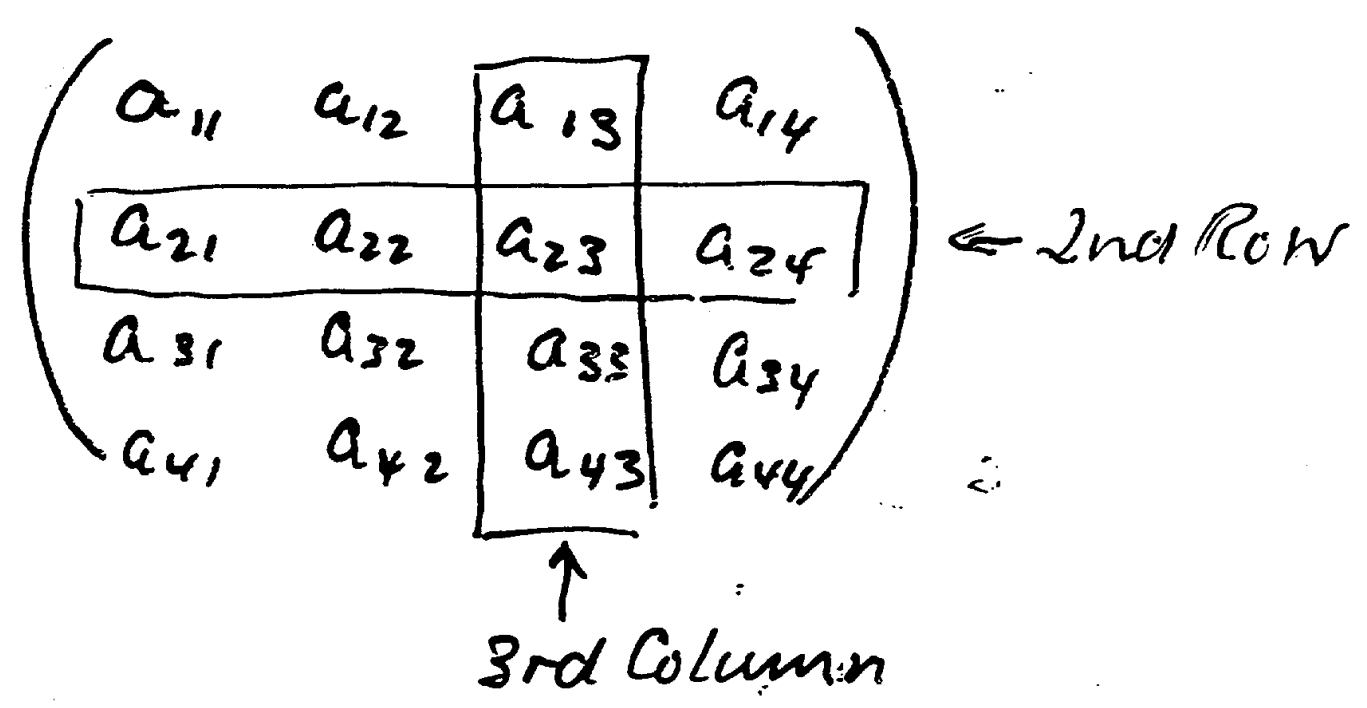

$a_{R C}$ is element

Diagonal Matrix

$$
\left(\begin{array}{l}
x \\
y \\
z
\end{array}\right)=\left(\begin{array}{lll}
a_{11} & 0 & 0 \\
0 & a_{22} & 0 \\
0 & 0 & a_{33}
\end{array}\right)\left(\begin{array}{l}
x \\
y \\
z
\end{array}\right)
$$

- Special property - does not mix $x, y$ and $z$

$-x=a_{11} x, y=a_{22} y, z=a_{33} z$

$x, y, z$ are "CRTHLEGNAL"

$-1.84$ 
DETERMINANTS

$\operatorname{det} M=$ number

For $2 \times 2$ it is easy:

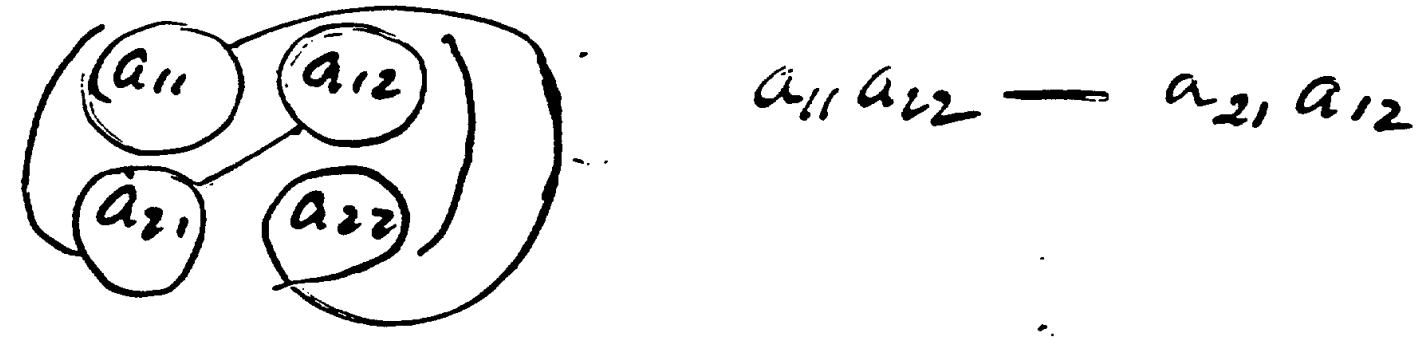

If $\operatorname{det} M=1$ and $M$ is a transformalun -in $x$ and $y$, area is couscrered.

For $3 \times 3$ it is harder

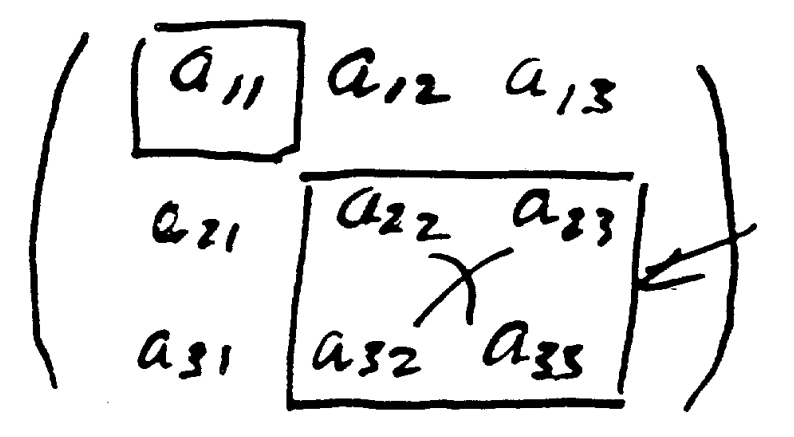

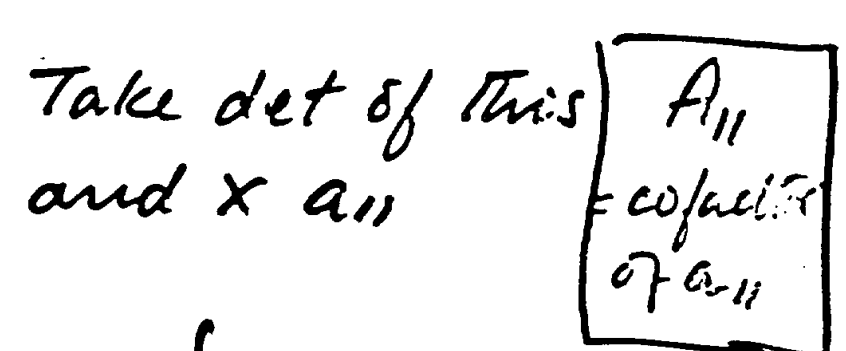

$$
\left(\begin{array}{ccc}
\hat{a}_{11} & a_{12} & a_{13} \\
a_{21} & a_{23} & a_{23} \\
a_{31} & a_{32} & a_{33}
\end{array}\right)
$$

Let of these four and $x(-a, 2)$ all odd terms. (1+2 sid) $t$.

deft of these firs:

$$
\left(\begin{array}{llll}
a_{11} & a_{12} & a_{13} \\
a_{21} X_{22}^{a_{22}} & a_{25} \\
a_{31} & a_{32} & a_{33}
\end{array}\right)
$$
$A$ if cofaction are negative

, 


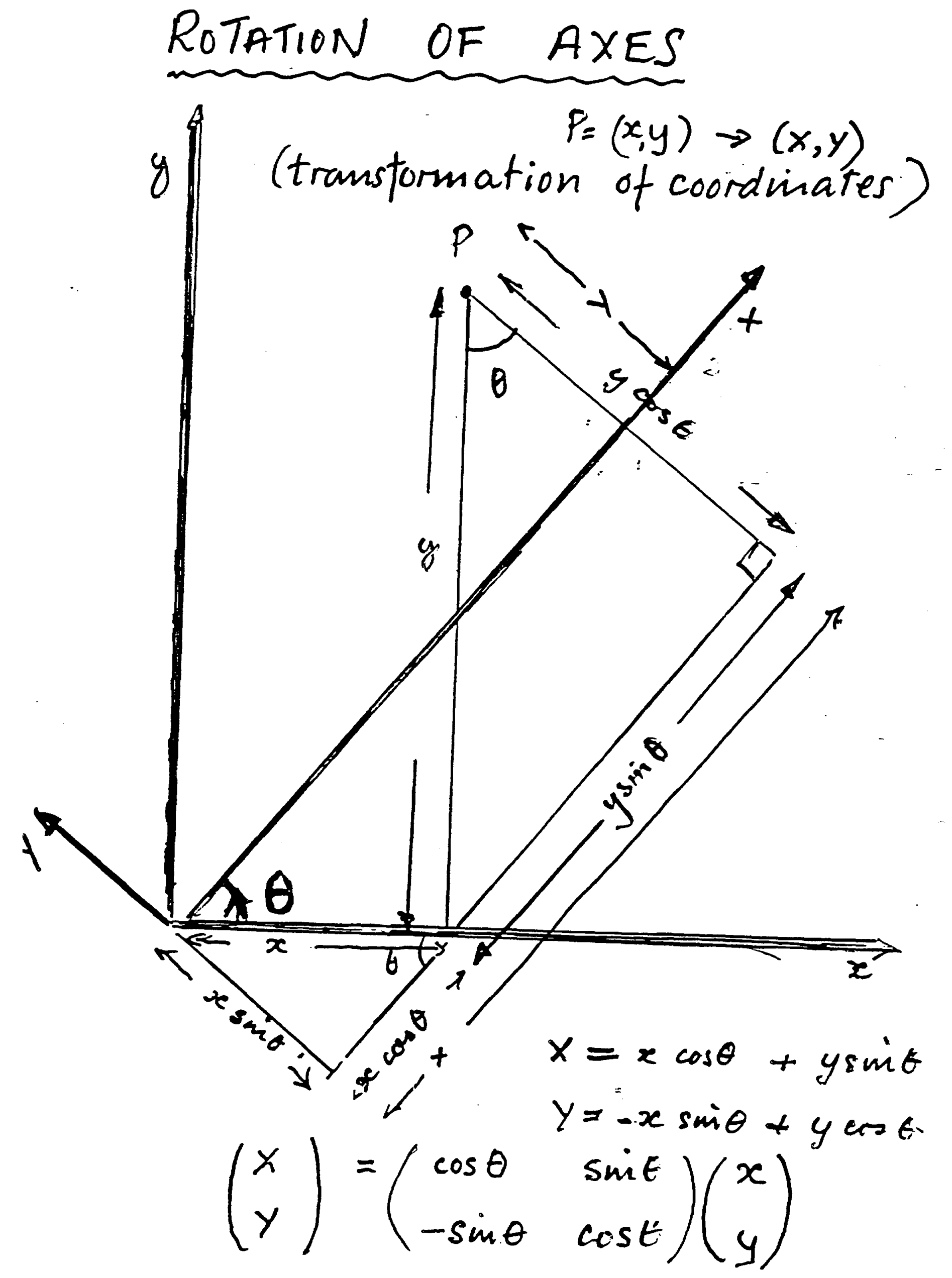


Note suppose we rotate back ide an cungle $\theta$, matrix is then not

$$
R=\left(\begin{array}{c}
\cos \theta, \sin \theta \\
-\sin \theta, \cos \theta
\end{array}\right)
$$

but the reciprocal

$$
\begin{aligned}
R^{-1} & =\left(\begin{array}{cc}
\cos (-\theta), & \sin (-\theta) \\
-\sin (-\theta), & \cos (-\theta)
\end{array}\right) \\
& =\left(\begin{array}{cc}
\cos \theta, & -\sin \theta \\
\sin \theta, & \cos \theta
\end{array}\right)
\end{aligned}
$$

Note also:

$$
\begin{aligned}
\operatorname{det} R & =\cos ^{2} \theta+-\sin ^{2} \theta=1 \\
(R) \cdot\left(R^{-1}\right) & =\left(\begin{array}{c}
\cos ^{2} \theta+\sin ^{2} \theta,-\cos \theta \sin \theta+\cos \theta \sin t \\
-\cos \theta \sin \theta+\sin \theta \cos \theta, \sin ^{2} \theta+\cos ^{2} \theta
\end{array}\right) \\
& =\left(\begin{array}{ll}
1 & 0 \\
0 & 1
\end{array}\right)=\operatorname{CNITMTRR} \times
\end{aligned}
$$


Cofactors of elements

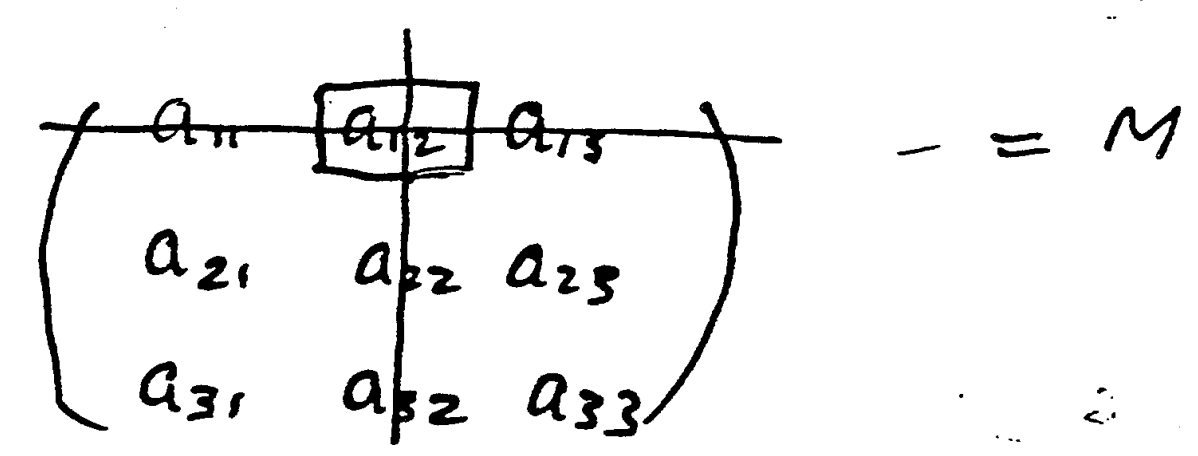

- Look at element ink. (say $a_{12}$ )

- Elements are "odd" if it k =odd (a assad,

- Cofactor is formed by crossing out all elements in same row + colum

TAKE THEIR DETERMINANT AND MULTTPLY BY (-1) if $a_{i K}$ is ODD

Cofactur of $a_{12}$

$$
=A_{12}=(-1)\left(a_{2}, a_{33}-a_{31}, a_{23}\right)
$$

Inverse or RECIPRUCAL MATEIX

This is geist the cofartors/det $M$.

$$
M^{-1}=\frac{1}{\operatorname{det} M}\left(\begin{array}{lll}
A_{11} & A_{21} & A_{31} \\
A_{12} & A_{22} & A_{32} \\
A_{13} & A_{23} & A_{33}
\end{array}\right), \quad M M^{-1}=1
$$

For $2 \times 2$

$$
M^{-1}=\frac{1}{\operatorname{det} \cdot M}\left(\begin{array}{cc}
a_{22}, & -a_{12} \\
-a_{11}, & a_{11}
\end{array}\right)
$$

101.12 
COFACTORS OF ELEMENTS

E

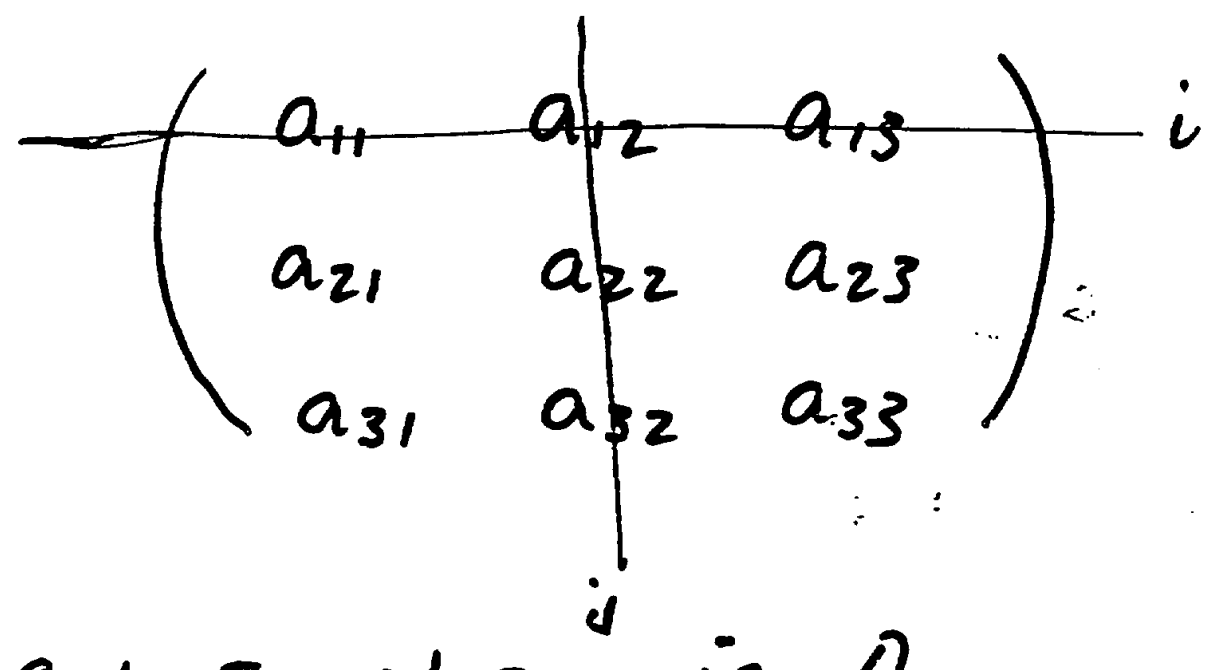

Cofactor of $a_{12}$ is $A_{12}$ " $a_{i j}$ is $A_{i j}$

1. Cross out row $i$ and col j

2. Take DETERMINANT of remainder (called "minor of $a_{i j}$ )

3 Multiply by -1 if $i+j$ is odd ie $x(-1)^{(i+j)}$

GiVING COAACTOR OF $a_{i j}=A_{i d}$

Example $A_{12}=(-1)\left(a_{2}, a_{33}-a_{31}, a_{2.5}\right.$,

1.1310 
INVERSE ON RECIPROCAL MATRIX

$$
M M^{-1}=1 \text { = Unit Matrix }
$$

(eeg. Rotate by an angle then back)

$$
X=M x \text { then } x=M^{-1} x
$$

Lies:- Solves simultaneous equations.

- Reverses paths in a guide field.

$$
M^{-1}=\frac{1}{\operatorname{det} M}\left(\begin{array}{lll}
A_{11} & A_{21} & A_{31} \\
A_{12} & A_{22} & A_{32} \\
A_{13} & A_{23} & A_{33}
\end{array}\right)
$$

Nite A's are cofactors

They have been "Transposed" ice rows $\rightleftharpoons$ columns.

For $2 \times 2$

$$
M=\left(\begin{array}{ll}
a_{11} & a_{12} \\
a_{21} & a_{22}
\end{array}\right) \quad M^{-1}=\frac{1}{\operatorname{det} M}\left(\begin{array}{cc}
a_{22}, & -a_{12} \\
-a_{21}, & a_{11}
\end{array}\right)
$$


Solving Simultaneous Equation's

$Q_{H}$ and $Q_{V}$ are related to Fond $D$ Quadrupole currents, jointly:

$$
\begin{aligned}
L_{Q_{H}} & =a \Delta I_{F}+b \Delta I_{\Delta} \\
\Delta Q_{V} & =c \Delta I_{F}+d \Delta I_{\Delta} \\
\left(\begin{array}{l}
\Delta Q_{H} \\
\Delta Q_{r}
\end{array}\right)= & \left(\begin{array}{ll}
a, & b \\
e, & d
\end{array}\right)\left(\begin{array}{l}
\Delta I_{F} \\
\Delta I_{D}
\end{array}\right)
\end{aligned}
$$

DO AN EXPERIMENT CHANGING IF, ID IN TURN

Measure $\left(\begin{array}{c}\Delta Q_{H} \\ \Delta Q_{V}\end{array}\right)=M\left(\begin{array}{c}\Delta I_{F} \\ \Delta I_{\Delta}\end{array}\right)$

$\therefore$ VERT M TO BE ABLE TO FIND $\triangle I$ ' FOR A DESIRED $\triangle Q$.

$$
\begin{gathered}
I=M^{-1} Q \\
\left(\begin{array}{l}
\Delta I_{F} \\
\Delta I_{D}
\end{array}\right)=M^{-1}\left(\begin{array}{c}
\Delta Q F \\
\Delta Q D
\end{array}\right)
\end{gathered}
$$


HOW TU FIND MATRIX ELEMENTS FROM EXPERIMENTS

IN displacement $x$ divergence angle $\left.x^{\prime}\right\}$ vector

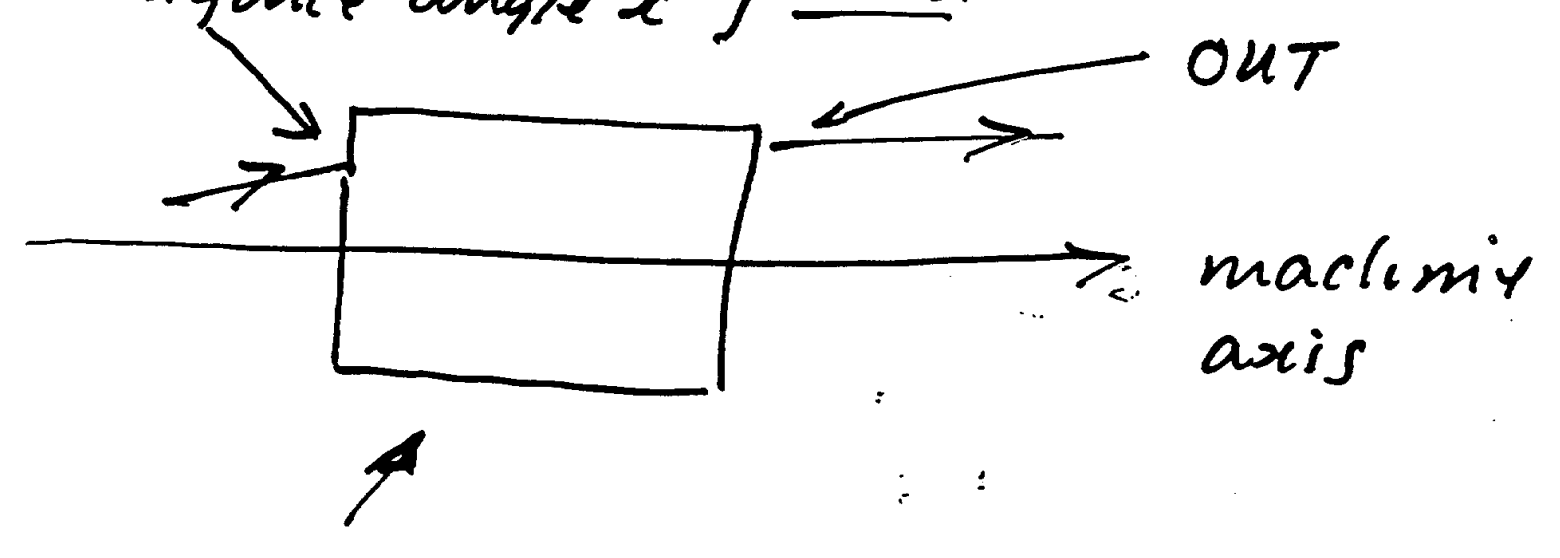

unknown optical system

$$
\left(\begin{array}{c}
x_{\text {out }} \\
x_{\text {out }}^{\prime}
\end{array}\right)=\left(\begin{array}{ll}
a_{11} & \dot{a}_{12} \\
a_{21} & a_{22}
\end{array}\right)\left(\begin{array}{c}
x_{\text {in }} \\
x_{\text {in }}^{\prime}
\end{array}\right)
$$

(1) Try $x_{\text {in }}=1, x_{\dot{m}}^{\prime}=0 \quad$ (cosine $\tilde{c}_{0.1}^{\prime \prime}$

$$
\left(\begin{array}{c}
x_{\text {out }} \\
x_{\text {out }}^{\prime}
\end{array}\right)=\left(\begin{array}{l}
a_{11} \\
a_{21}
\end{array}\right)
$$

sometimes ( $C$ written ( $\left.C^{\prime}\right)$

(2) Try $x_{m i}=0, x_{m}^{\prime}=1$ ("sine cast.

$$
\left(\begin{array}{l}
x_{\text {out }} \\
x^{\prime} \text { out }
\end{array}\right)=\left(\begin{array}{l}
a_{12} \\
a_{22}
\end{array}\right)=\text { souctims }\left(\begin{array}{l}
S^{\prime} \\
\text { written }
\end{array}\left(S^{\prime}\right)\right.
$$

Now we have $\left(\begin{array}{ll}a_{16} & a_{r 2} \\ a_{21} & a_{22}\end{array}\right)=\left(\begin{array}{cc}c, & s \\ c^{\prime}, & s^{\prime}\end{array}\right)$

1.16 
COMPLEX NUMBERS

$$
z=x+i y
$$

Real part Imaginary Part.

$Z$ is just a rector

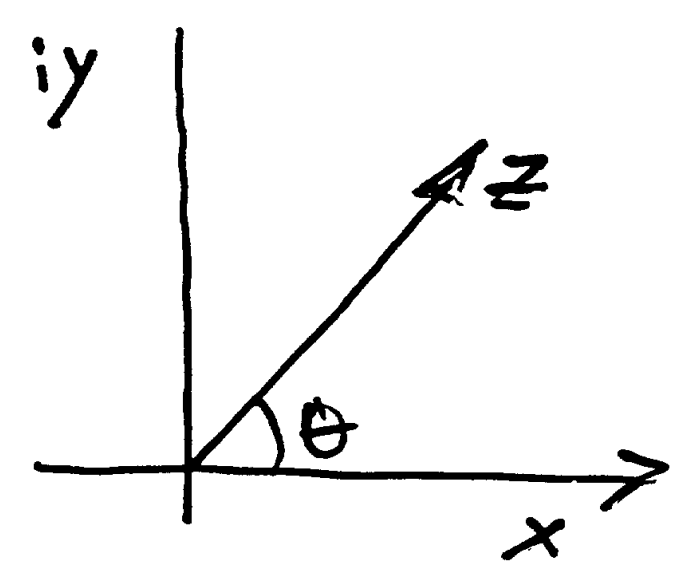

Simple Rules $\quad i=\sqrt{-1}$

$$
\begin{aligned}
& i \times i=-1 \\
& (x+i y)(a+i b)=(a x-b y)+i(a y+b x \\
& (x+i y)^{2}=\left(x^{2}-y^{2}\right)+i(2 x y) \\
& |z|=\left(x^{2}+y^{2}\right)^{1 / 2} i\left[\left[\left(R_{l}\right)^{2}+(I m)^{2}\right]^{1 / 2}\right. \\
& \theta=\tan ^{-1}\left(\frac{y}{x}\right)=\tan ^{-1}\left[\frac{I m}{R_{y}}\right)
\end{aligned}
$$

13 
TO BE COMPLETE

TRANSPOSED ( Conjugate)

$A$ (Nrows, m columns) $\rightarrow A^{\prime}$ (mrows, Neo.

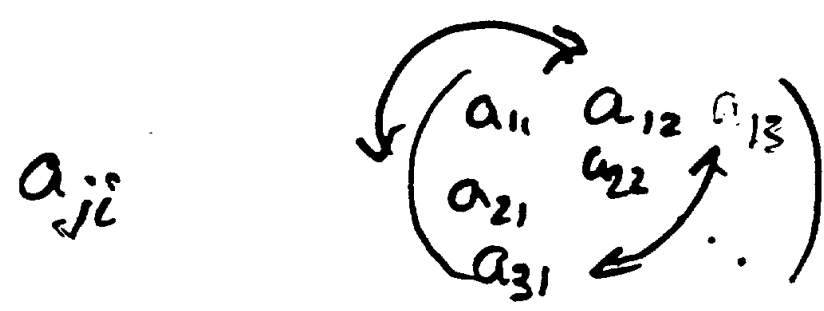

$a^{\prime} i j$

INVERTEA (Reciprocal)

$A$ (square) $\rightarrow: A^{-1}$ (square)

cale cofaclóns $A_{j k}$ and det $\rightarrow a_{j k}^{-\prime}=\frac{A_{k j}}{|A|}$

CRTHOEONAL IF:

$$
A^{\prime}=A^{-1}
$$

HERMITIAN CONJUGATE

$A($ complex,$a+i b) \rightarrow \tilde{A}$

first change $a+i b$ ti $a$-ib then Treansposs

$$
\text { IF } \quad \tilde{A}_{p q}=A_{p q}^{-1}
$$

Maliei is CINITARY OR HERMTTIAN CONITUGAT

(Ased in advanced dynamecs + quanlim? th 
Question Best. Inlloduckion I Parked Acceleration Lect

Q.1. Write down vectors (in cartesian corrdiniater)

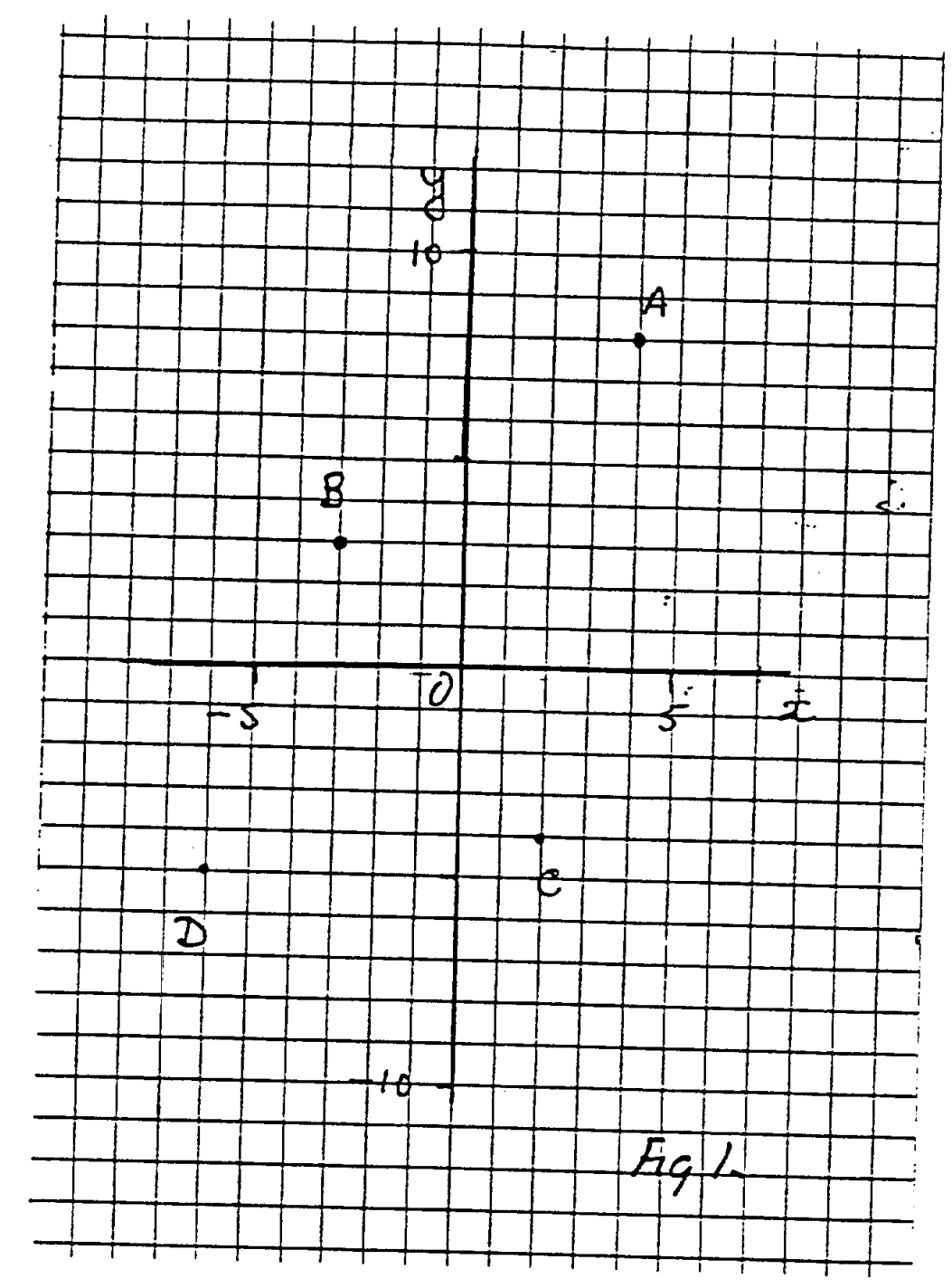

- for each of the points in Fig 1 about from the origin 0

QQ.

$$
\begin{aligned}
& x_{\text {now }}=a x_{\text {old }}+\text { byold } \\
& y_{\text {new }}=c x_{\text {old }}+b \text { yold } \\
& a=1.2 b=-0.5 \quad e=2.32 \quad d=18
\end{aligned}
$$

a)-cakculate $(x, y)$ new for $A B C D$ above.

b) -plot them.

151.16 
Q3.

Fund the prodent of the matrices.

(a) $A=\left(\begin{array}{ll}4 & 2 \\ 1 & 3\end{array}\right)\left(\begin{array}{l}3 \\ 1\end{array}\right)$

(b)

$$
A=\left(\begin{array}{cc}
1, & -4 \\
-2, & 3
\end{array}\right)\left(\begin{array}{l}
7 \\
8
\end{array}\right)
$$

(C)

$$
A=\left(\begin{array}{cc}
m & 0 \\
0 & -m
\end{array}\right)\left(\begin{array}{l}
x \\
y
\end{array}\right)
$$

(d)

$$
A=\left(\begin{array}{cc}
0 & m \\
-m & 0
\end{array}\right)\left(\begin{array}{l}
x \\
y
\end{array}\right)
$$

(e)

$$
A=\left(\begin{array}{ll}
a & b \\
c & d
\end{array}\right)\left(\begin{array}{ll}
\alpha & \beta \\
\gamma & \delta
\end{array}\right)
$$

(f)

$$
A=\left(\begin{array}{ll}
1 & l_{1} \\
0 & 1
\end{array}\right)\left(\begin{array}{ll}
1 & l_{2} \\
0 & 1
\end{array}\right)
$$

(q)

$$
A=\left(\begin{array}{ll}
1 & l_{2} \\
0 & 1
\end{array}\right)\left(\begin{array}{cc}
1 & 0 \\
-\frac{1}{f} & 1
\end{array}\right)\left(\begin{array}{ll}
1 & l_{1} \\
0 & 1
\end{array}\right)
$$

Q4. What is the determineent of the curswer is (E) and $(\varepsilon)$

Le $1: 2$ 
Q5 What is the molisise for:

(a) An elargement by factor $m$.

(6) A rotation by angle $\pi / 4$

(c) A reflection is the-yoy axis

Qb

- the "Q" If a the matins equation relating

$$
\left(\begin{array}{l}
Q_{H} \\
Q_{V}
\end{array}\right)=\left(\begin{array}{cc}
1.2 & 0.3 \\
0.2 & 2.1
\end{array}\right)\left(\begin{array}{c}
I_{F} \\
I_{D}
\end{array}\right)=M / I_{F}
$$

a) What is the determinant $\operatorname{det} / M$ )

b) What ane the coactions of the four elements of $M$ ?

oj What is the reciprocal of $M$. is $M^{-1}$ ?

d) What changes to $I_{F}$ and $I_{D}$ are needed to change $Q_{H}$ by 0.1 mit. 
$A$ A. $A=\left(\begin{array}{l}4 \\ 8\end{array}\right) \quad B=\begin{array}{r}-3 \\ 3\end{array} ; \quad C=\left(\begin{array}{c}2 \\ -4\end{array}\right) \quad D=\left(\begin{array}{c}-6 \\ -5\end{array}\right)$

A 2(a) $\left(\begin{array}{l}0.8 \\ 5.12\end{array}\right) \quad\left(\begin{array}{c}-5.1 \\ 12.36\end{array}\right) \quad\left(\begin{array}{c}4.4 \\ -11.84\end{array}\right) \quad\left(\begin{array}{c}-4.7 \\ 4.92\end{array}\right)$

A3. (a) $\left(\begin{array}{c}14 \\ 6\end{array}\right)$ (b) $\left(\begin{array}{c}-25 \\ 10\end{array}\right) \quad\left(\begin{array}{c}m x \\ -m y\end{array}\right)\left(\begin{array}{c}m y \\ -m x\end{array}\right)$

(e) $\left(\begin{array}{ll}a \alpha+b \gamma, & a \beta+b \delta \\ c \alpha+d \gamma, & c \beta+d \delta\end{array}\right)(f)\left(\begin{array}{cc}1 & 2_{1}+l_{2} \\ 0 & 1\end{array}\right)$

(g) $\left(\begin{array}{cc}1-\frac{l_{2}}{f}, & l_{1}+l_{2}-\frac{l_{1} l_{2}}{f} \\ -\frac{1}{f}, & 1-\frac{l_{1}}{f}\end{array}\right)$

A4. $\quad(a \alpha+b \gamma)(c \beta+\delta d)-(c \alpha+d \gamma)(a \beta+b \delta)$

$a c \alpha \beta+b c \gamma \beta+a d \alpha \delta+b d \gamma \delta$

$-a c \alpha=-a d \beta \gamma-b c \alpha \delta-b d \delta \gamma$

$=a d(\alpha \delta-\beta \gamma)-b c(\alpha \delta-\beta \gamma)=\operatorname{det}\left|\begin{array}{ll}a & b \\ c & d\end{array}\right| \cdot \operatorname{det}\left|\begin{array}{ll}\alpha & \beta \\ \gamma & \gamma\end{array}\right|$

$A_{5}(a)\left(\begin{array}{ll}m & 0 \\ 0 & m\end{array}\right)$

(b) $\left(\begin{array}{cc}\frac{1}{\sqrt{2}}, & \frac{1}{\sqrt{2}} \\ -\frac{-1}{\sqrt{2}} & : \frac{1}{\sqrt{2}}\end{array}\right)$

$(c)\left(\begin{array}{cc}-1 & 0 \\ 0 & 1\end{array}\right)$

$1-22$ 
A6 a) cut/MI $=1.2 \times 2.1-0.2 \times 0.3=2.46$

b)

$\begin{array}{ccc}\text { cofactiv " } & 2.1 \\ 1 & 12 & -0.2 \\ 1 & 21 & -0.3 \\ \because & 22 & 1.2\end{array}$

-

c)

$$
\begin{aligned}
& \frac{1}{2.46}\left(\begin{array}{cc}
1.2 & -0.3 \\
-0.2 & 2.1
\end{array}\right) \\
& =\left(\begin{array}{cc}
0.49 & -0.122 \\
-0.0813 & 0.853
\end{array}\right)
\end{aligned}
$$

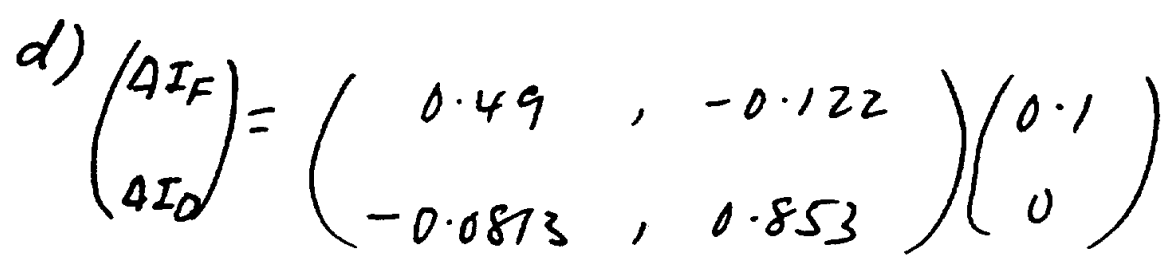

$$
\begin{aligned}
\Delta F_{F} \Delta T_{E} & =0.049 \\
A I_{D} & =-0.00813
\end{aligned}
$$

123 


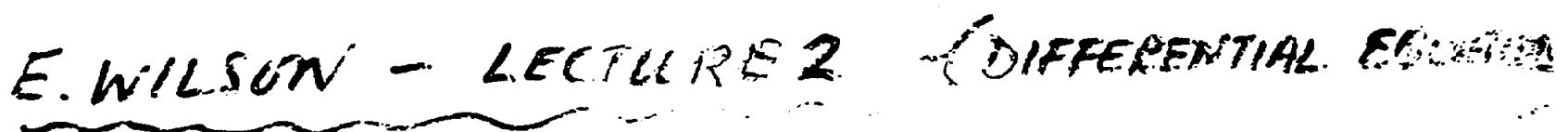
SIMPLE PENDULUM

$$
\begin{aligned}
& l=\text { lengm } \\
& m=\text { mass. }
\end{aligned}
$$

Restoring force

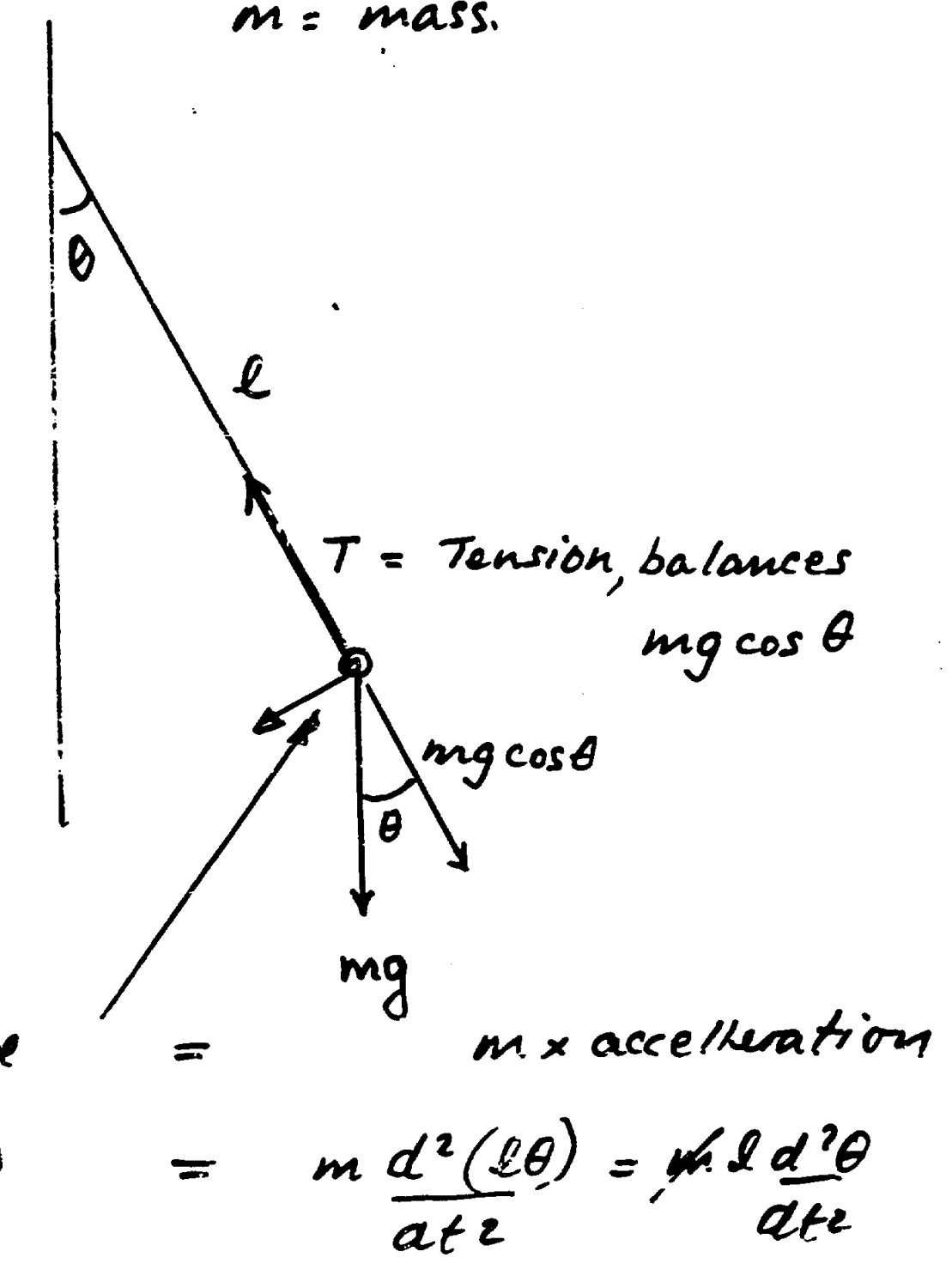

We usually rearrange it and write:

$$
\frac{d^{2} \theta}{d t}+\left(\frac{g}{e}\right) \sin \theta=0
$$

$2 \cdot x^{\prime}$ 
Approximate for $\theta \ll \pi / 2$

$$
\begin{gathered}
\sin \theta=\theta \\
\therefore \quad \frac{d^{2} \theta}{d t^{2}}+\left(\frac{g}{g}\right) \theta=0
\end{gathered}
$$

Note: to save ink we often wile.

$$
\frac{d \theta}{d t}=\dot{\theta} \quad \frac{d^{2} \theta}{d t^{2}}=\ddot{\theta}
$$

So

$$
\ddot{\theta}+\left(\frac{g}{l}\right) \theta=0
$$

is a shorthand way to write it

Note also:

Shorthand for

$$
\frac{d y}{d x}=y^{\prime} \text { and } \frac{d^{\prime} y}{d x^{2}}=y^{\prime \prime}
$$

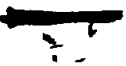

2. 2 
Returning to the pendulum.

$$
\frac{d^{2} \theta}{d t^{2}}+\left(\frac{g}{l}\right) \theta=0 \quad \text { (DIFF. Eu) }
$$

IS a SECOND ORDER DIFFERFNTAL EQUATIN whose coefficients are constant.

We expect (for a pendulums) That the solution will oscillate cion sin aft. or cosizaft where $f$ is the frequency.

Note:

we shall writs

$$
\omega=2 \pi f
$$

in future.

Solving the equation is like nitegraturg twice later will be two arbitrary constants which we choose to be $A=$ amplitude and $\phi=$ starling angle of phase (not slanting $\theta$ )

SOLUTION IS :

$$
\theta=A \cos (\omega t+\phi)-\text { (GuESS) }
$$

BuT WHAT IS W ? 3 
One way to solve the equation and find the value of $\omega$ is gist $t_{0}$ forget the arbitrary constants and substitute our Guess. in the DIFFERENTIAL equation.

$-$

$$
\begin{aligned}
\theta & =A \cos (\omega t+\phi) \\
\frac{d \theta}{d t} & =\dot{\theta}=-\omega A \sin (\omega t+\phi) \\
\frac{d^{2} \theta}{d t^{2}} & =\ddot{\theta}=-\omega^{2} A \cos (\omega t+\phi) \\
\therefore \quad-\omega^{2} A \cos (\omega t+\phi) & +\left(\frac{g}{e}\right) A \cos (\theta+\phi)=0 \\
\omega & =\sqrt{\frac{g}{l}}
\end{aligned}
$$

WE. CAN NOW WRIT DOWN OUR SOLUTION

$$
\theta=A \cos \left(\sqrt{\frac{g}{e}} t+\phi\right)
$$

2.4 
The sane tech ming could be used to solve the displacement $x$ of a weight on a spring

$$
m \frac{d^{2} x}{d t^{2}}+k x=0
$$

This is just Newtons second law. $k$ is the restoring force per unit displacement.

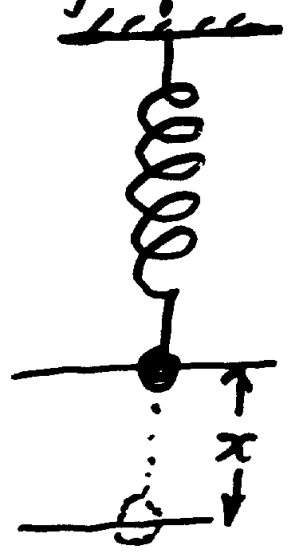

Solution can be found by substituting

$$
x=x_{0} \cos (\omega t+\phi)
$$

amplitude.

Why not try it. "You will find

$$
w=\sqrt{k} m
$$

2.4 
Phase Diagrams

All problems file this will have soluluns

$$
x=x_{0} \cos \left(\omega t+\phi_{0}\right)
$$

Velocity $\dot{x}=\frac{d x}{d t}=-\omega x_{0} \sin (\omega t+\phi)$

Plotting $\dot{x}$ agonist $x$ (two quantities in quadrature makes a circle - or in this case an ellipse)

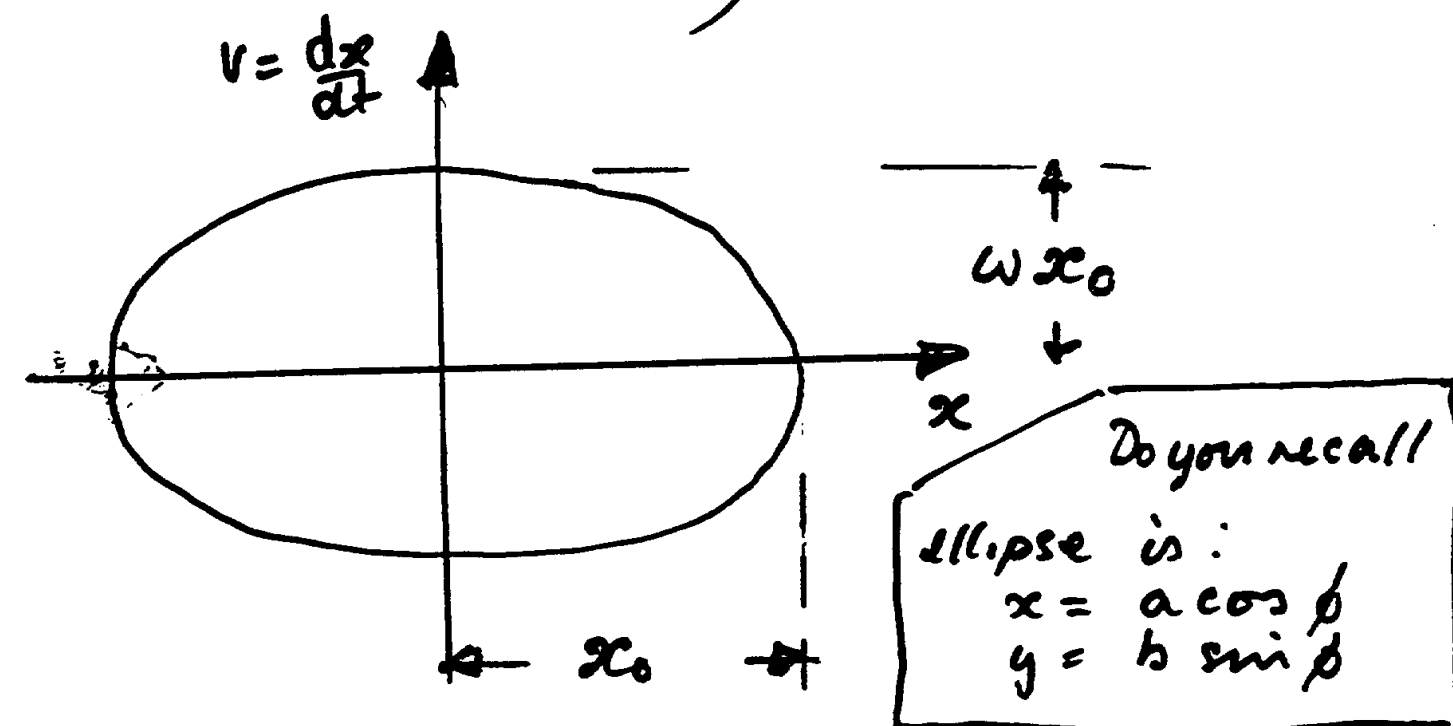

One revolution adds $2 \pi \omega(\omega t+\phi)$ $\phi=(\omega t+\phi)$ is called: The "phase angle" and the diagram is a ph re deagrann" or "phase plot?

IN GENERAL $y$ axis is velocity, momentum! or generous. 
NUMERICAL (COMPUTER) SOLUTION

Suppose we only know the Differential Equation:

$$
m \frac{d^{2} x}{d t^{2}}+k x=0
$$

We can also writs

$$
m \frac{d}{d t}(r)+k x=0
$$

(we could haw? taken a much harder example which cant be soled otherwise is )

This tells us what the change in the "up position in the phase plot is per second when the "left-right" coordinate is $x$.

Taking small intervals of $\Delta t$ we can Face out the phase plot.

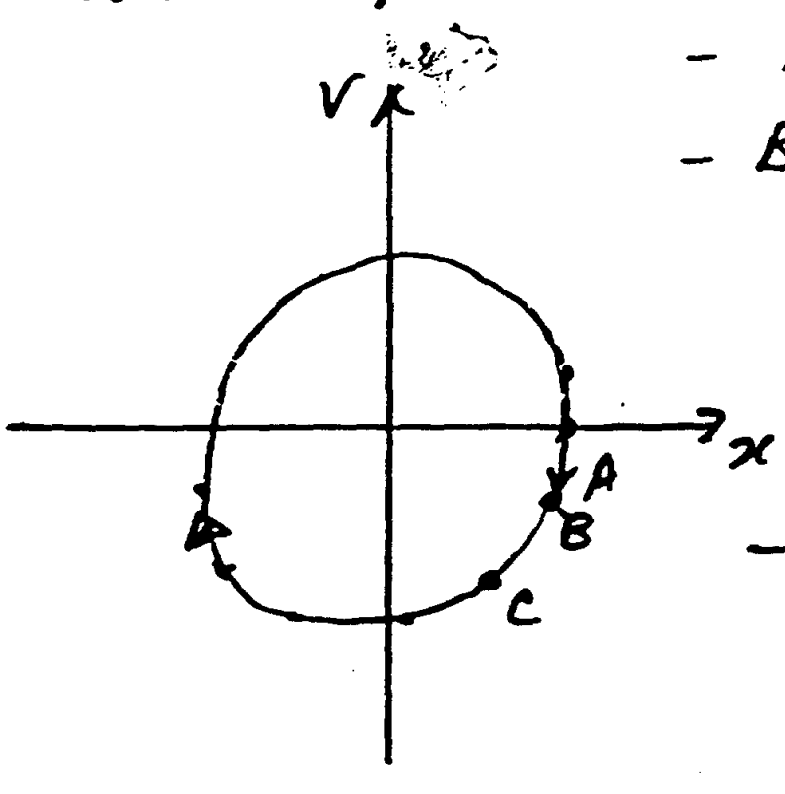

- c $\delta t$ later.

$$
\begin{aligned}
x_{2} & =x_{1}+v \delta t \\
& =x_{1}-\frac{k x_{1}}{m} \delta t^{2} \\
v_{2} & =v_{1}-\frac{k x_{1}}{m} \delta t
\end{aligned}
$$
TRY IT FOR MANY STEPS AND YOU $2 ?$
GET AN ELLIPSE 
RIGID PENDULUM (Example of Numeral

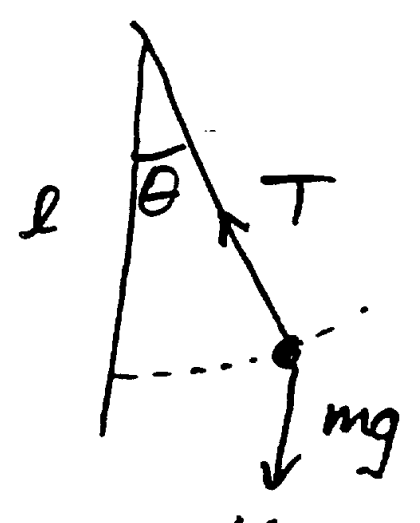
Method)

$$
\begin{aligned}
& m \frac{d^{2}(l \theta)}{d \theta^{2}}+h g \sin \theta=0 \\
& l \frac{d^{2} \theta}{d t^{2}}+g \sin \theta=0
\end{aligned}
$$

Hard because $\theta$ and sm) (not $\theta)$

Put in the new form.

$$
\text { is } \frac{d}{d t}\left(\frac{d \theta}{d t}\right)=-g \sin \theta
$$

(Plot deft or "up" and $\theta$ "loft-regint,

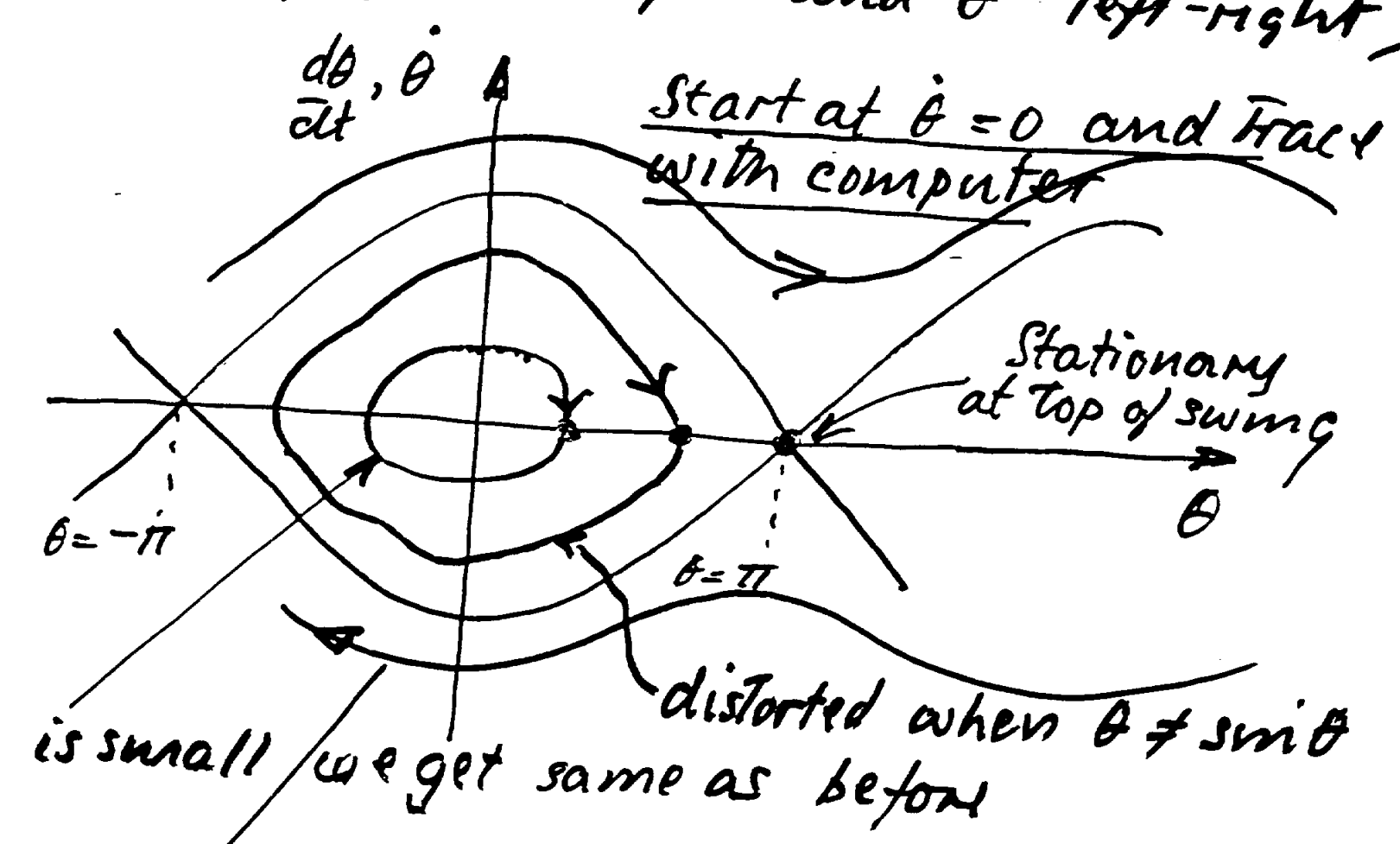

* is small we get same as before

different kid of motion - continuously rotates 
FORCED OSCILLATIONS

$$
m \frac{d^{2} x}{d t^{2}}+k x=f(t)
$$

$f(t)$ can mean exciting the motion of a weight on a spring by attractive it with a solenoid. driven by an oscillator of frequency $w$.

旁

In general, the value of $w$ is not the frequency $\omega_{0}=\sqrt{\mathrm{k} / \mathrm{m}}$ that we get if we solve the motion with $f(t)=0$. $\notin \Phi z_{0} \tau_{0} \cos \omega t$
How much dues the weight mow and with what frequency."

- $\omega_{0}$ or $\omega$ ?

STEP 1 Solve $m \frac{d^{2} x}{d t^{2}}+t x=0$

substitute $\&=a \cos \omega t$

$\operatorname{acos} \omega t\left(m-\omega^{2}-k\right)=0$.

CALLED CHARACTERISTIC EQUATION Gris $\omega_{0}= \pm \sqrt{\mathrm{k} / \mathrm{m}}$ as $1 \mathrm{~s}$ roots.

$2 \cdot 9$ 
SOPHISTICATED DEFINITIONS

$x=a \cos w t$ - our "guessed solution"

mo v - k - "Characteristic Equation"

$x=A \cos \left(\omega_{0} t+\phi_{0}\right)-$ general sols

or $A$, cor wot $+B$, smut "COMPLEMENTARY FUNCTION"

STEP 2 (Take it on trust frow r Piaggio)

IF YOU CAN WRITE:

$$
\left(m \omega_{0}^{2}-k\right) a \cos \omega_{0} t=c \quad\left(\begin{array}{c}
\text { we just } \\
\text { did! }
\end{array}\right)
$$

You CAN ACSO wRITE (for w not wo!)

$\left(m \omega^{2}-k\right)$ aces $\omega i t=d \cos f \omega t$

we wrote 1 it is as drawing $f(t)$ before

Solve $\omega$ find $a$ as a function of $d$ the drowning amplitude

Resulting Response.

$$
a=\frac{d}{m \omega^{2}-k}=\frac{d}{k\left(\omega^{2}-\omega_{0}^{2}\right)}
$$

FULL SoLuTion

$$
\left(\omega_{v}=\sqrt{\mathrm{k}} \mathrm{m}\right)
$$

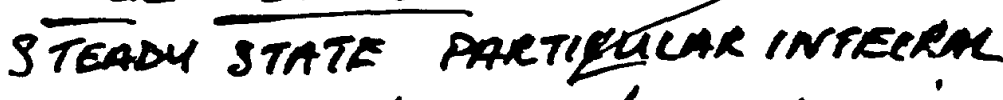

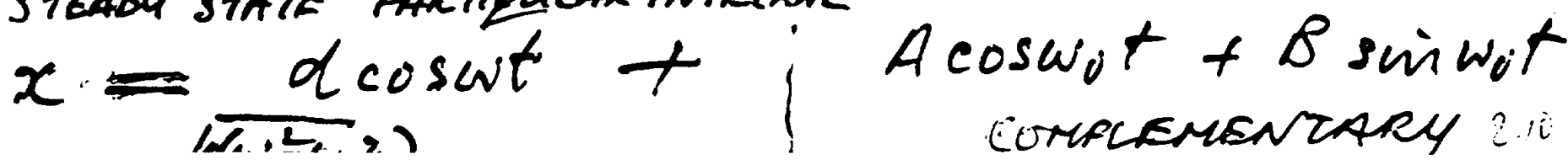


FOURIER SERIES

Any function that repeats every $2 \pi$ in some phase angle can be writes as a harmonic series

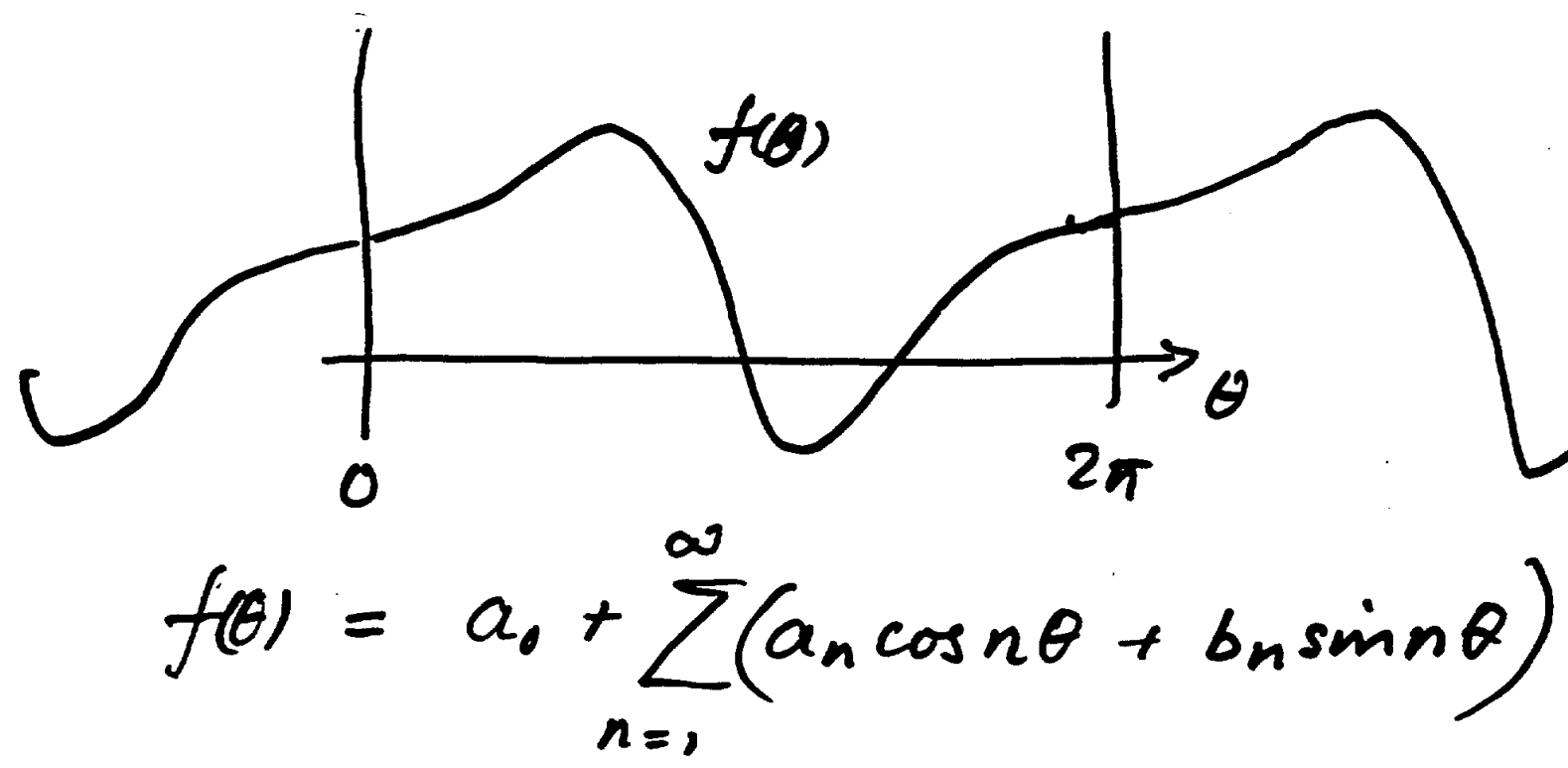

$$
\begin{aligned}
& a_{0}=\text { average value } \frac{1}{2 \pi} \int_{0}^{2 \pi} f(\theta) d \theta . \\
& a_{n}=\int_{0}^{2 \pi} f(\theta) \cdot \cos n \theta d \theta . \\
& b_{n}=\frac{1}{\pi} \int_{0}^{2 \pi} f(\theta) \sin n \theta d \theta
\end{aligned}
$$

PROOF IS TO WRITE $f(\theta)$ IN INTEGRAL AS A SERE

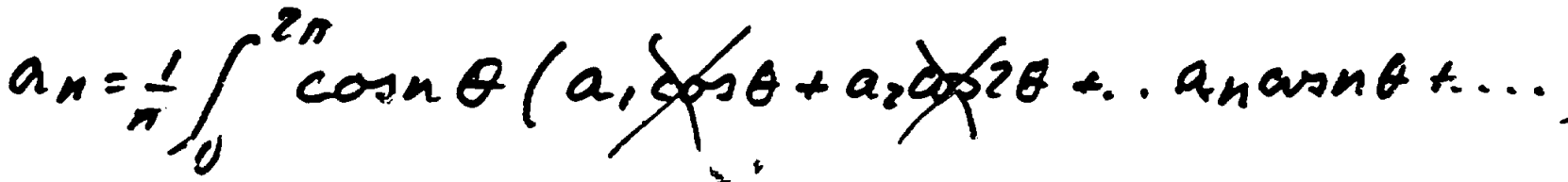

$$
\begin{aligned}
& \int_{0}^{2 \pi} \cos n \theta \cos m \theta=0 \text { if } n \neq 13 \\
& \int_{0}^{2 \pi} d \cos n \theta \cos n \theta=\int_{0}^{\prime \prime} \frac{1}{2}(\cos 2 \theta+1) d \theta=\pi \\
& \therefore n_{n}=a_{n}
\end{aligned}
$$


DIGRESSION ON COMPLEX NUMBERS

THReE ways of writing TheM.

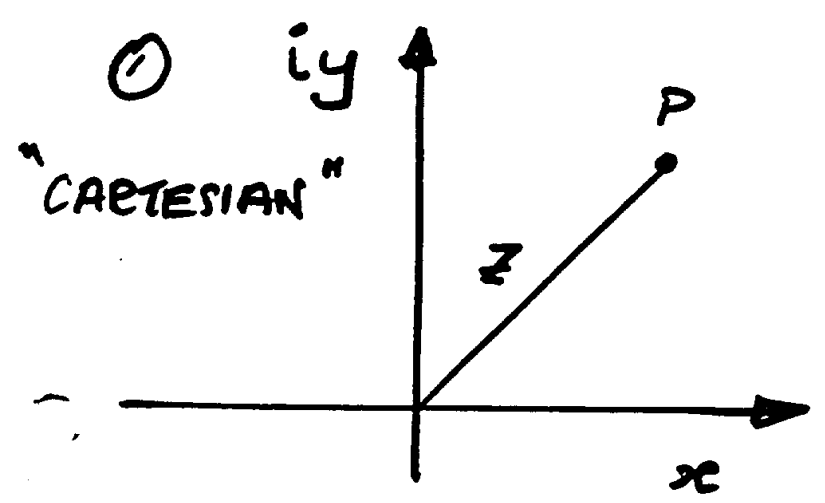

$$
\begin{gathered}
z=x+i y \\
\nearrow \quad \uparrow \\
\text { real pt image part. }
\end{gathered}
$$

(2)

"Polar"

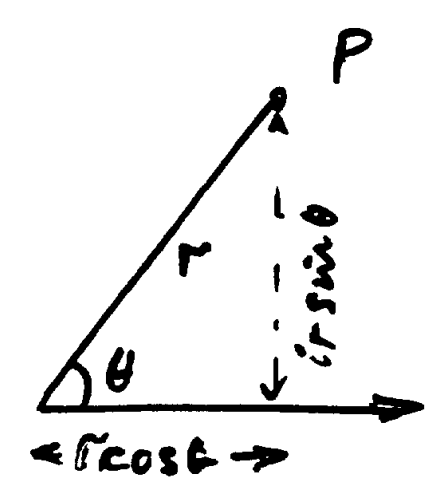

$$
z=r(\cos \theta+i \sin \theta)
$$

(3)

$$
z=A e^{i \theta}
$$

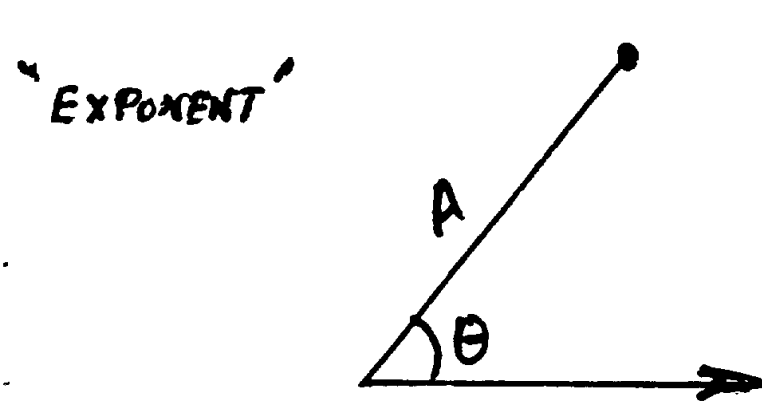

MULTIPLY BY $e^{i \theta}$

$e^{i \theta}$ is defined to mean $(\cos \theta+i \sin \theta)$

$$
\begin{array}{ll}
\ddot{\ddot{\theta}}=0 & e^{i \theta}=1 \\
\theta=\pi / 2 & e^{i \theta}=i \\
\theta=\pi & e^{i \theta}=-1 \\
\theta=3 \pi / 2 & d^{i \theta}=-i
\end{array}
$$

Real

MEANS ROTATE BY ANGLE 6 :

2.2 
IF YOU ARE WORRIED ABOUT $\mathrm{e}^{i E}$ STILL.

Remember: $\cosh x=\left(e^{x}+e^{-x}\right) / 2$

$$
\left.\begin{array}{rl}
\sinh x & =\left(e^{x}-e^{-x}\right) / 2 \\
\cos x & =\left(e^{i x}+e^{-i x}\right) / 2 \\
\sin x & =\left(e^{i x}-e^{-i x}\right) / 2 i
\end{array}\right\} \text { A }
$$

Substitute in $\cos x+i \sin x$ to obtain d ix

You cavemen see what equations $A$ mean graphically on an ARGANs ( $x=$ real, $y$-ring) plot.

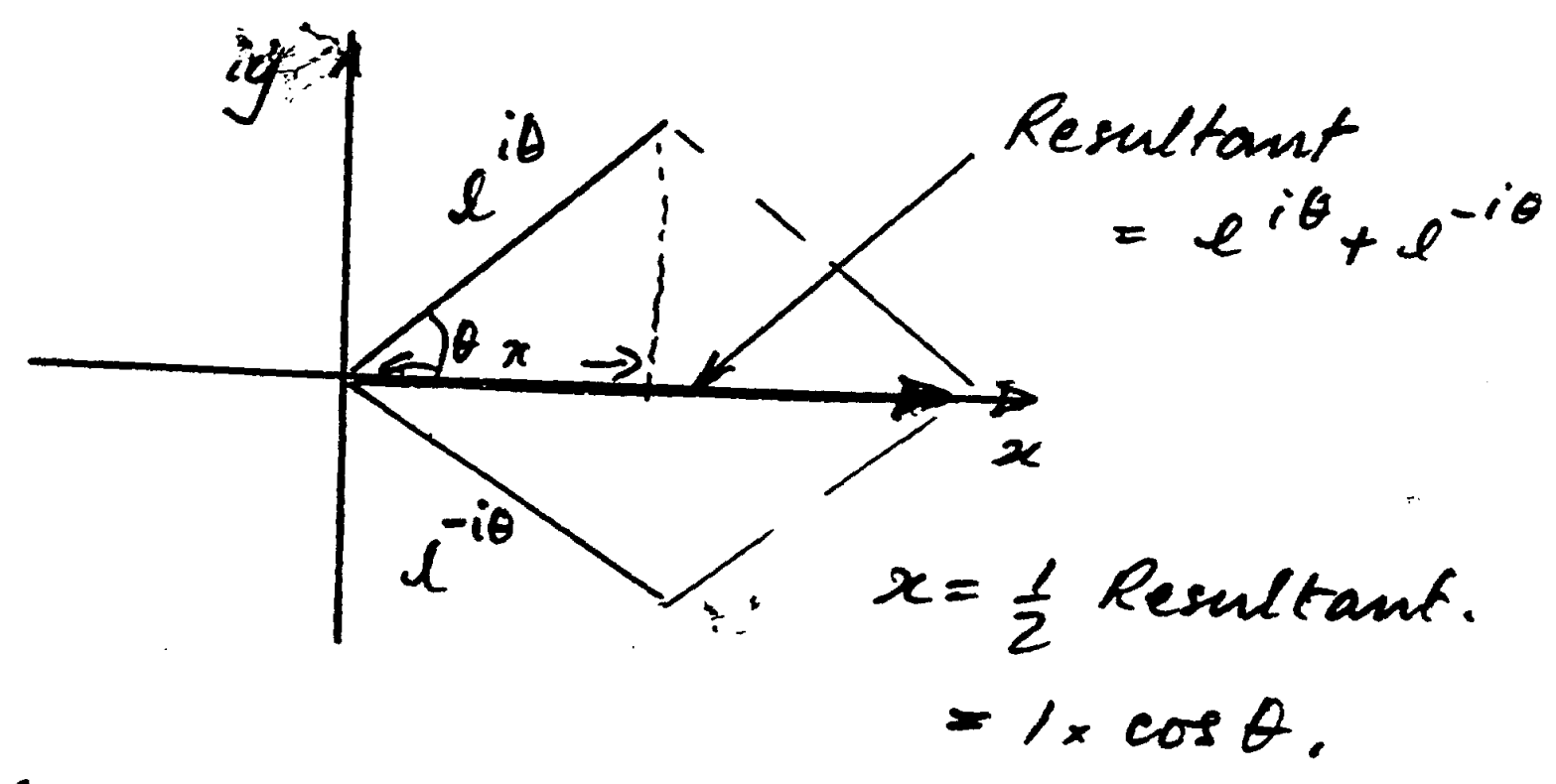

Now TRY to CONSTRUCT $\sin \theta$ !

142.13 
EXPONENT METHOD IS POWERFUL ToOL!

Example:

What is $\sin 2 \theta, \cos 2 \theta$ esconessed as function of $\sin \theta, \cos \theta$ etc.

$$
e^{i 2 \theta}=e^{i \theta} \times e^{i \theta}
$$

(just like ordinary $x^{2}=x \times x$ )

$$
\cos 2 \theta+i \sin 2 \theta=(\cos \theta+i \sin \theta)(\cos \theta+i \sin \theta
$$$$
=\cos ^{2} \theta+2 i \sin \theta \cos \theta-\sin ^{2} \theta
$$

Collecting real terms to the left

$$
=\left(\cos ^{2} \theta+\sin ^{2} \theta\right)+i(2 \sin \theta \cos \theta)
$$

Equating rEAL TERMS.

$$
\cos 2 \theta=\cos ^{2} \theta-\sin ^{2} \theta
$$

EquaTING IMAGINARY RERNS.

$$
\sin 2 \theta=2 \sin \theta \cos \theta \text {. }
$$

$116-$ 
QUESTION 1

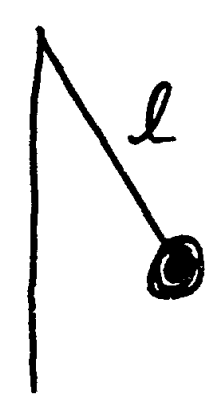

Apendulum has length $l$ and $a$ bob of mass $m$.

a) Show a force diagram

b) What is the restoring force?

c) What is the equation of motion?

d) What is the solution to the equ. of motions.

d) Write down an expression for 101 frequency.

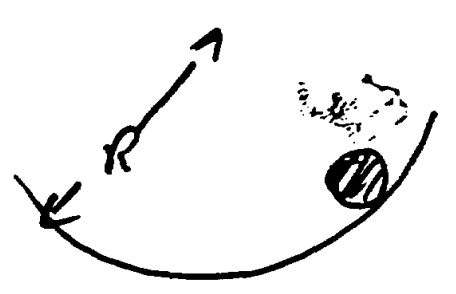

QUESTION 2

A ball rolls down a gutter of radius $R$. Its mass is $m$ and its velocity (out of page) is $r$.

a) Draw a fore e chagrasion to show the restoring force.

b) What is the equation of motion + solute (time is the independent variable)

c) What is the trajectory equation? (sustitula to change $t$ to s)

d) What is the wavelength?

e) If the length of the gutter is $L$ . ...Ln? $/ \angle / a)$ is 
Question 3

a) Given the solution for the motion of the pendulum which starts at maximum suing $(t=0)$ what is the expression for its velocity.

b) What shape is the path traced out by its motion in a phase space chagrin

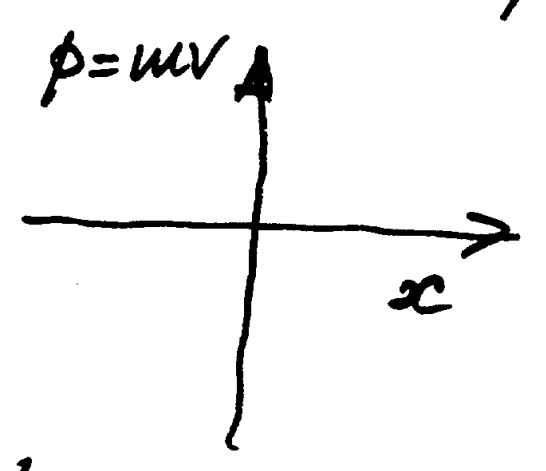

Question 4

Repeationestion 3 for the case of $a$ ball in a gutter.

[Init. The coordinates are

$\left(x, \frac{d x}{d s}\right)$

where $s$ is measured along the gutter .7

$\frac{d x}{d s}$ is also called $x^{\prime}$ or divergence

2.4 
Question 5

Fig. shows the trajectory of a particle in a "drift space"

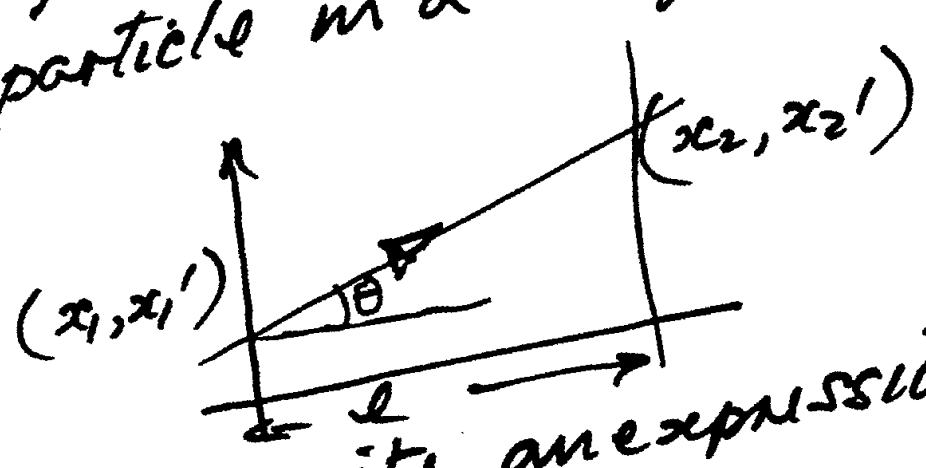

a) write an expression for $\theta$

b) plot a point in phase space $\left(x, x^{\prime}\right)$ corresponding 5 the start and fish.

c) what can we say about $x_{1}^{\prime}$ and $x_{2}^{\prime}$

d) now are $x_{1}$ and $x_{2}$ related to $x^{\prime}$ and $\ell$.

e) Try to write down a matrix with 4 terms which Transforms $\left(x_{3} x_{1}^{\prime}\right)$ into $\left(x_{2}, x_{2}^{\prime}\right)$

(you may leave this until after the next set of questions) 
Question 6

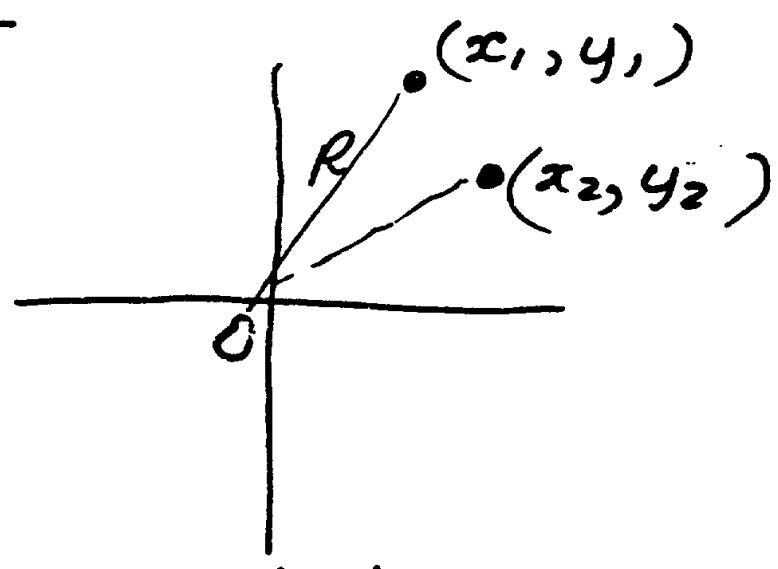

Aponit $\left(x_{1}, y_{1}\right)$ rotates about 0 to $\left(x_{2}, y_{2}\right)$

a) write down equations for

$$
\begin{aligned}
& x_{2}=? x_{1}+? y_{1}^{\prime} \\
& y^{\prime}=? x_{1}+? y_{1}^{\prime}
\end{aligned}
$$

b) Thisis a linear Transformation and can be written in malitis form

$$
\left(\begin{array}{l}
x_{2} \\
x_{2}
\end{array}\right)=\left(\begin{array}{ll}
m_{11} & m_{12} \\
m_{21} & m_{22}
\end{array}\right)\left(\begin{array}{c}
x_{1} \\
1
\end{array}\right)
$$

c) What is the matrix ?

d) What is its determinant?

e) What is its universe

45218 
Question 7.

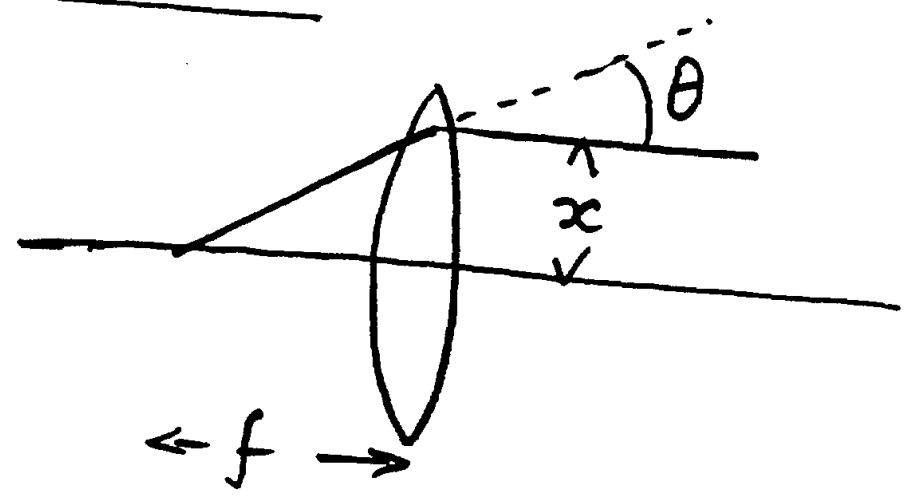

a) There is a change of angle at a convex lens. Write down $\theta$ as a function of $x$.

b) is $\theta$ dependent on where the ray comes from?

c) Can you write down a matrix (2×2) which changes the divergence by 6 but leaves $x$, unchanged.

d) Is this the matrix for a lens? and if so repeat for a divergnig lens.

Questions

$\rightarrow d_{1} \rightarrow 0-\alpha_{2} \rightarrow \nabla$ What are the matrices for this system? What do they do in phase space?

20 
QuEsTION 9.

Any differential equation is suniple to solve if you know the form of the answer.

a)

$$
\begin{aligned}
& \frac{d^{2} x}{d t^{2}}+k x=0 \\
& \frac{d^{2} y}{d s^{2}} \times k y^{2}=0
\end{aligned}
$$

Substitute $x=a e^{ \pm j w t}$ to find $w$ as a function of $t$.

of

b) $\omega$ is not a frequency (the independent variable is s) how is it related to wavelength th

QUESTION NO

- a) Demonstrate that

$$
y=\epsilon^{1 / 2} \beta(\rho)^{1 / 2} \cos (\phi(s)+\lambda)=\epsilon^{1 / 2} \omega(s) \cos (f(s)+\lambda)
$$

is a solvivon of:

$$
\frac{d^{2} y}{d s^{2}}+k(s) y=0
$$

provided $\phi(s)=\int \frac{d s}{\beta(s)}$ and $\omega^{\prime \prime}+k w^{-}-\frac{1}{\omega^{3}}=0$

b) What do you uncienstand by $R(S)$.

$1 x$

$2 \cdot 2$ 
Soln 1.

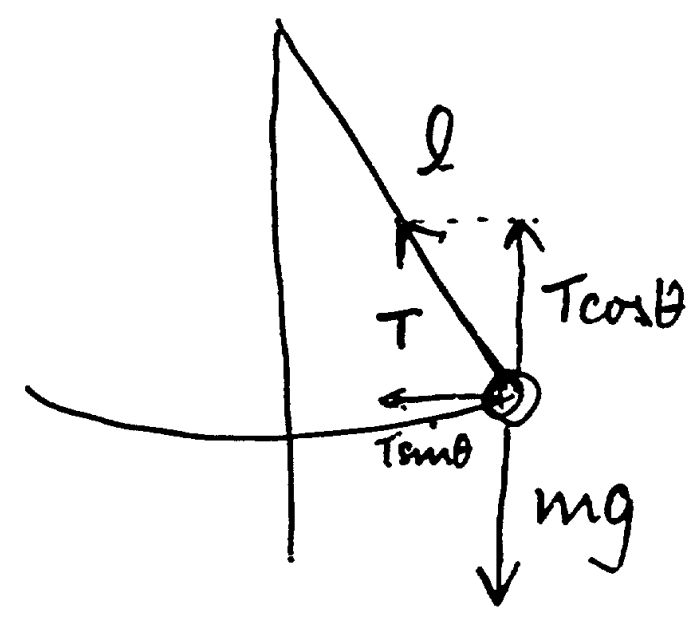

$$
\begin{aligned}
& m \ddot{x}=-T \sin \theta \quad \therefore \quad \therefore T=\frac{m g}{\cos \theta} \\
& m g=T \cos \theta \quad \therefore \quad
\end{aligned}
$$

Subs in (1)

$$
m \ddot{x}=-m g \tan \theta
$$

if $\theta \ll \pi / 2, \quad x=l \sin \theta \approx l \theta, \ddot{x}=l \ddot{\theta}$

$$
\begin{aligned}
& m \ddot{x}=-m g \theta \\
& \text { whl } \ddot{\theta}=-m g \theta . \\
& \ddot{\theta}+\left(\frac{g}{e}\right) \theta=0
\end{aligned}
$$

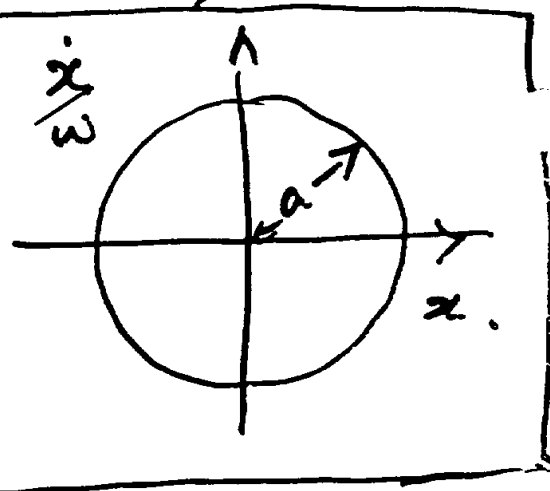

or $\ddot{x}+\left(\frac{p}{\rho}\right) \bar{x}=0$

$$
x=a \cos \left(\omega \frac{1}{2}+\lambda\right) \quad \text { whene } \omega=\sqrt{\frac{g}{2}} \text {. }
$$

check.

$$
\begin{aligned}
\dot{x} & =-a \omega \sin (\omega t+\lambda) \quad \frac{\dot{x}}{\omega}=-a \sin (\omega t+ \\
\ddot{x} & =-a \omega^{2} \cos (\omega t+\lambda) \\
& =-\omega^{2} \theta
\end{aligned}
$$

$+8$

2.1 
$\operatorname{soln} 2$

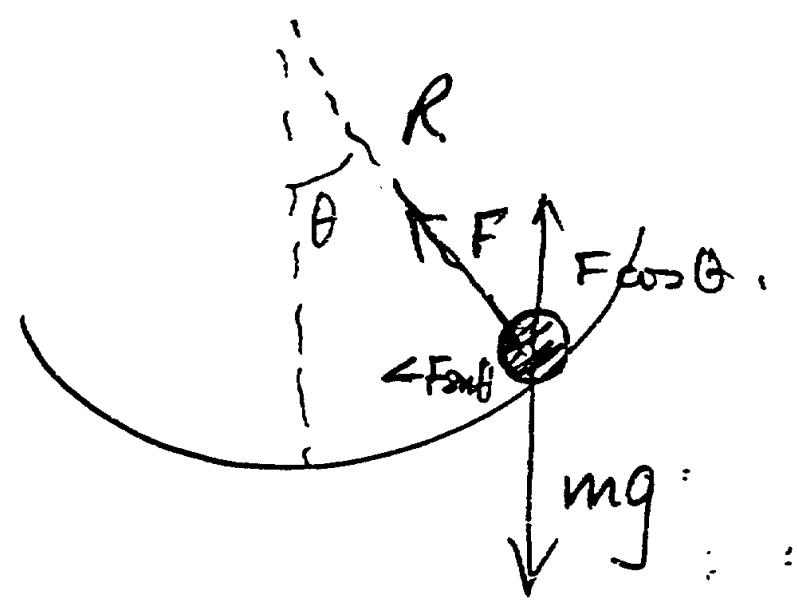

As before $m \ddot{x}=-F \sin \theta$

$$
\begin{aligned}
& m g=F \cos \theta \\
& \ddot{\theta}+\left(\frac{g}{R}\right) \theta=0 \\
& x \approx R \theta . \\
& \ddot{x}+\left(\frac{g}{R}\right) x=0 \\
& x=a \cos (\omega t+\lambda)
\end{aligned}
$$

but $s=$ distance along gutter $=$ it

$$
\begin{aligned}
& \frac{d^{*} x}{d t}=\frac{d x}{d s} \cdot \frac{d s}{d t}=v \frac{d x}{d s}, \frac{d^{2} x}{d t^{2}}=v^{2} \frac{d^{2}}{d s} \\
& \frac{d^{2} x}{d t}=\frac{d d^{2} x}{d t}(s) \\
& x^{\prime \prime}=\frac{d^{2} x}{d^{2}}=-\left(\frac{g}{d^{2} \cdot 2}\right) x
\end{aligned}
$$




$$
\begin{aligned}
& \text { if } \quad x=a \cos \left(2 \pi \frac{x}{\lambda}+\mu\right) \\
& x^{\prime}=-a \cdot \frac{2 \pi}{\lambda} \sin \left(\frac{2 \pi x}{\lambda}+\mu\right) \\
& x^{\prime \prime}=-a\left(\frac{2 \pi}{\lambda}\right)^{2}\left(\frac{2 \pi x}{\lambda}+\mu\right) \\
& =-\left(\frac{2 \pi}{\lambda}\right)^{2} x=-\left(\frac{g}{e v^{2}}\right) x
\end{aligned}
$$

$-$

$$
\begin{aligned}
& \frac{2 \pi}{\lambda}=\frac{1}{v} \sqrt{\frac{g}{R}} . \\
& \lambda=2 \pi v \sqrt{\frac{R}{g}}
\end{aligned}
$$

$$
\text { wane number }=\frac{l}{\lambda}=\frac{1}{2 \pi} \cdot \frac{l}{\left(v^{2} R / g\right)^{1 / 2}}
$$

Analogy for synchrotron.

$$
Q=\frac{1}{2 \pi} \int \frac{d s}{\beta}
$$

$$
\rho^{1 / 2} \equiv \frac{\lambda}{2 \pi} \text { locally. }
$$

80 
Sol.' 3

a)

$$
\begin{aligned}
& x=a \cos \omega t \\
& \dot{x}=\frac{d x}{d t}=-a \omega \sin \omega t
\end{aligned}
$$

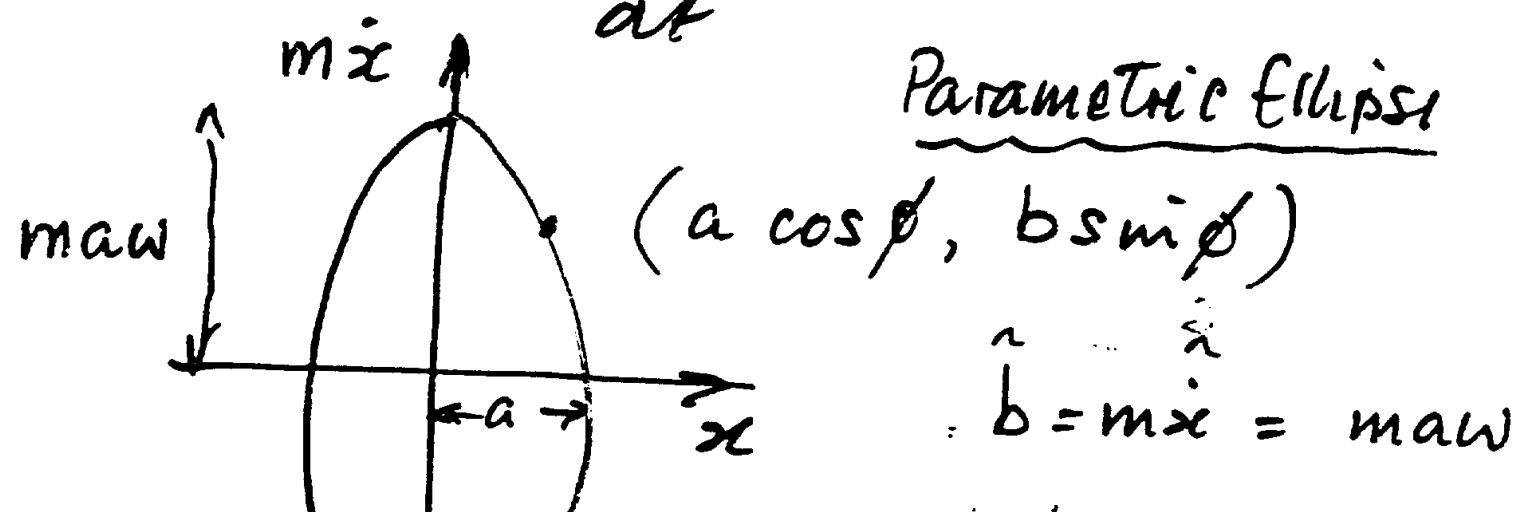

$\operatorname{Soln} 4$$$
x=a \cos 2 \pi\left(\frac{x}{\lambda}\right)
$$$$
\lambda=2 \pi v \sqrt{\frac{R}{g}} \text {. }
$$$$
\therefore x=a \cos \left(\frac{1}{v} \sqrt{\frac{g}{R}} \cdot x\right)
$$$$
x^{\prime}=-\frac{1}{V} \sqrt{\frac{g}{R}} \sin (\quad)=b \sin (1
$$

Cinglatul.

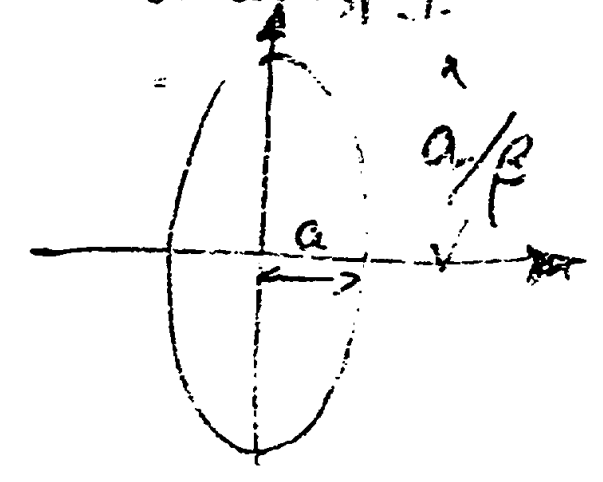

Qt.2.24 
Sthe

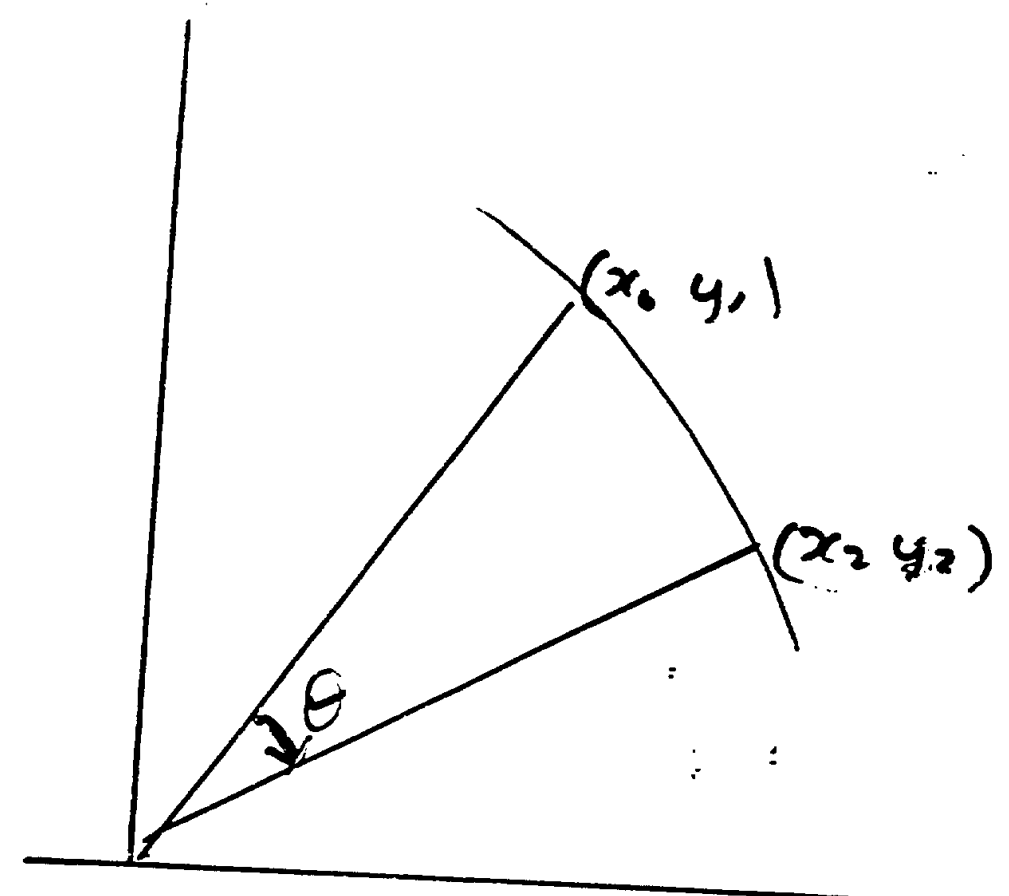

Same as retating coordunates - 6

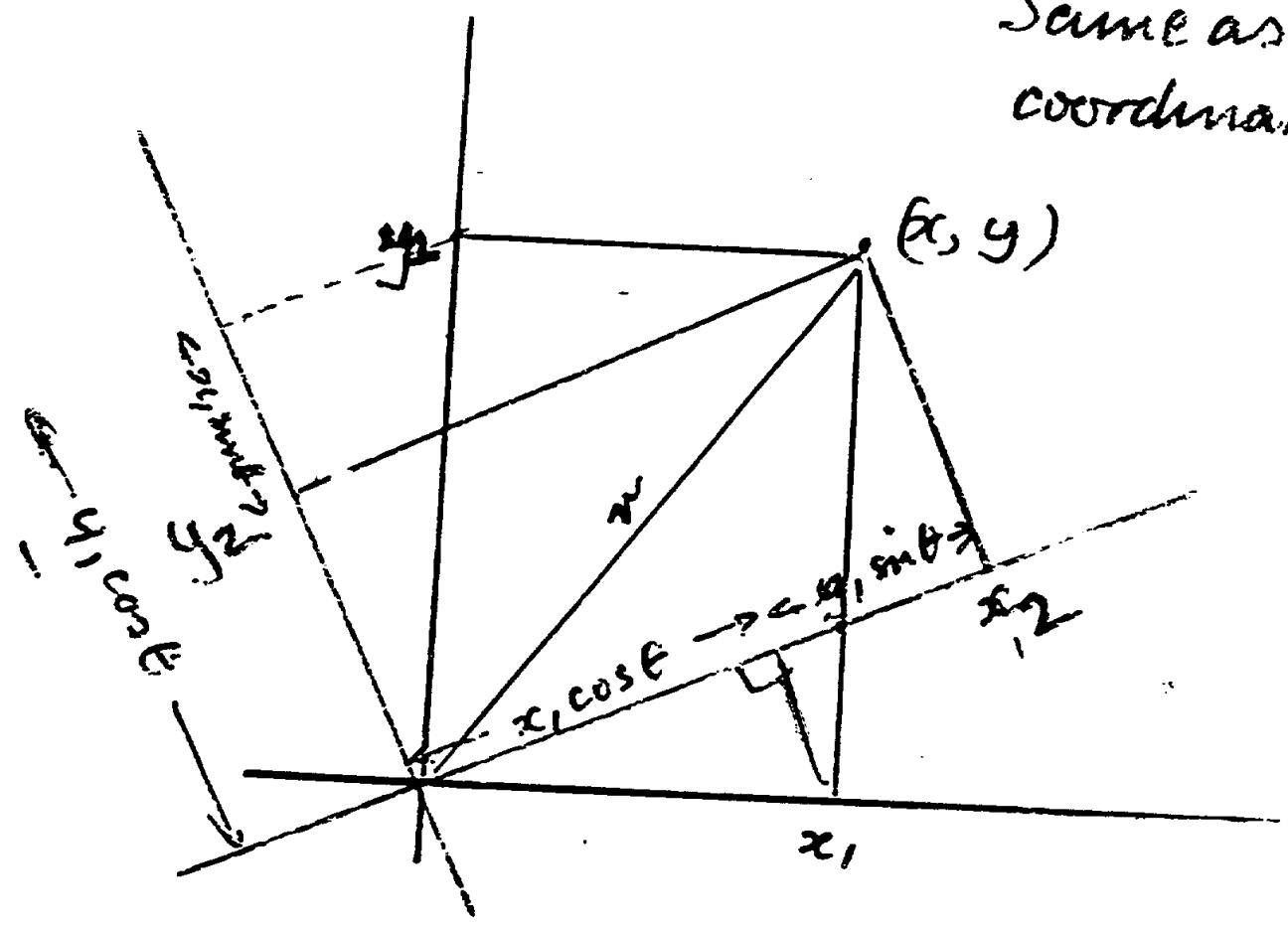

a)

$$
\begin{aligned}
& x_{2}=\cos \theta x_{1}+\sin y_{1} \\
& y_{2}=-\sin x_{1}+\cos y_{1}
\end{aligned}
$$

c)

$$
\left(\begin{array}{l}
x_{2} \\
y_{2}
\end{array}\right)=\left(\begin{array}{ll}
\operatorname{ent} & \sin \\
-\tan t & c_{1}=6
\end{array}\right)\left(\begin{array}{l}
x_{1} \\
y_{1}
\end{array}\right)
$$

2.25 
Sol". 5

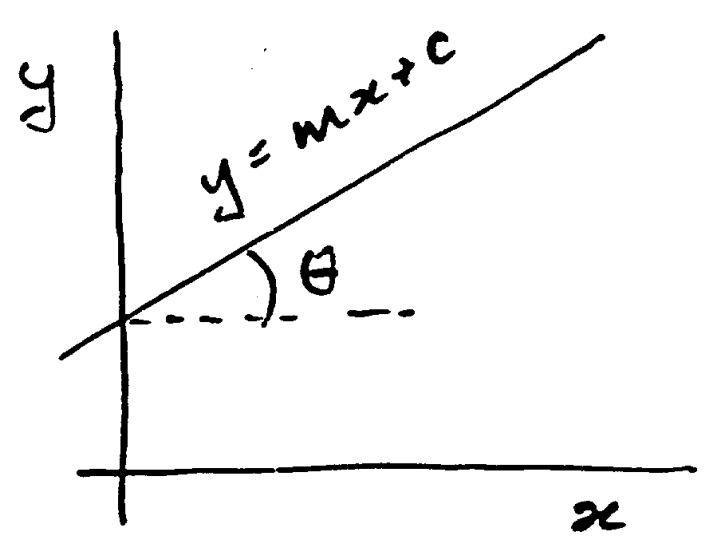

Maths Books

$$
\begin{aligned}
& y=m x+c \\
& \theta=\tan ^{-1} m \\
& \left.m=\frac{d y}{d x} ;=x^{\prime}\right)
\end{aligned}
$$

Accelerator Books

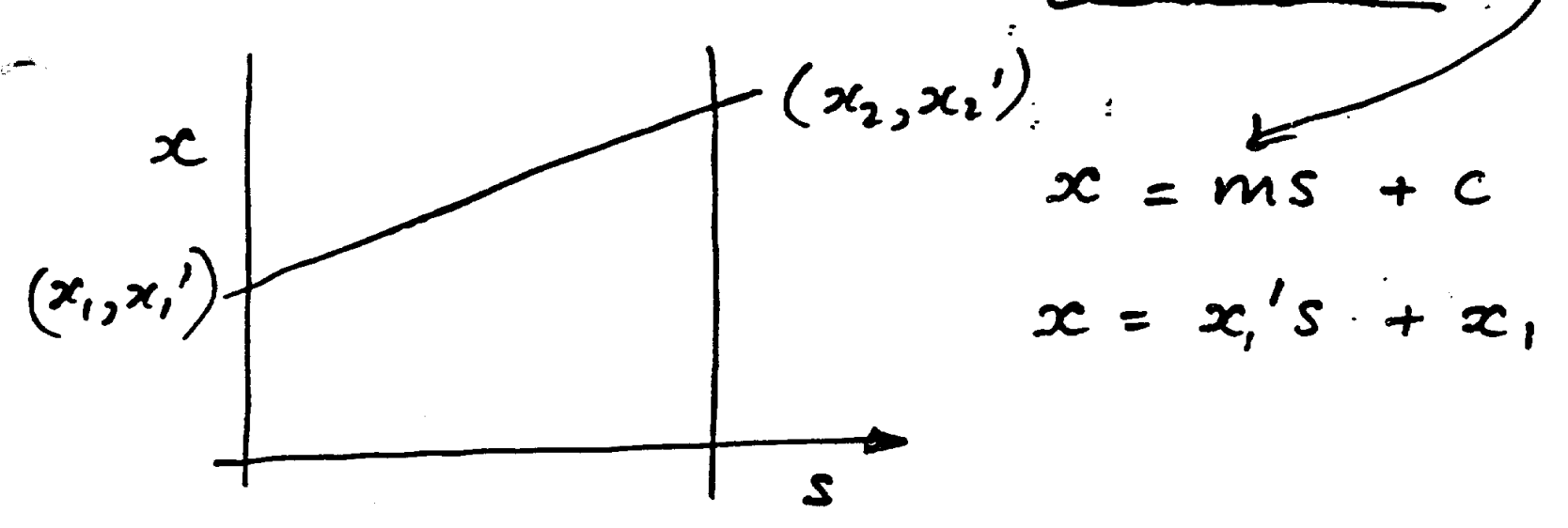

a) $\theta=\tan ^{-1}\left(x_{1}^{\prime}\right)$

b)

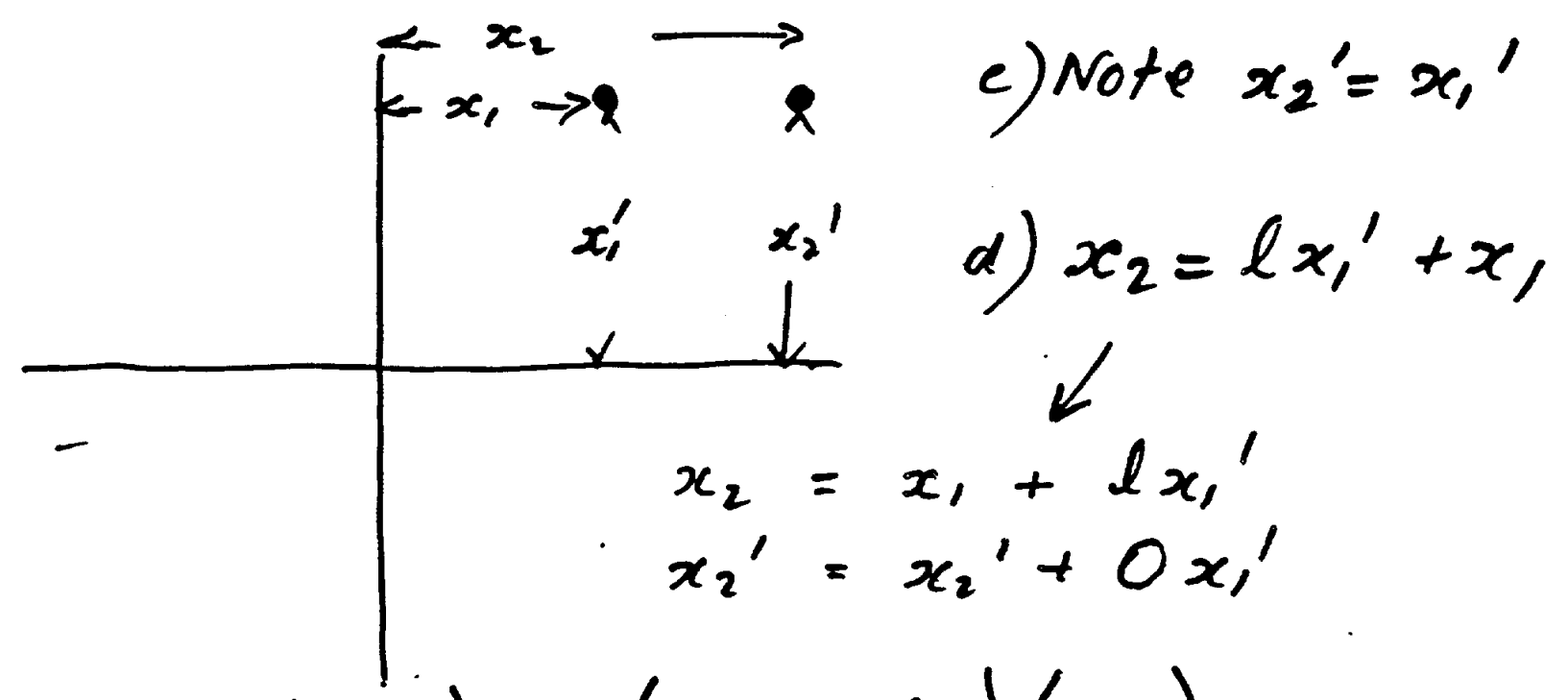

e)

$$
\left(\begin{array}{l}
x_{2} \\
x_{2}^{\prime}
\end{array}\right)=\left(\begin{array}{ll}
1 & l \\
0 & 1
\end{array}\right)\left(\begin{array}{l}
x_{1} \\
x_{1}^{\prime}
\end{array}\right)
$$

qr

$2.2 k$ 
a)

$$
\begin{aligned}
\operatorname{det} M & =m_{11} m_{12}-m_{2,1} m_{12} \\
& =\cos ^{2} \theta-\left(-\sin ^{2} \theta\right) \\
& =\cos ^{2} \theta+\sin ^{2} \theta=1
\end{aligned}
$$

e)

$$
\begin{aligned}
& \theta \rightarrow-\theta \cdot \\
& \left(M^{-\theta}\right)=\left(\begin{array}{cc}
\cos \theta & -\sin \theta \\
\sin \theta & \cos \theta
\end{array}\right)
\end{aligned}
$$

Formally

$$
\left(M^{-1}\right)=\frac{1}{|\operatorname{det} M|}\left(\begin{array}{c}
a_{i j}-. . \\
\vdots
\end{array}\right)
$$

ain is thu submatrix (or its determinant? if you cross out the $i$ th row and i th colum,

-

24

2.2 
Solution 7

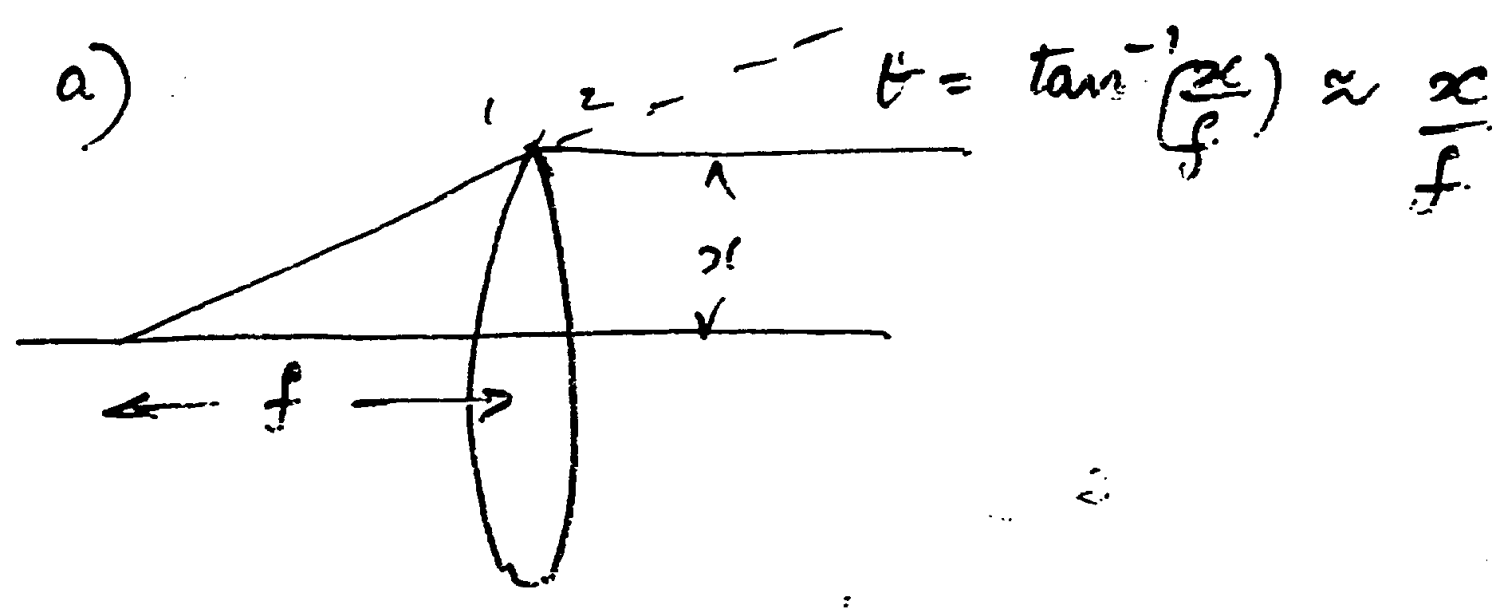

b) $y+5$

c)

$$
\begin{aligned}
& x_{2}=x_{1}+0 x_{1}^{\prime} \\
& x_{2}^{\prime}=-\frac{x_{1}}{f}+x_{1}^{\prime} \\
&\left(\begin{array}{l}
x_{2} \\
x_{2}^{\prime}
\end{array}\right)=\left(\begin{array}{cc}
1 & 0 \\
-\frac{1}{f} & 1
\end{array}\right)\left(\begin{array}{l}
x_{1} \\
x_{1}^{\prime}
\end{array}\right)
\end{aligned}
$$

d) yer.

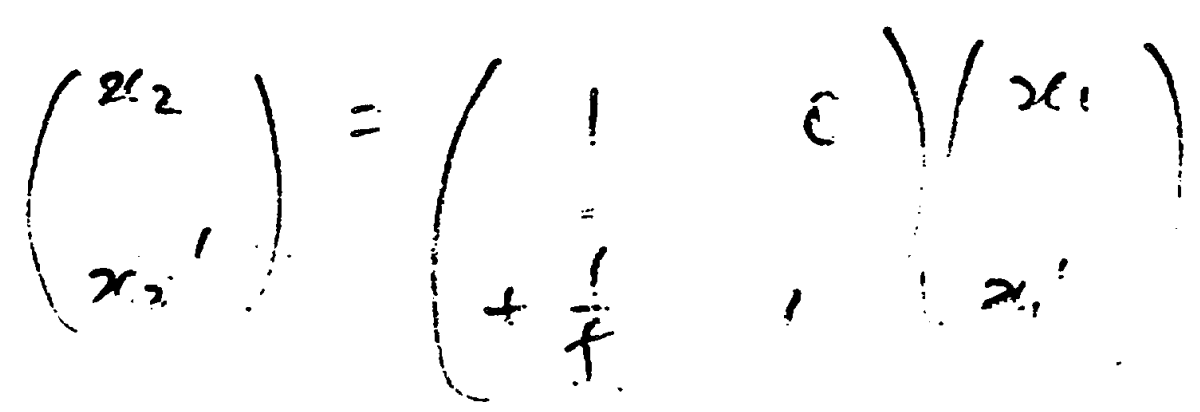

25 
Jol! 8

(8)

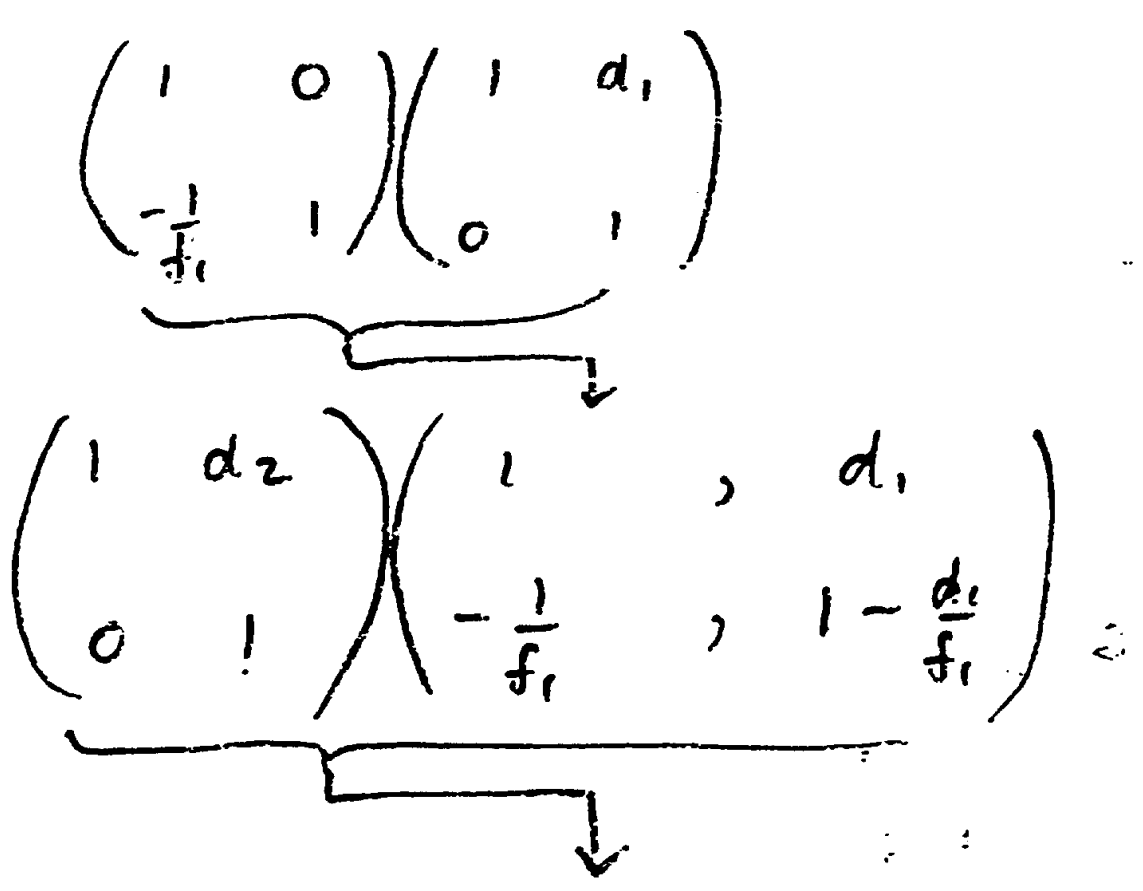

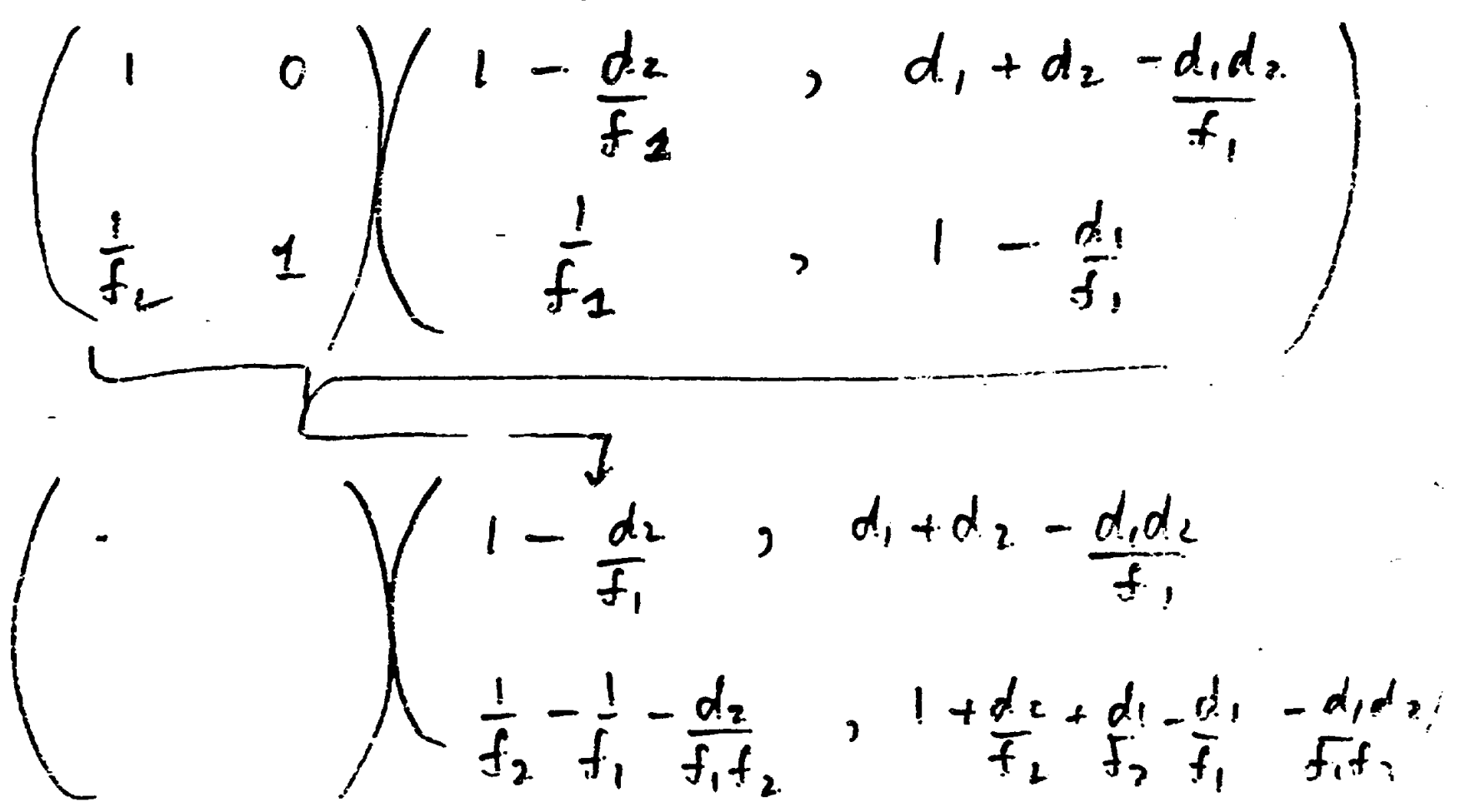

$t_{2.29}$ 

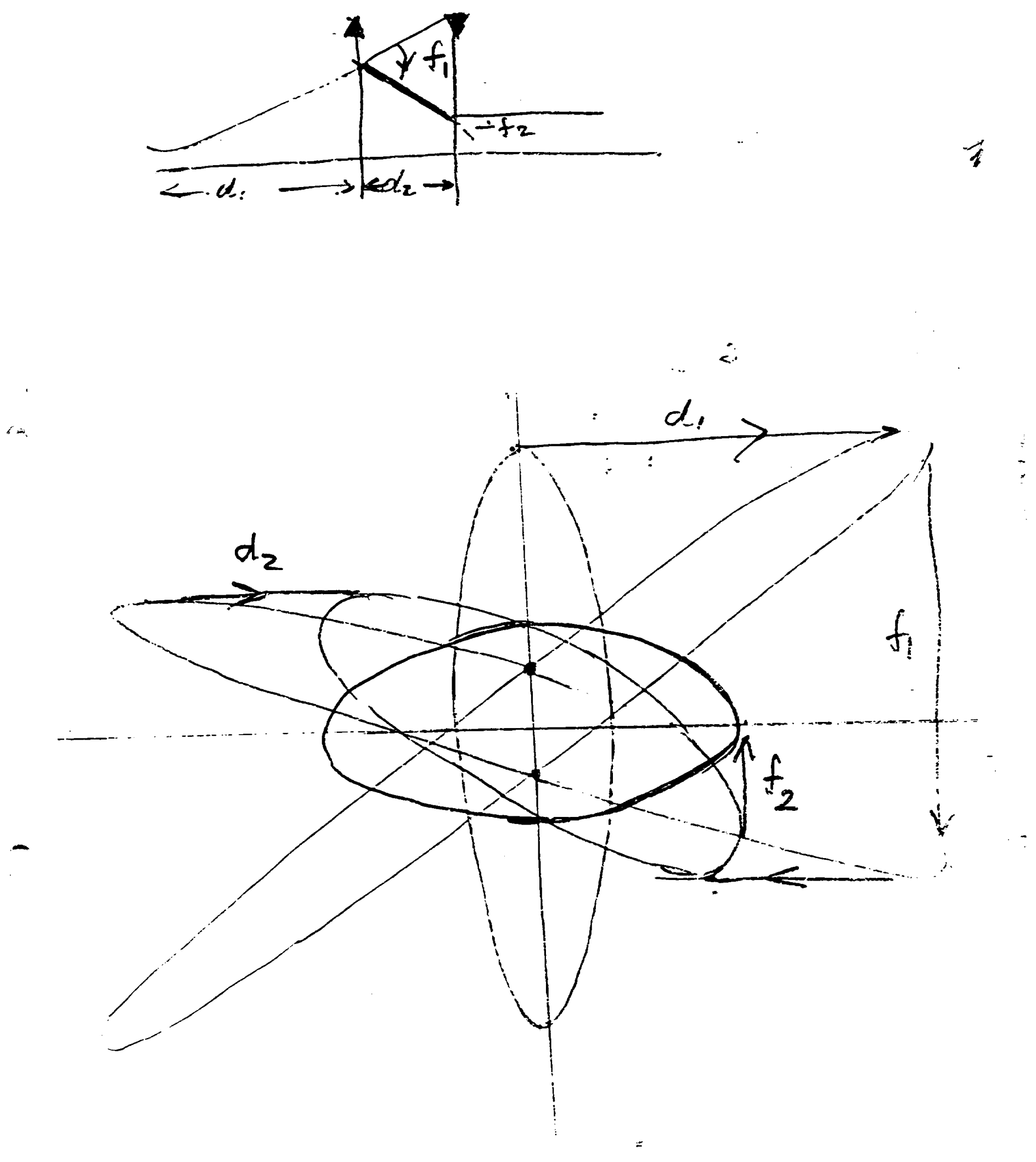
Solution 9

a)

$$
\text { a) } \begin{aligned}
\dot{x} & = \pm j \omega a e^{ \pm j \omega t} \\
\ddot{x} & =( \pm j \omega)^{2} a e^{ \pm j \omega t} \\
& =-\omega^{2} a e^{ \pm j \omega t} \\
& =-\omega^{2} x \\
\therefore \quad \omega & =\sqrt{k} .
\end{aligned}
$$

b) $x=a e^{ \pm j w s}$

snick the solution is periodic every time $s$ increases by $\frac{2 \pi}{\omega}$ the wavelengfin is $\frac{2 \pi}{\omega}$.

28 2.3 


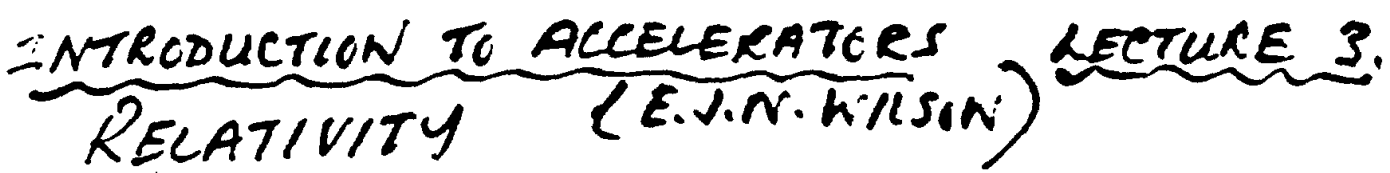

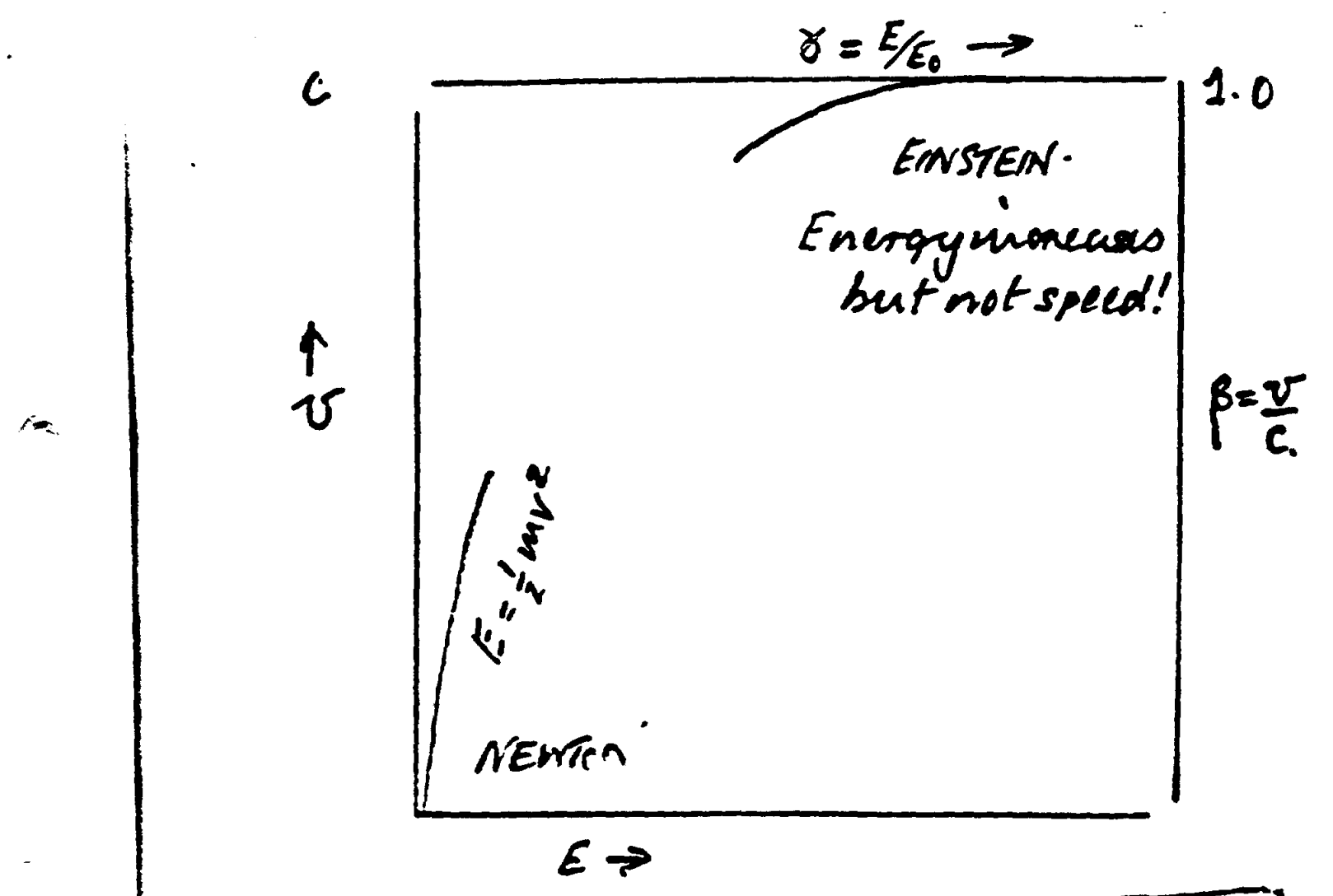

Energy of a particle at rest $=E_{0}=m_{0} c^{2}$

Total Guergy of a Moving Particle

$$
\text { (defuiction of } \gamma \text { ) } E=\gamma E_{0}=m, e^{2} \gamma
$$

Another ulatursitic varable is defuid:

$$
\beta=\frac{\text { moneentum }}{\text { snergy }}=\frac{p e}{E}=\frac{v}{c} \text {. }
$$

Solve thesy equations to get

$$
P=\frac{E \beta}{c}=m_{0} c(\beta \gamma)
$$

$\zeta$ ised in $r$ plose plits. 


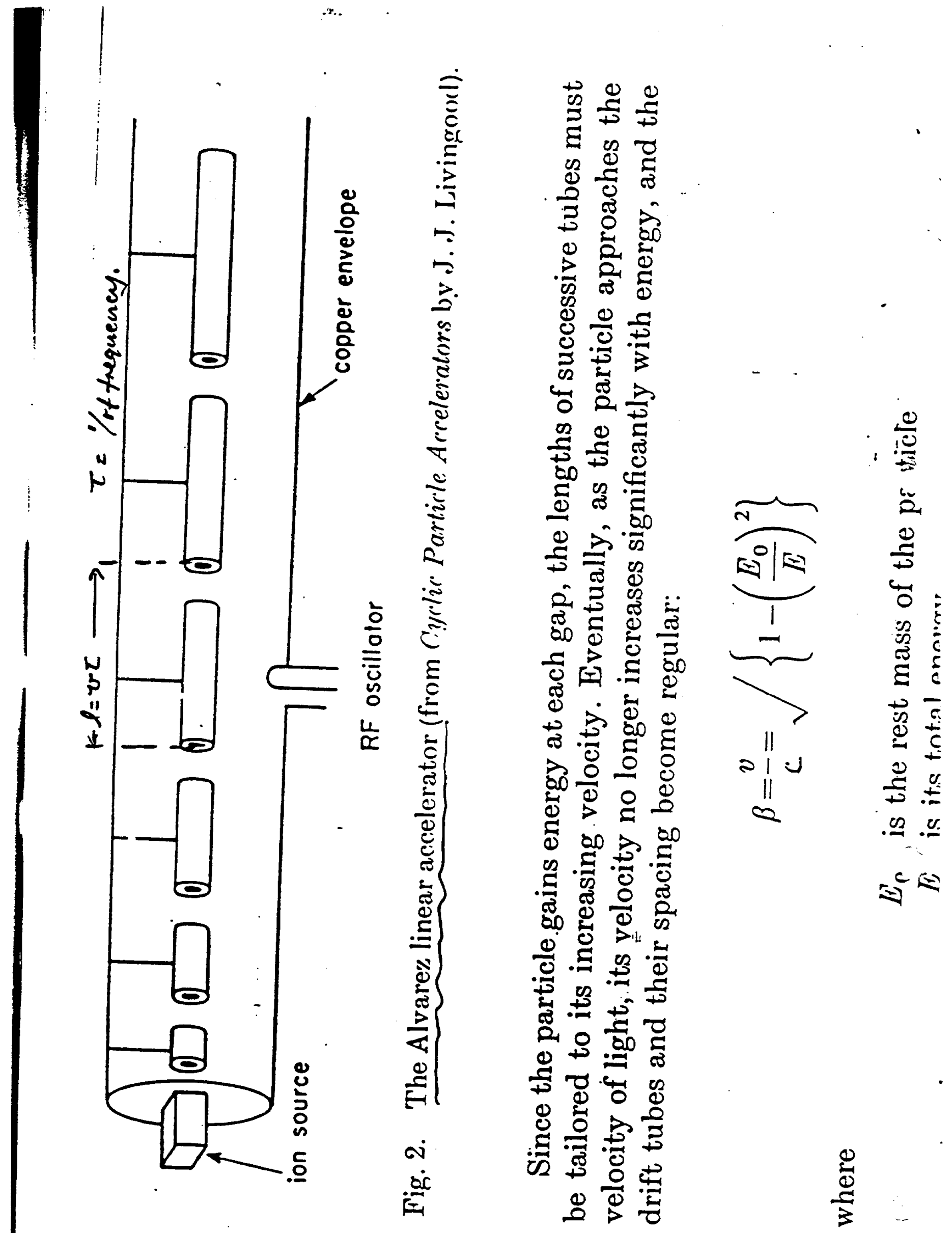


PHASE STABILITY

(A) is the synchronous particle - gets the right amount of energy:

$$
V=V_{0} \sin \phi_{s}=\frac{\text { energy }}{\text { turn }}
$$

to follow the rising $S$.

(3) is late - in meantime rif wave has grown so it gets more volts than $A$ - speeds up - and catches it

Fig. 22 Cylindrical coordinate system rotates with beam, demonstrates meaning of RF phase angle in longitudinal phase space

$$
N B f=\frac{\beta C}{2 \pi R} .
$$

In fact $B$, overtakes $A$, overshoots and -oscillates about $A$.

In phase space $(\Delta \beta \gamma, \phi)$

it follows an ellipse and makes phase.

oscillations with frequency:

F. If which defines a number of oscillations / machine turn:

$$
\nu_{s}\left[\text { or } Q_{s}\right]=f_{s} / f_{0} \text {. }
$$

Usually $\ll, \approx 0.05$

3.3 


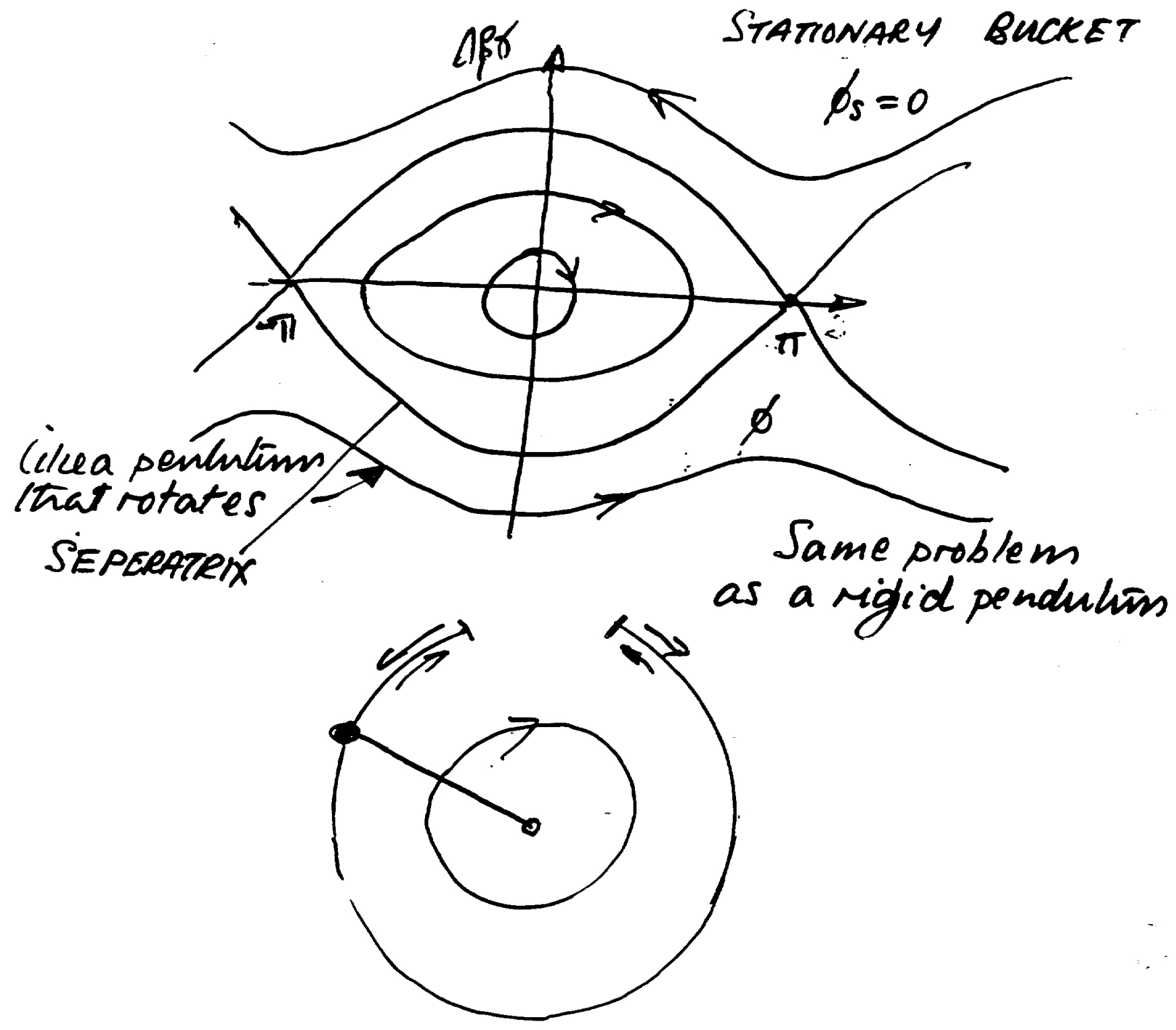

BUCKET AREA AND PENDULUM ANALOGY.

3 
MAGNETIC RIGIDITY

Force $=$ (centripetal ) acceleration

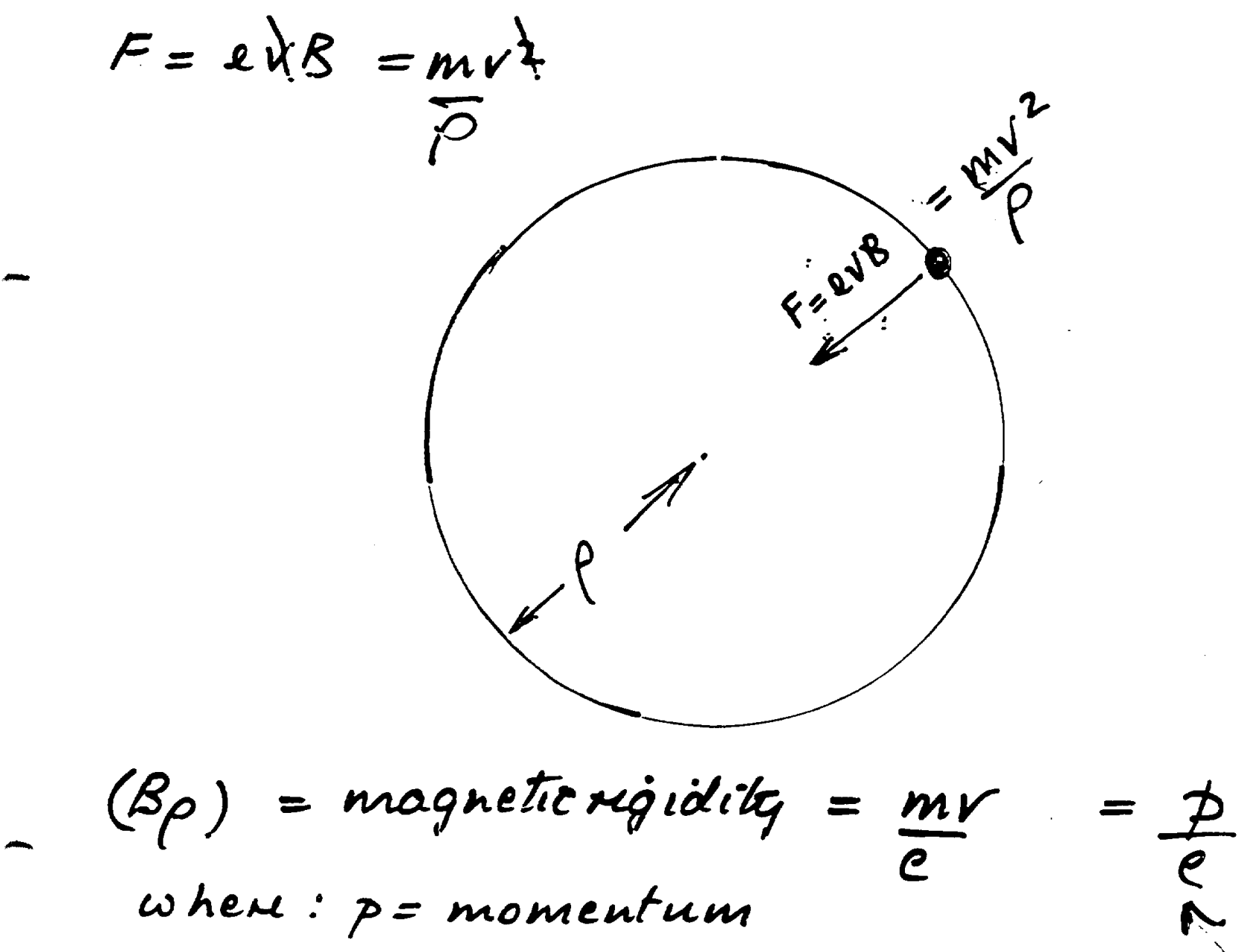

Fortunately as $r \rightarrow c$ this is also true !

With all the conversion factory

$(B \rho)=3.3356 \times \not \supset$

[Tesla.m] [GeV/c]

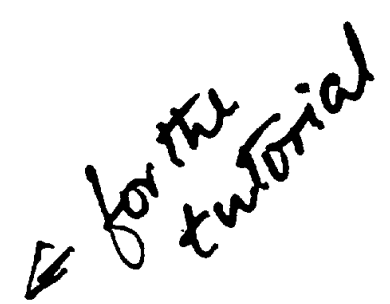

Add us all the bending magnets in the ring $B \times$ Number $\times$ length $=2 \pi \rho B=2 \pi \times 3.3556 \times p$ 


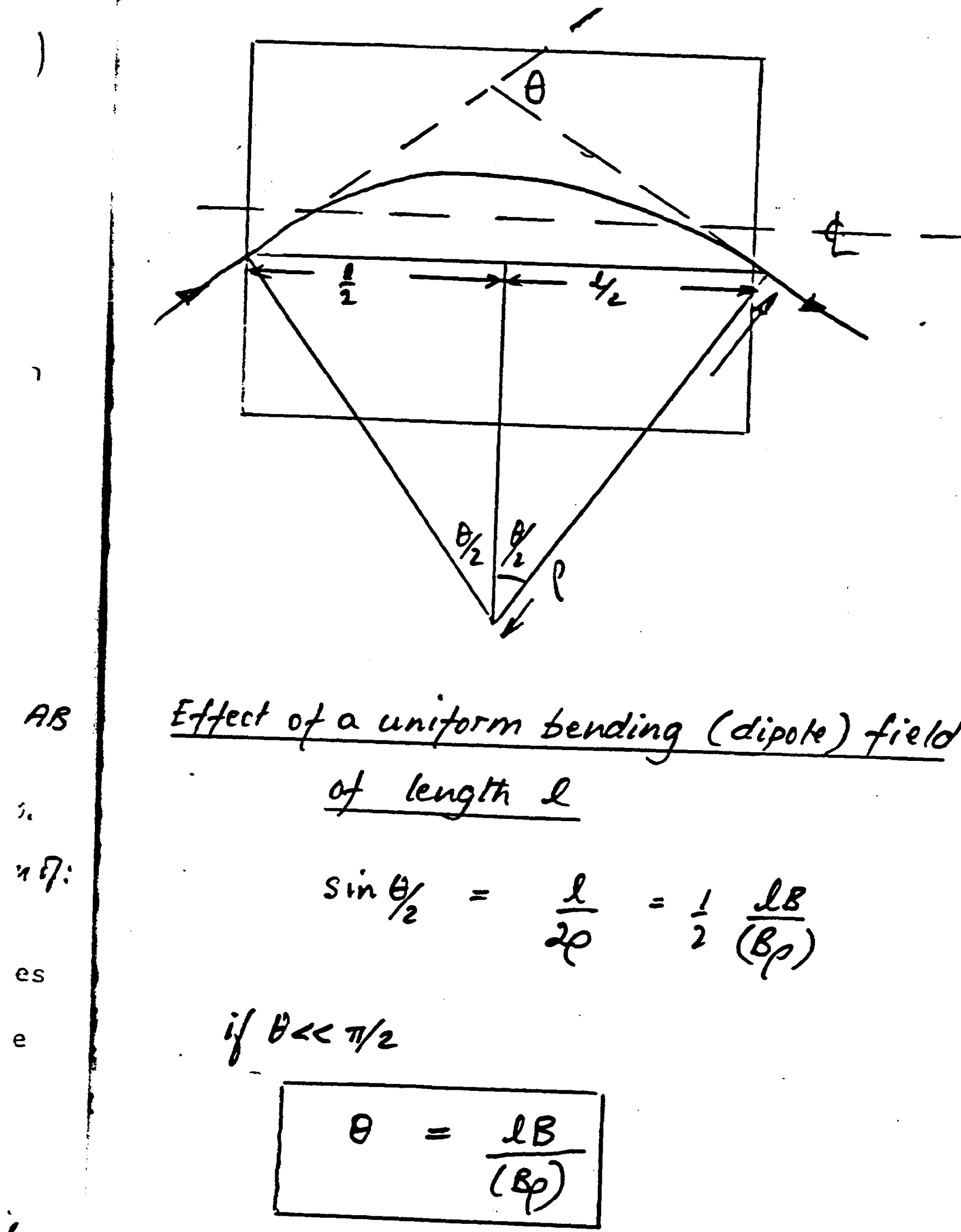

in,

18 


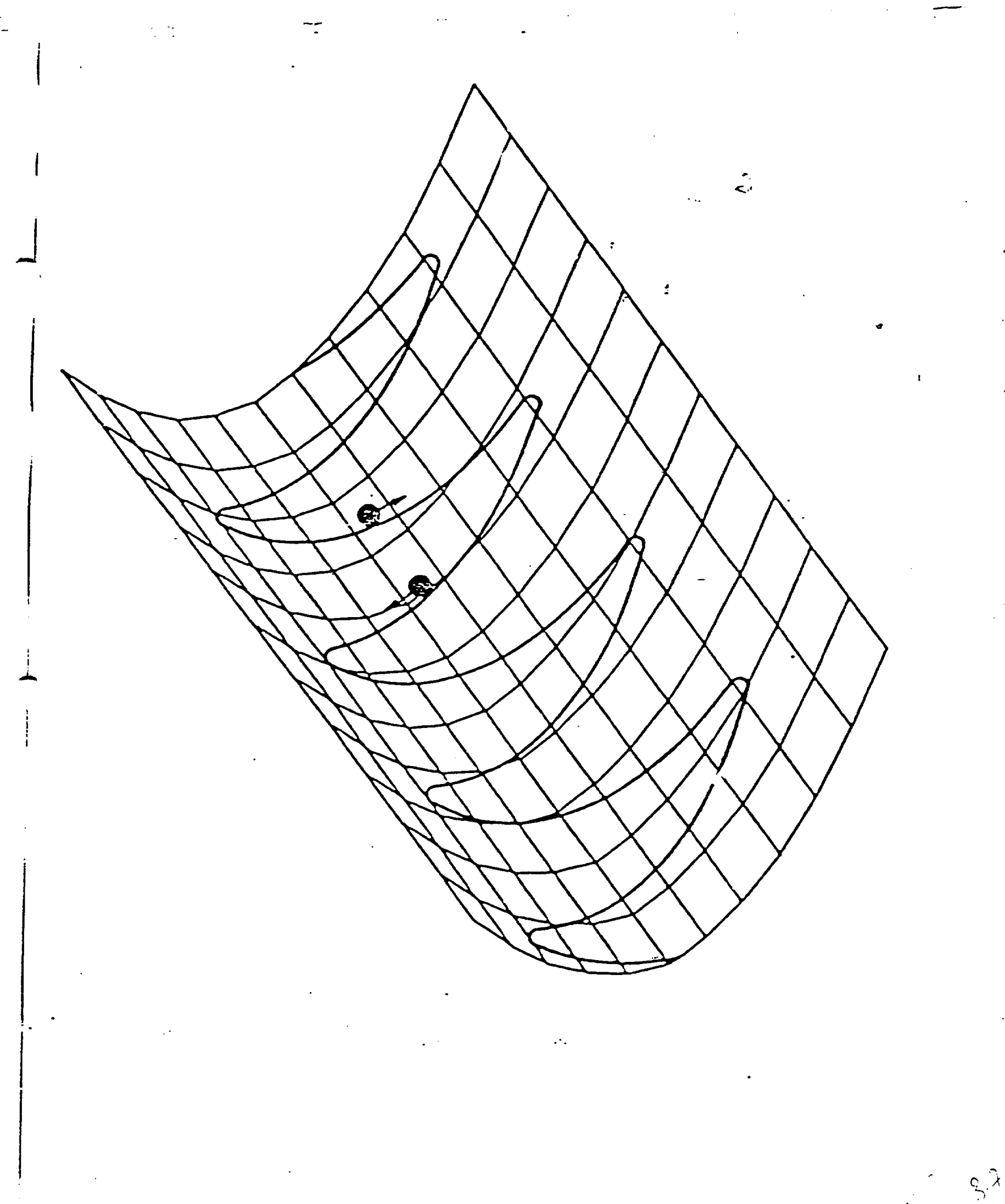


TranSVERSE MCTICN IN PHASE SPACE $\left(x_{2}\right.$ :

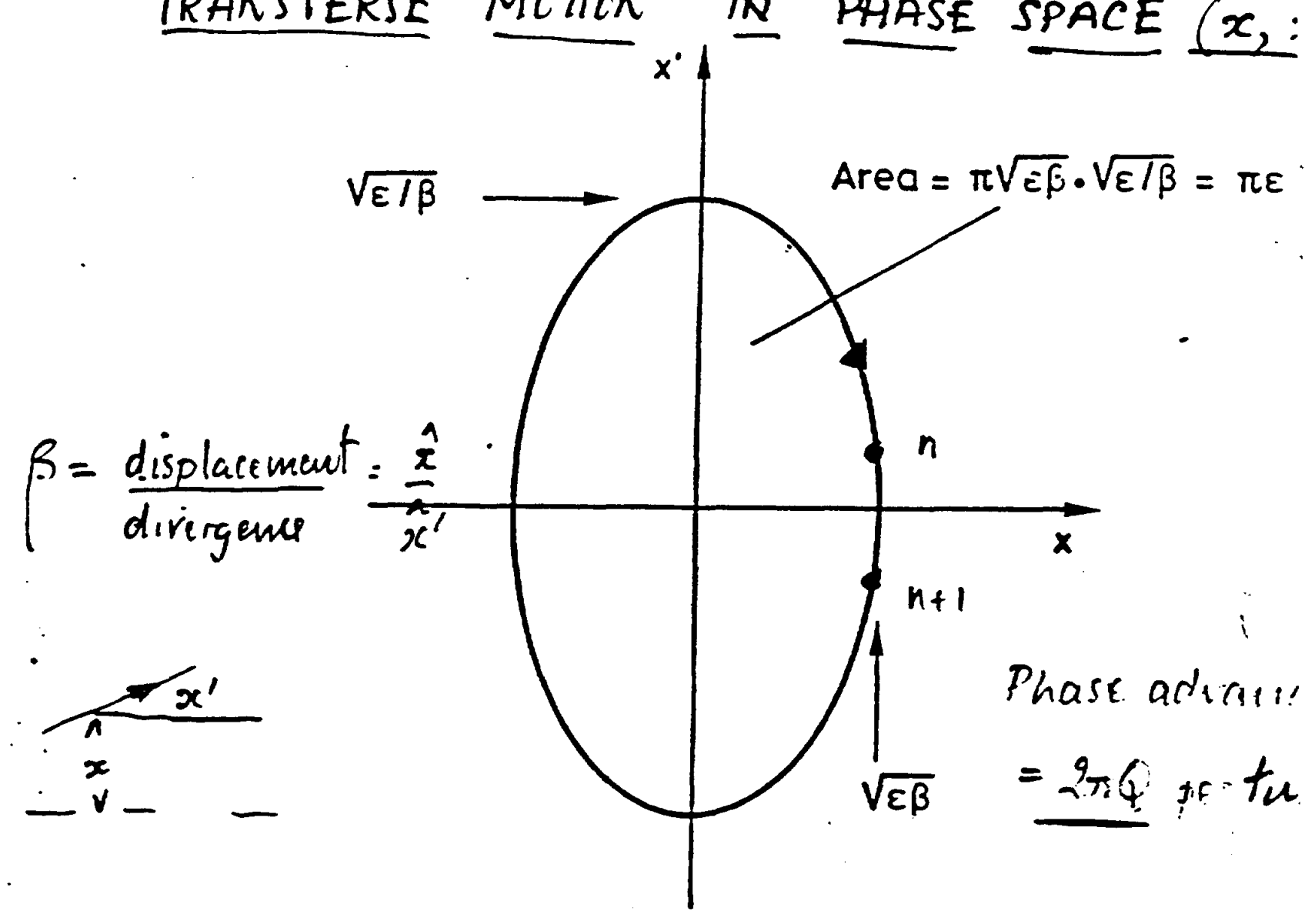

Imagine a circular. gutter around which the marble wobbles. Measure and plot the clesplacement $x$ and dwergence angle $x$ ' ( $\alpha$ velvety in $x$ direction). If phase is different on each turn measurements coll die on an ellipse. (Just a description of SAM)

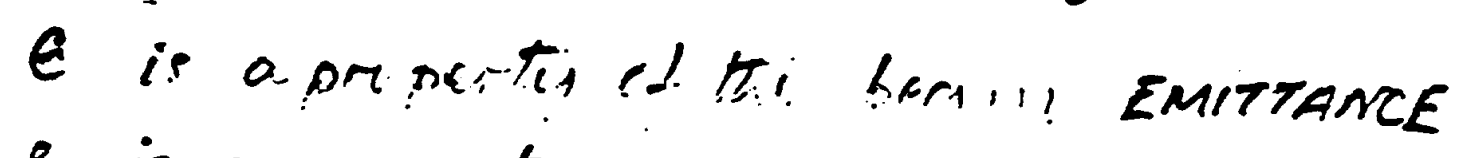
$\beta$ is a property of the focusing system and will vary around the ring for $A G$ focusing, changing aspect ratio of ellipse and 7 tilting it depending on where you sit. 7 
EFFECT OF A QUADRUPLE FOCUSING MARNE?

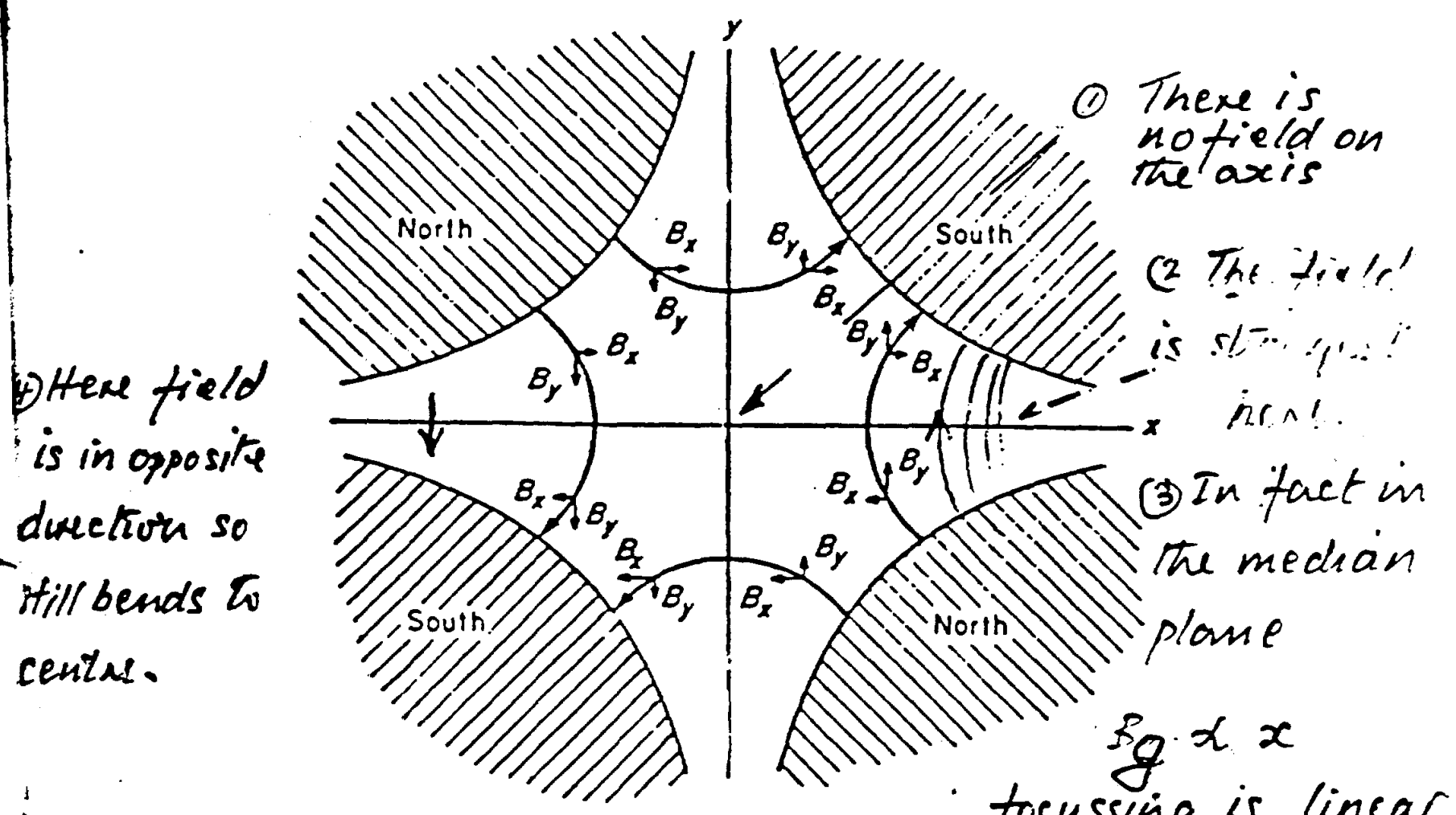

$\therefore F_{x}$ is towards $x=0$

$\therefore$ focussing is linear ind focussing it fy is away in axis Fin Hplane Din replace.

i RLTERNATINC GRADIENT FoCUSSING is USED

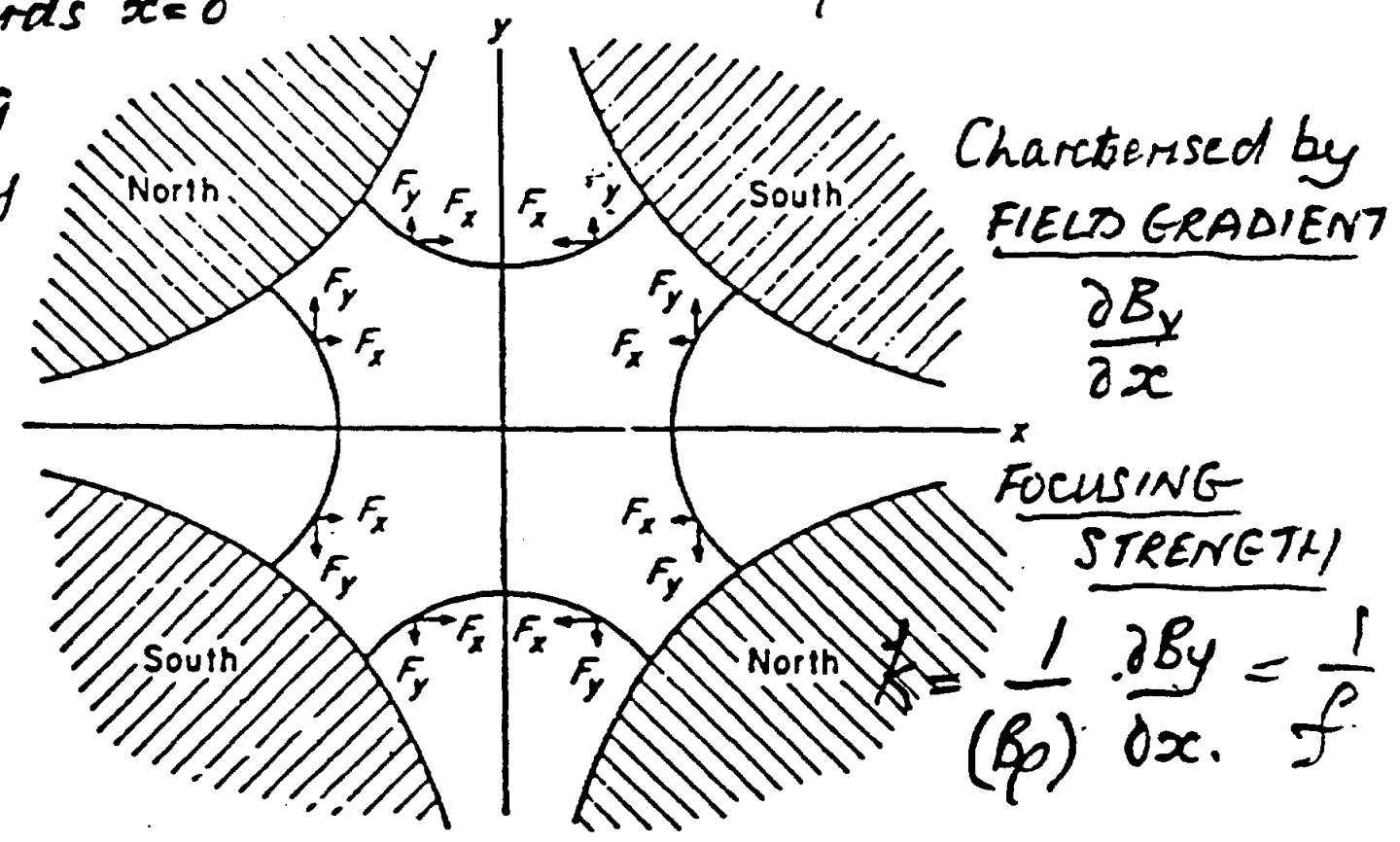

Components of field and force in a magnetic quadrupole. Positive ions approach lite reader on paths parallel to the $\boldsymbol{s}$ axis (from ('yrlir Particle Arrelernlor: by .I. . ? livingoul). (This is such a nice drawing $I$ did net want to change all the y's to z)

z

5

$\psi$

9.9 
AG. Focussing
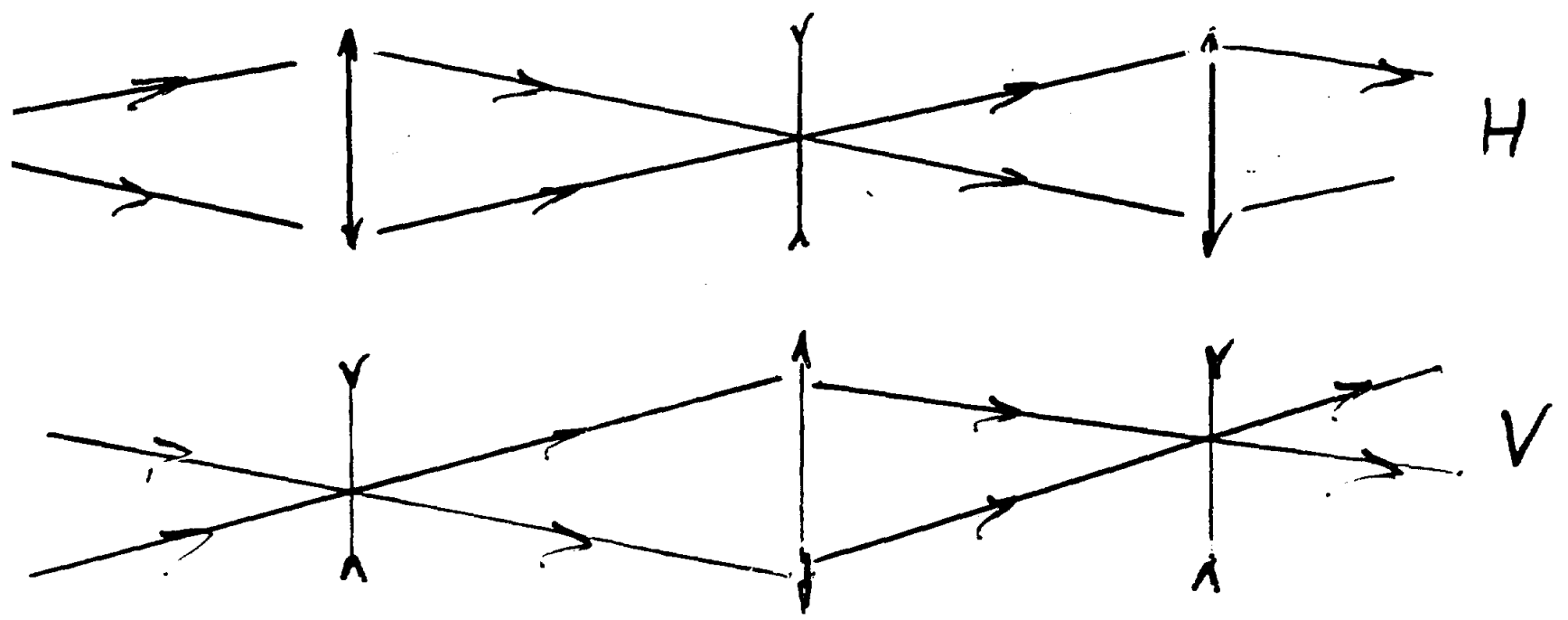

(a) Optical analogy - lenses which are concave in one plane and convex in the other. - the defocusing lenses have zero effect since rays pass down their centre.

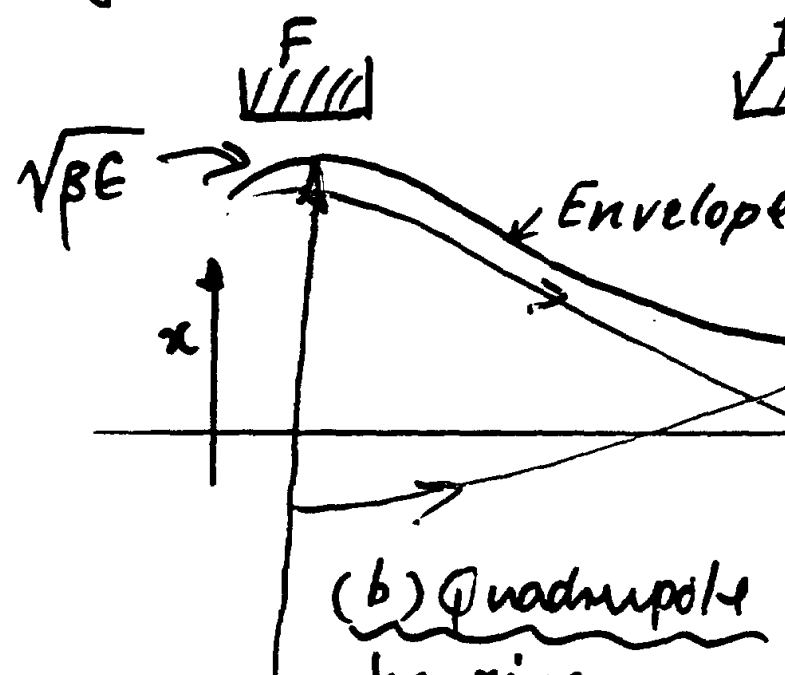

focusing

Here beam is weds and parallel (smal ldivergence)

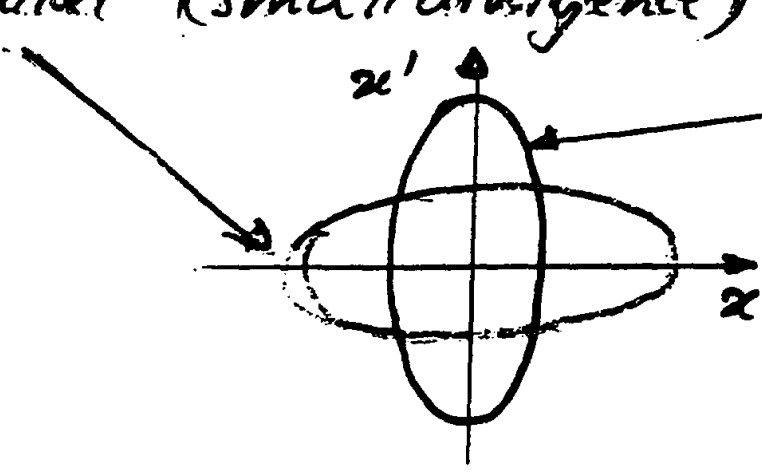

E II

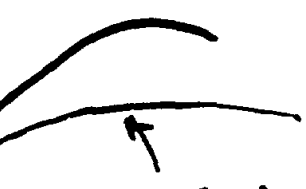

Trajectories

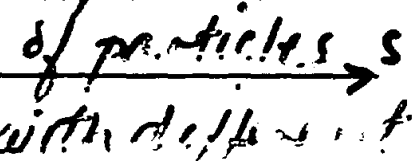

phases

At this focus' or waist the beams has a large diverging but is narrows.

( 
EQUATION OF MOTION OF PARTICLES IN TRANSVERSE FOCUSING

(2.5) HILLSS EQUATION SYSTEM

Quadrupole strength

$$
\frac{d^{2} y}{d s^{2}}+k(s) y=0
$$

- This is a linear equation with periodic coffecient:

Ks) which is the focussing strength distributed around the ring (This is like the restoring constant in simple harmonic motion)

- The solution is not unlike SHM but the amplitude, which we call $\sqrt{k-\beta}$ and the phase $\phi$ are functions of $s$. I hus:

$$
y=\sqrt{\epsilon} \cdot \sqrt{\beta(s)} \cos [\phi(s)+\lambda]
$$

Look care fully -each term has a physical sristicance

$\sqrt{E}$ is this BEAN'S emittance

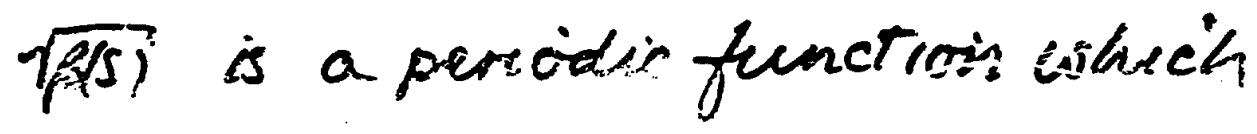
clescribe the machine LATTICE

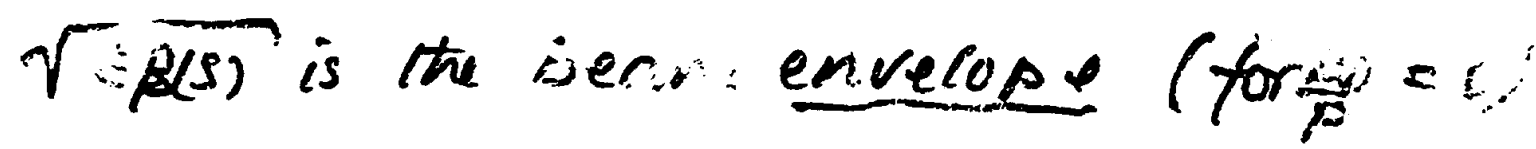

$\sigma[\phi(s)+\lambda]$ is line oscillation of the:

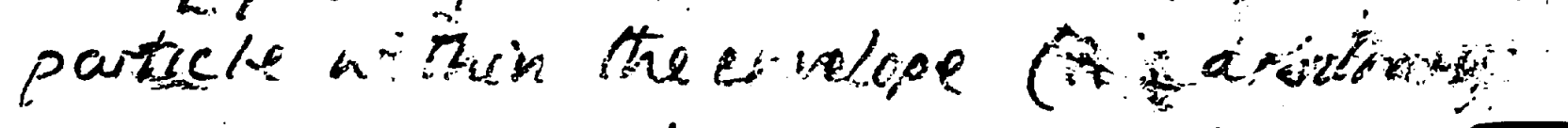

- Also

$$
\phi(s)=\int \frac{d s}{\beta(s)},
$$

$$
\begin{aligned}
& \hat{y}=\sqrt{\epsilon(s)}, \hat{y}^{\prime}=\sqrt{\epsilon /(s)} \\
& 2 \pi Q=6 \phi(s) d \varphi d t Q_{0} \phi_{0} ;
\end{aligned}
$$


$\rightarrow$ AARHUS 1989

(2.7) This is a $64 \mathrm{~m}$ long PERIOD of the CERN Super Proton Synchrotron (about $10 \%$ of the ring)

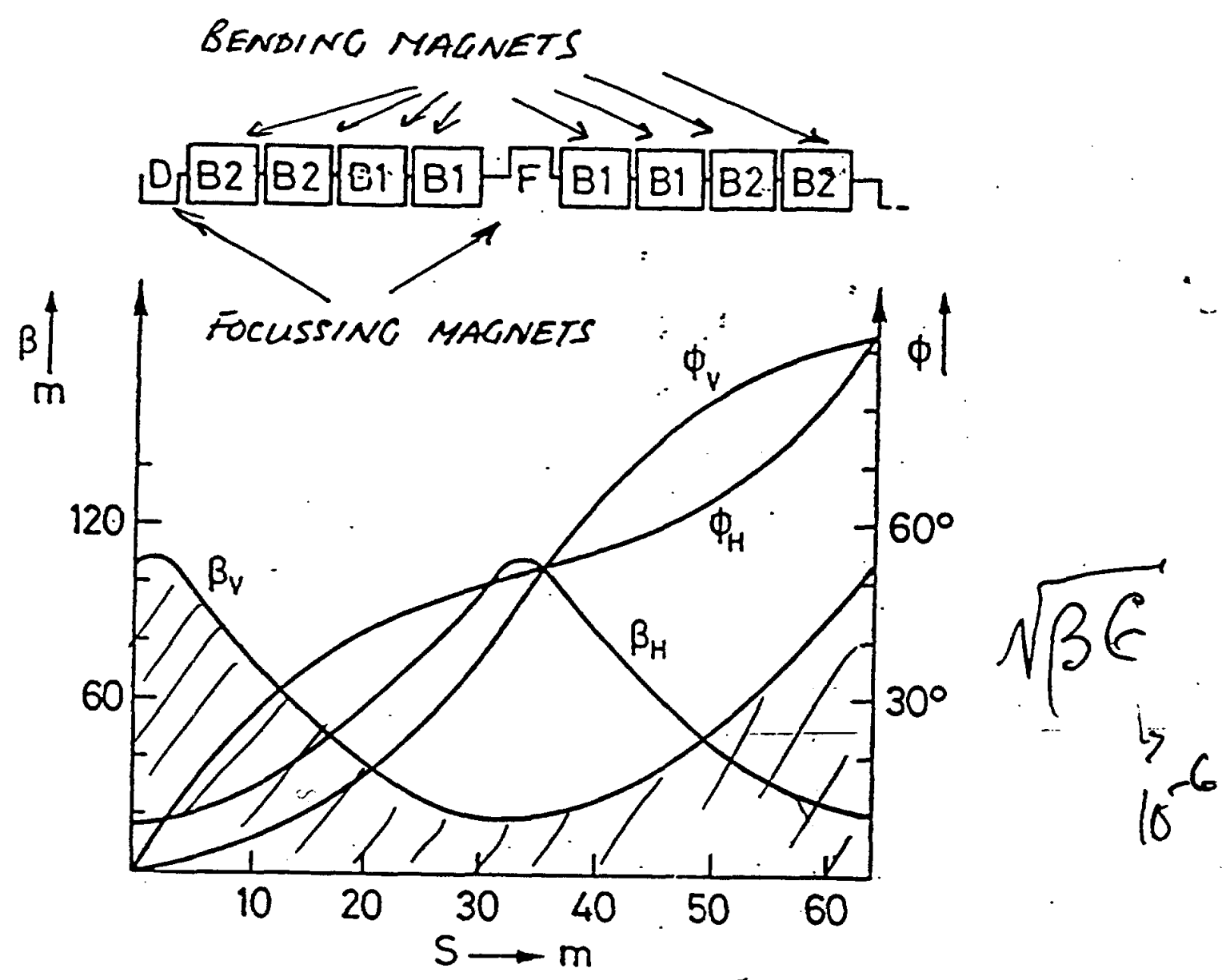

The functions $\beta$ (vertical) and $\beta$ (horizontal) describe the focussing properties of the machine. and define tie beam size.

They define the ENVELOPE of Trajectories which oscillate within them in a distorted form of simple harmonic motion which advances in phase as $\phi_{4}$ and $\phi_{v}$ 


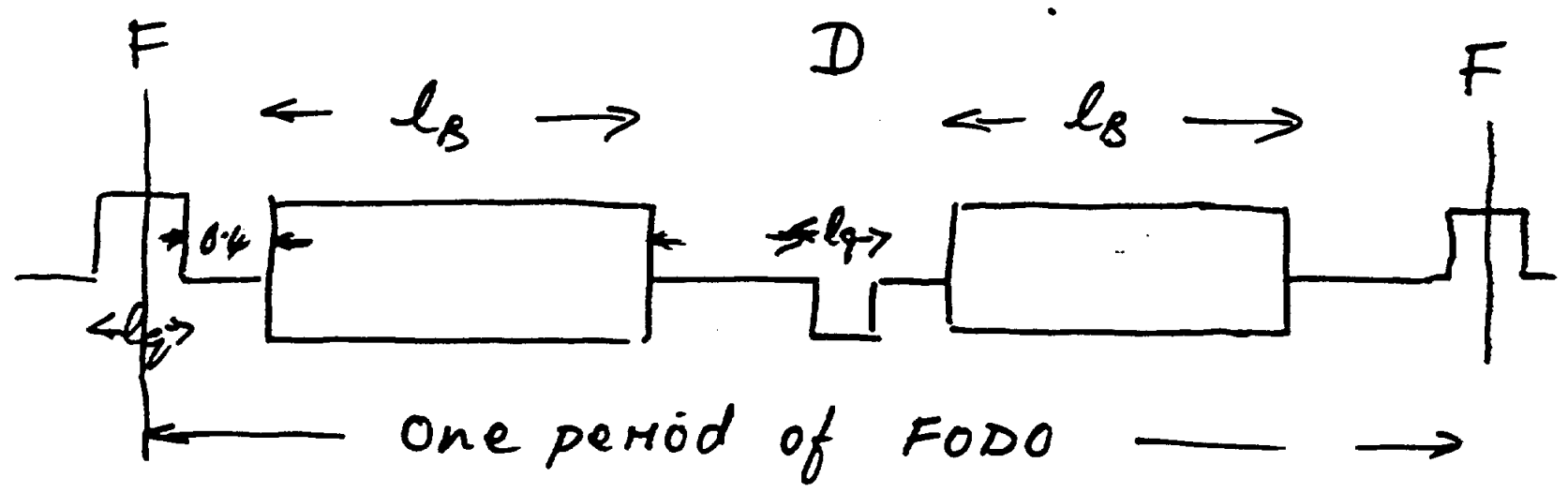

$$
B \times N_{B} \times l_{B}=2 \pi(\rho B)=2 \pi \times 3.3356 p[\mathrm{GNV} / \mathrm{C}]
$$

Frenoids $\times\left[\frac{l_{g}}{2}+0.4+l_{B}++l_{q}+0.4+l_{s}+\ldots l / 2\right]$ $=2 \pi R$.

Normaleells with bending maguets

Emptycells - Justonads. 


\section{TUTORIAL QUESTIONS FOR LECTURE \#3}

1. Find an expression for beta $(\mathrm{v} / \mathrm{c})$ as a function of Momentum (eV/c) and plot it for momenta in the range $1 \mathrm{GeV} / \mathrm{c}$ to $100 \mathrm{GeV} / \mathrm{c}$ for protons.( on log paper). What is the value for $8.0 \mathrm{Ge}$ V electrons?

Answer : $\beta=\left(1+\left(E_{0} / p c\right)^{2}\right)^{-0.5}$

2. Measure the radius of the ISR tunnel on the CERN phone book map.

Answer : $150 \mathrm{~m}$.

3. If $43 \%$ of an electon synchrotron ring consists of bending magnetsand it matches the ISR tunnel - what is the radius of curvaturein these magnets?

Answer : 64.5

4. Assuming the maximum field is 0.5 Tesla what would be $B^{*}$ rhoand from it calculate the maximum energy?

Answer ; 26.68 T.m. ; 8 GeV kinetic

5. Suppose the quadrupoles have a bore diameter of $70 \mathrm{~mm}$.and the field at this radius is 0.42 Tesla. What is themaximum field gradient?

Answer $12 \mathrm{~T} / \mathrm{m}$

6. Suppose there are 144 quadrupoles - and thefocal length is about equal to $2 / 3$ of their spacing. What is thelength of each one?

Answer ; $0.51 \mathrm{~m}$.

7. Allowing $0.5 \mathrm{~m}$ at one end of the Bending magnet and $1.5 \mathrm{~m}$ at the other - what will be the length of the bending magnet? (Hint there are two bending magnets and two quads per FODO period)

Answer : $4.035 \mathrm{~m}$.

8. How many bending magnets are needed and how many full andempty half periods are there.

Answer 84 half perids will have bends and 60 will be empty. 
TUT6.XLS

\begin{tabular}{|c|c|c|c|c|}
\hline & A & $\mathbf{B}$ & $\mathbf{C}$ & D \\
\hline 1 & IMPROVED & ISR BEAUTY FACT & ORY FOR TUTORIAL OF & 2.Feb.91 \\
\hline \multicolumn{5}{|c|}{ (1) } \\
\hline 3 & & & Esub 0 & r sub. 0 \\
\hline 4 & & & 0.000511 & 0.00000 \\
\hline \multicolumn{5}{|l|}{5} \\
\hline 6 & & & \multicolumn{2}{|l|}{ Outined data s lnpu } \\
\hline \multicolumn{5}{|c|}{ 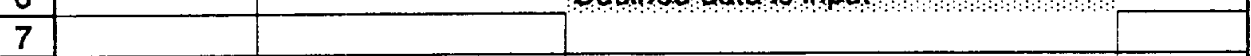 } \\
\hline 8 & & & Formulae & Data \\
\hline 9 & & Kinetic energy $(\mathrm{GeV})$ & 8 & 8 \\
\hline 10 & & Energy[GeV] & $=C 9+\$ C \$ 4$ & 8.00000 \\
\hline 11 & & gamma & $=\mathrm{C} 10 / \$ \mathrm{C} \$ 4$ & 15655.5 \\
\hline 12 & & beta & $=\operatorname{SQRT}\left(1-1 / \mathrm{C} 11^{\wedge} 2\right)$ & 0.99999 \\
\hline 13 & & Momentum[GeV/c] & $=\mathrm{C} 10^{*} \mathrm{C} 12$ & 8 \\
\hline 14 & & $\mathrm{~B}^{*} \mathrm{rho}(\mathrm{T} . \mathrm{m})$ & $=3.3356^{*} \mathrm{C} 13$ & 26.6848 \\
\hline 15 & & Ring radius $(m)$ & 150 & 150 \\
\hline 16 & & Fraction bend & 0.43 & 0.43 \\
\hline 17 & & Curvature $(m)$ & $=\mathrm{C} 15^{*} \mathrm{C} 16$ & 64.5 \\
\hline 18 & & Circumference $(m)$ & $=2^{*} 3.14159^{*} \mathrm{C} 15$ & 942.477 \\
\hline 19 & & Dipole field ( $T$ ) & 0.5 & 0.5 \\
\hline 20 & & $\mathrm{pc} \operatorname{Max}(\mathrm{GeV})$ & $=014 / 3.3356$ & 8 \\
\hline 21 & & Etot (max- $\mathrm{GeV})$ & $=\mathrm{C} 20 / \mathrm{C} 12$ & 8.00000 \\
\hline 22 & & Quad bore diam. $(\mathrm{m})$ & 0.07 & 0.07 \\
\hline 23 & & Pole tip field $(T)$ & 0.42 & 0.42 \\
\hline 24 & & Quad gradient & $=\mathrm{C} 23 /\left(0.5^{*} \mathrm{C} 22\right)$ & 12 \\
\hline 25 & & Normalised grad (k) & $=\mathrm{C} 24 / \mathrm{C} 14$ & 0.44969 \\
\hline 26 & & Number of quads & 144 & 144 \\
\hline 27 & & Spacing $(\mathrm{m})$ & $=\mathrm{C} 18 / \mathrm{C} 26$ & $6.54497 \mathrm{~s}$ \\
\hline 28 & & Focal length $(\mathrm{m})$ & $=2^{*} \mathrm{C} 27 / 3$ & 4.363319 \\
\hline 29 & & Quad length $(m)$ & $=1 / \mathrm{C} 28 / \mathrm{C} 25$ & 0.50964 \\
\hline 30 & & Short straight $(\mathrm{m})$ & 0.5 & 0.5 \\
\hline 31 & & Longer straight $(\mathrm{m})$ & 1.5 & 1.5 \\
\hline 32 & & Bending length & $=\mathrm{C} 27-\mathrm{C} 29-\mathrm{C} 30-\mathrm{C} 31$ & 4.03533 \\
\hline 33 & & Number dipoles & $=2^{*} 3.14159365^{*}\left(\mathrm{C}_{14} / \mathrm{C}_{19}\right) / \mathrm{C} 32$ & 83.0986 \\
\hline 34 & & Number half periods & $=\mathrm{C} 26$ & 144 \\
\hline 35 & & Filled/empty & $=\mathrm{C} 33 / \mathrm{C} 34$ & 0.57707 \\
\hline 36 & & & & \\
\hline 37 & & & & \\
\hline 38 & & Cos mu & $=1-\left(C 27^{\wedge} 2 /\left(2^{*} C 28^{\wedge} 2\right)\right)$ & -0.125 \\
\hline 39 & & Mu(rad) & $=A \cos (\mathrm{C} 38)$ & 1.69612 \\
\hline 40 & Ans $6 b$ & Mu(deg) & $=360^{\circ} \mathrm{C} 39 / 2 / 3.142$ & 97.1681 \\
\hline 41 & & $\mathrm{~m} 12$ & $=2^{*} \mathrm{C} 27^{*}\left(1+\mathrm{C} 27 /\left(2^{*} \mathrm{C} 28\right)\right)$ & 22.9074 \\
\hline 42 & Ans $6 c$ & Beta max & $=\mathrm{C} 41 /(\operatorname{SIN}(\mathrm{C} 39))$ & 23.0885 \\
\hline 43 & & $\mathrm{~m} 12$ & $=2^{*} \mathrm{C} 27^{*}\left(1-\mathrm{C} 27 /\left(2^{*} \mathrm{C} 28\right)\right)$ & 3.27248 \\
\hline 44 & Ans $6 d$ & Beta max & $=C 43 / \operatorname{SIN}(C 39)$ & 3.29835 \\
\hline 45 & & & & \\
\hline
\end{tabular}


